\title{
Synthesis and structure-activity relationships of novel lincomycin derivatives. Part 4: synthesis of novel lincomycin analogs modified at the 6- and 7-positions and their potent antibacterial activities
}

\author{
Yoshinari Wakiyama, Ko Kumura, Eijiro Umemura, Kazutaka Ueda, Takashi Watanabe, Keiko Yamada, \\ Takafumi Okutomi and Keiichi Ajito
}

To modify lincomycin (LCM) at the C-6 and the C-7 positions, we firstly prepared various substituted proline intermediates (7, 11-15 and 17). These proline intermediates were coupled with methyl 1-thio- $\alpha$-lincosamide and tetrakis- 0 -trimethylsilylation followed by selective deprotection of the TMS group at the 7-position gave a wide variety of key intermediates (23-27, 47 and 50). Then, we synthesized a variety of novel LCM analogs modified at the 7-position in application of the Mitsunobu reaction, an $\mathrm{S}_{\mathrm{N}} 2$ reaction, and a Pd-catalyzed cross-coupling reaction. Compounds 34 and 35 ( $1^{\prime}-\mathrm{NH}$ derivatives) exhibited enhanced antibacterial activities against resistant pathogens with erm gene compared with the corresponding $\mathbf{1}^{\prime}-\mathrm{N}$-methyl derivatives (3 and 37). On the basis of reported SAR, we modified the 4'-position of LCM derivatives possessing a 5-(2-nitrophenyl)-1,3,4thiadiazol-2-yl group at the C-7 position. Compound 56 showed significantly potent antibacterial activities against $S$. pneumoniae and $S$. pyogenes with erm gene, and its activities against $S$. pneumoniae with erm gene were improved compared with those of 34 and 57. Although we synthesized novel analogs by transformation of a C-7 substituent focusing on the $1^{\prime}$-demethyl framework to prepare very potent analogs 73 and 75 , it was impossible to generate novel derivatives exhibiting stronger antibacterial activities against $\boldsymbol{S}$. pneumoniae with erm gene compared with 56.

The Journal of Antibiotics (2017) 70, 888-906; doi:10.1038/ja.2017.54; published online 31 May 2017

\section{INTRODUCTION}

Macrolide antibiotics have been used in the clinical site for bacterial respiratory infections so far. Recently, macrolide-resistant bacteria with erm gene have markedly increased. ${ }^{1-3}$ Clarithromycin (CAM) ${ }^{4}$ and azithromycin ${ }^{5,6}$ are not effective enough against resistant pathogens such as Streptococcus pneumoniae and Streptococcus pyogenes with erm gene (Figure 1 and Table 1). Telithromycin (TEL) ${ }^{7}$ is effective enough against $S$. pneumoniae with erm gene, but has the potential to cause a serious liver damage ${ }^{8,9}$ and loss of consciousness. ${ }^{10,11}$ Thus, TEL has scarcely been used in Japan. Novel azalides reported by Miura et al. ${ }^{12,13}$ are also effective against resistant pathogens, but these analogs are still under research process and have not been developed yet. Currently available oral antibiotics are not effective enough against resistant bacteria with erm and mef genes causing respiratory infections and have some problems in safety or taste in clinical site.

Lincomycin $(\mathrm{LCM})^{14-17}$ and clindamycin $(\mathrm{CLDM})^{18}$ are effective against pathogens with mef gene in clinical isolates, but they are not effective against resistant pathogens with erm gene (Figure 1 and Table 1). As an overview, CLDM exhibits the following positive characters: (1) availability in per os and intravenous administrations (switch therapy is possible), (2) good distributions to tissue and cells,
(3) suppression ${ }^{19}$ of toxin production by Streptococcal strains and (4) expected reasonable production cost of LCM/CLDM derivatives. Thus, LCM derivatives might be more clinically valuable than macrolide antibiotics, if they are effective against pathogens with erm gene.

Chemical modifications at the C-7 position of LCM were achieved by several research groups. ${ }^{17,18,20-32}$ None of them, however, showed effective antibacterial activities against resistant bacteria with erm gene. On the other hand, we reported 7(S)-thiolincomycin analogs exhibiting antibacterial activities against resistant pathogens with $\mathrm{erm}$ gene as the first example ${ }^{33-36}$ as far as we know. We recently reported $7(S)$-thiolincomycin analogs, such as compounds $\mathbf{1 - 3}$ (Table 1), as the first-generation derivatives in our research. ${ }^{37}$ Those compounds possessed weak antibacterial activities against resistant $S$. pneumoniae with erm and mef genes, but compound $\mathbf{3}$ exhibited clearly improved activities compared with clarithromycin, azithromycin, LCM and CLDM as shown in Table 1. Recently, we also reported 7-S-substituted novel LCM derivatives, ${ }^{38-41}$ and some of them showed stronger activities than compound 3 did.

Chemical modification of a proline moiety at the C-6 position of LCM and CLDM was performed by several research groups. ${ }^{17,24,42-48}$

Pharmaceutical Research Center, Meiji Seika Pharma, Yokohama, Japan

Correspondence: Dr K Ajito, Pharmaceutical Research Center, Meiji Seika Pharma, 760 Morooka-cho, Kohoku-ku, Yokohama 222-8567, Japan.

E-mail: keiichi.ajito@meiji.com

Received 18 January 2017; accepted 20 March 2017; published online 31 May 2017 


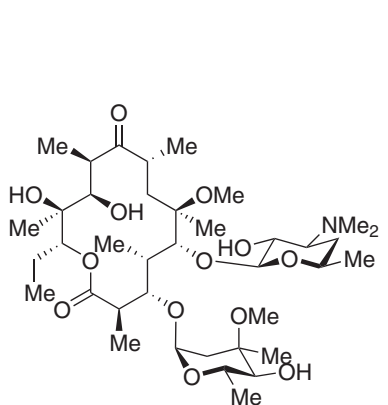

Clarithromycin (CAM)

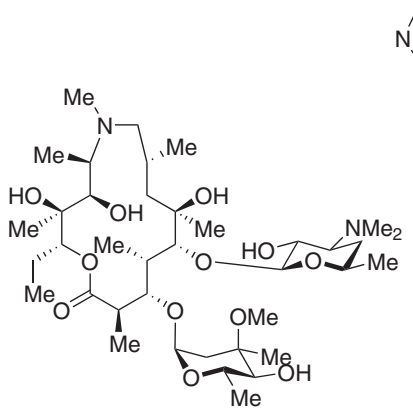

Azithromycin (AZM)

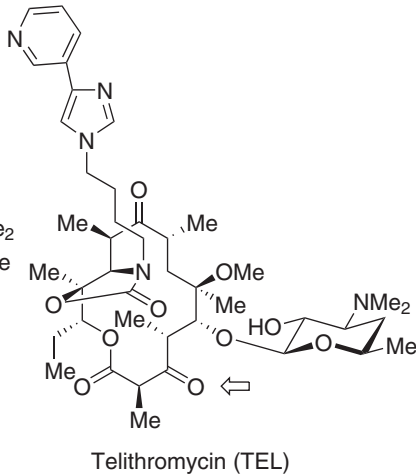

Me

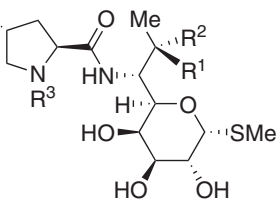

Lincomycin (LCM): $\mathrm{R}^{1}=\mathrm{OH}, \mathrm{R}^{2}=\mathrm{H}, \mathrm{R}^{3}=\mathrm{Me}$ 1'-Demethyllincomycin: $\mathrm{R}^{1}=\mathrm{OH}, \mathrm{R}^{2}=\mathrm{H}, \mathrm{R}^{3}=\mathrm{H}$ Clindamycin (CLDM): $\mathrm{R}^{1}=\mathrm{H}, \mathrm{R}^{2}=\mathrm{Cl}, \mathrm{R}^{3}=\mathrm{Me}$ $1^{\prime}$-Demethylclindamycin: $\mathrm{R}^{1}=\mathrm{H}, \mathrm{R}^{2}=\mathrm{Cl}, \mathrm{R}^{3}=\mathrm{H}$<smiles>[R]C1C[C@H](C(=O)N[C@H](C(C)O)[C@H]2O[C@H](C)[C@@H](O)[C@H](O)[C@H]2O)CN1</smiles>

4'-Substituted lincomycins: $\mathrm{R}=\mathrm{H}$, Me to ${ }^{n_{\text {octyl }}}$ 4'-Depropyl-4'-ethoxylincomycin: $\mathrm{R}=$ ethoxy 4'-Depropyl-4'-ethoxycarbonylmethyllincomycin: $\mathrm{R}=$ ethoxycarbonylmethyl 4'-Depropyl-4'- $p$-toluenesulfonyloxylincomycin: $\mathrm{R}=p$-toluensulfonyloxy
$\mathrm{R}^{2}, 4$

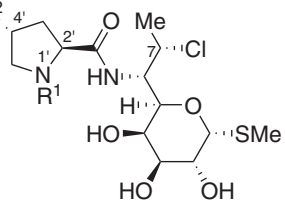

4'-Depropyl-4'- ${ }^{n}$ pentylclindamycin:

$$
\mathrm{R}^{1}=\mathrm{Me}, \mathrm{R}^{2}={ }^{n} \text { pentyl }
$$

1'-Demethyl-4'-depropyl-4'-- ${ }^{n}$ pentylclindamycin: $\mathrm{R}^{1}=\mathrm{H}, \mathrm{R}^{2}={ }^{n}$ pentyl
$\mathrm{Me}$<smiles>CCCCC[C@H]1C[NH2+]C(C(=O)N[C@H](C(C)C)[C@H]2O[C@H](C)[C@@H](O)[C@H](O)[C@H]2O)C1</smiles>

VIC105403: $\mathrm{R}^{1}=\mathrm{H}$

VIC105404: $R^{1}=2$-hydroxyethyl

VIC105405: $\mathrm{R}^{1}=2-(R)$-hydroxypropyl

VIC105446: $R^{1}=2-(S)$-hydroxypropyl

Figure 1 Chemical structures of clarithromycin, azithromycin, telithromycin, lincomycin, clindamycin and a variety of reported analogs of lincomycin or clindamycin.

Table 1 Anti-bacterial activities (MIC, $\mu \mathrm{g} \mathrm{ml}^{-1}$ ) of CAM, AZM, LCM, CLDM, and previously reported LCM derivatives (1-3)

$$
\text { Me_" }
$$

Test organism

Streptococcus pneumoniae DP1 type

S. pneumoniae-2

S. pneumoniae-3

S. pneumoniae-4

S. pneumoniae-5

S. pneumoniae-6

S. pneumoniae-7

S. pneumoniae-8

S. pneumoniae- 9

Streptococcus pyogenes Cook

S. pyogenes-2

S. pyogenes-3

Haemophilus influenzae

H. influenzae-2

$H$. influenzae-3

$H$. influenzae-4

Characteristics
Susceptible
Susceptible
Susceptible
ermAM methyl
ermAM methyl
ermAM methyl
ermAM methyla
ermAM methyla
mefE efflux
Susceptible
ermAM methyla
mefE efflux
Susceptible
Susceptible
Susceptible
$\triangle a c r$

Abbreviations: AZM, azithromycin; CAM, clarithromycin. CLDM, aAll strains except standard organisms were clinically isolated.

${ }^{b}(c)$, constitutive; (i), inducible.

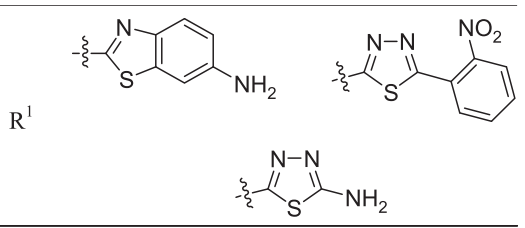

\begin{tabular}{ccccccc} 
CAM & AZM & LCM & CLDM & $\mathbf{1}$ & $\mathbf{2}$ & $\mathbf{3}$ \\
\hline 0.03 & 0.06 & 1 & 0.06 & 0.06 & 0.03 & 0.015 \\
0.03 & 0.03 & 1 & 0.12 & 0.06 & 0.06 & 0.015 \\
0.015 & 0.03 & 0.25 & 0.06 & 0.03 & 0.06 & 0.03 \\
$>128$ & $>128$ & $>128$ & $>128$ & 16 & 16 & 4 \\
$>128$ & $>128$ & $>128$ & $>128$ & 64 & 64 & 8 \\
$>128$ & $>128$ & $>128$ & $>128$ & 64 & 128 & 16 \\
$>128$ & $>128$ & 128 & 128 & 16 & 16 & 2 \\
$>128$ & $>128$ & 128 & 128 & 8 & 16 & 2 \\
0.5 & 0.5 & 1 & 0.12 & 0.06 & 0.03 & 0.015 \\
& & & & & & \\
0.015 & 0.06 & 0.12 & 0.06 & 0.03 & 0.03 & 0.03 \\
$>128$ & $>128$ & $>128$ & 128 & 8 & 8 & 2 \\
8 & 8 & 0.25 & 0.12 & 0.06 & 0.06 & 0.03 \\
& & & & & & \\
2 & 0.25 & 8 & 16 & 8 & 8 & 16 \\
4 & 1 & 16 & 8 & 4 & 4 & 8 \\
8 & 2 & 16 & 16 & 32 & 8 & 16 \\
0.5 & 0.5 & 4 & 1 & 0.25 & 0.25 & 0.25 \\
\hline
\end{tabular}


$R^{1}$
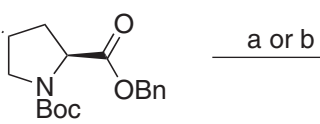

4: $\mathrm{R}^{1}=$ allyl

5: $R^{1}=\overbrace{M e}$

6: $\mathrm{R}^{1}=\mathrm{Me}$

c

4<smiles>CCCCO</smiles><smiles>C1CCC(C2CCCCC2)CC1</smiles><smiles>O=C(OCc1ccccc1)[C@@H]1CCCN1</smiles>

8

$R^{2}=T B S$

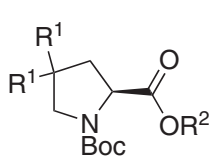

16: $R^{1}=$ allyl; $R^{2}=B n \square$
17: $R^{1}={ }^{n} \operatorname{Pr} ; R^{2}=H$

11: $R^{2}=$ TBS

12: $R^{2}=M e$

Scheme 1 Synthesis of proline derivatives. Conditions: (a) $\mathrm{H}_{2}, \mathrm{Pd} / \mathrm{C}, \mathrm{MeOH}$, r.t., $0.5-4.5 \mathrm{~h}$; (b) $1 \mathrm{~N} \mathrm{NaOH}, \mathrm{MeOH}$, r.t., $22 \mathrm{~h}$; (c) (1) 9-BBN, THF, $50{ }^{\circ} \mathrm{C}$, $1 \mathrm{~h}$, (2) $1 \mathrm{~N} \mathrm{NaOH}, 35 \% \mathrm{H}_{2} \mathrm{O}_{2}, 0^{\circ} \mathrm{C}, 2 \mathrm{~h}$; (d) $\mathrm{TBSCl}$, imidazole, DMF, r.t., $30 \mathrm{~min}$; (e) Mel, NaH, DMF, r.t., $30 \mathrm{~min}$. 9-BBN, 9-borabicyclo[3.3.1] nonane; DMF, dimethylformamide; Mel, methyl iodide; TBSCl, tertbutyldimethylsilyl chloride; r.t., room temperature; THF, tetrahydrofuran.

This portion has the following features: (1) Regarding configuration between the $2^{\prime}$ - and $4^{\prime}$-positions of a proline ring, an analog with trans configuration exhibited more potent antibacterial activity compared with one with cis configuration. (2) 4'-Depropyl-3'-propyllincomycin has not been synthesized, but 4'-depropyl-5'-propyllincomycin has already been synthesized. Its antibacterial activity was weaker than that of LCM. (3) 1'-Demethylclindamycin (Figure 1) was two times as active in vitro against Sarcina lutea as CLDM, but 1'-demethyllincomycin was about one-twentieth as active as LCM (relative potency against $S$. lutea: 1'-demethylclindamycin $>$ CLDM $>$ LCM $>1^{\prime}$ demethyllincomycin $=8>4>1>0.05) .{ }^{21,42-43} \quad 1^{\prime}$-Demethyl $-1^{\prime}-N-$ ethyllincomycin possessed the same activity as LCM. (4) Regarding SAR of a chain length $\left(\mathrm{H}, \mathrm{Me}\right.$ to $n$-octyl) at the $4^{\prime}$-position of the proline moiety for LCM (Figure 1), the in vitro antibacterial activities were enhanced until reaching a maximum at the hexyl analog. ${ }^{21,44}$ A similar in vivo activity was indicated, but maximum effect was exhibited by an $n$-pentyl group. Furthermore, alternative in vitro SAR were observed by changing a chain length (Figure 1) for a $4^{\prime}$-alkylsubstituent of CLDM and a $4^{\prime}$-alkyl-substituent of $1^{\prime}$-demethylclindamycin (relative potency: $4^{\prime}$-depropyl- $4^{\prime}-n$-pentylclindamycin > CLDM, $1^{\prime}$-demethyl-4'-depropyl- $4^{\prime}-n$-pentylclindamycin $>1^{\prime}$-demethylclindamycin). ${ }^{21}$ However, antibacterial activities of those compounds against resistant pathogens with erm gene were not disclosed. (5) $4^{\prime}$-Depropyl-4'-ethoxylincomycin had only $\sim 2 \%$ antibacterial activities of LCM, and 4'-depropyl-4'-ethoxycarbonylmethyllincomycin and $4^{\prime}$-depropyl-4'-p-toluenesulfonyloxylincomycin were essentially inactive in antibacterial testing. ${ }^{17,21,45}$

On the other hand, Vicuron, which was consolidated by Pfizer, reported the following compounds. VIC 105403 (Figure 1), ${ }^{24}$ possessing $1^{\prime}$-demethyl, $4^{\prime}$-n-pentyl and 7-methyl group, had potent activities compared with CLDM. VIC $105404,{ }^{24}$ possessing $1^{\prime}$-N-(2-hydroxyethyl), 4'-n-pentyl and 7-methyl group, was already reported to have potent antibacterial activities as VIC 105403. However, those did not show antibacterial activities against resistant pathogens with erm gene. Furthermore, VIC 105405 and VIC $105446,{ }^{24}$ having a branched side chain at the $1^{\prime}$-position, showed less antibacterial activities than CLDM. Other VIC compounds possessing a branched side chain, a 3-(pyridin-4-yl)propyl or an $n$-butylthio group at the $4^{\prime}$-position, were already synthesized, but their antibacterial activities were not disclosed in their patent. ${ }^{46}$

According to X-ray crystallographic analysis by Yonath et al., ${ }^{47}$ we hypothesized that CLDM had enough three-dimensional space around the 1 '-position and it might be able to enhance the antibacterial activities by filling the corresponding space with other functional groups except the Me group. On the basis of the above hypothesis, we designed and synthesized novel $7(S)$-substituted LCM derivatives modified at the $1^{\prime}$ - and/or $4^{\prime}$-position(s) at the proline moiety. Anti-bacterial activities of these novel compounds are also disclosed in this report.

\section{RESULTS AND DISCUSSION}

Synthesis of proline derivatives

Synthesis of proline derivatives is shown in Scheme 1. Synthetic route for modification of the proline moiety at the 4 '-position was already reported by Pedregal et al. ${ }^{48,49}$ We firstly prepared starting materials (4-6 and 16), and compound 8 was synthesized by hydroboration of 4 followed by treatment with tert-butyldimethylsilyl chloride (TBSCl) or methyl iodide under the basic condition to give compounds $\mathbf{9}$ and $\mathbf{1 0}$. The compounds 4-6, 9, 10 and 16 were reduced with $\mathrm{H}_{2}$ in the presence of $\mathrm{Pd} / \mathrm{C}$ to give the corresponding carboxylic acids $(7,11-14$ and 17$)$, respectively. Compound 15 was prepared by hydrolysis of $6 .{ }^{1} \mathrm{H}$ NMR spectra of compounds 7, 8, 10, 13-15 and 17 were observed as two sets of signals because of a rotamer by the tert-butoxycarbonyl (Boc) group.

Synthesis of key intermediates 23-27 and transformation of 23 and 36 Synthesis of key intermediates 23-27 and transformations of 23 and 36 are shown in Scheme 2. Compounds 18-22 were synthesized by condensation of compounds 7 and 12-15 with methyl 1-thio- $\alpha-$ lincosamide (MTL), respectively. Tetra-O-trimethylsiliylation of all hydroxyl groups and successive regioselective deprotection of the TMS group at the 7-position gave key intermediates (23-27). Furthermore, novel LCM derivatives 32-35 were synthesized via two or three steps from compound 23 by the Mitsunobu reaction at the 7-position and deprotection of the TMS groups and the Boc group at the 1'-position. On the other hand, $1^{\prime}$ $N$-methyl analog 37 was prepared from 36 following the similar procedure as the above. ${ }^{1} \mathrm{H}$ NMR spectra of compounds $\mathbf{1 8 - 2 0}$ and 23 were observed as two sets of signals because of a rotamer by the Boc group and compounds 25-27 showed broad peaks in ${ }^{1} \mathrm{H}$ NMR spectra. Compounds 32-35 whose Boc groups were deprotected, however, showed a single set of signals and sharp peaks in ${ }^{1} \mathrm{H}$ NMR spectra.

Synthesis of $1^{\prime}-N$-modified novel LCM derivatives possessing a substituted 1,3,4-thiadiazol-2-ylthio group at the 7-position Synthesis of $1^{\prime}$ - $N$-modified novel LCM derivatives possessing the substituted 1,3,4-thiadiazol-2-ylthio group at the 7-position is shown in Scheme 3. Desired 1'-N-modified derivatives 38, 39, 41 and 45 were prepared from 34 or 35 by reductive aminoalkylation. Consequently, the TBS groups of $\mathbf{3 9}$ and $\mathbf{4 1}$ were removed by TBAF to give compounds 40 and 42 , respectively. Compound 43 was 

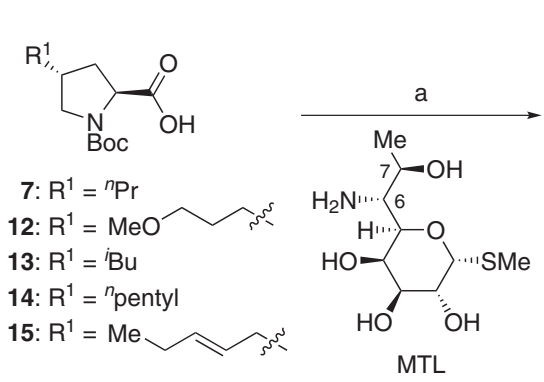

$\mathrm{R}^{1}, 4^{\prime}$

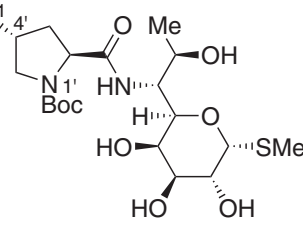

18: $\mathrm{R}^{1}={ }^{n} \mathrm{Pr}$

19: $\mathrm{R}^{1}=\mathrm{MeO}$

20: $R^{1}={ }^{i} B u$

21: $R^{1}={ }^{n}$ pentyl

22: $R^{1}=\mathrm{Me}$

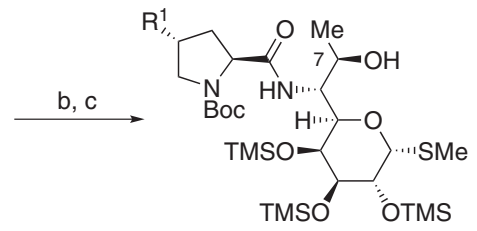

23: $\mathrm{R}^{1}={ }^{n} \mathrm{Pr}$

24: $\mathrm{R}^{1}=\mathrm{MeO}$ 는

25: $R^{1}=i B u$

26: $\mathrm{R}^{1}={ }^{n}$ pentyl

27: $\mathrm{R}^{1}=\mathrm{Me}$

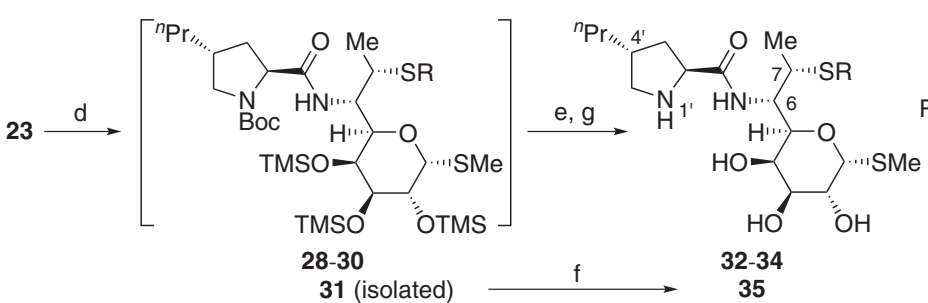

31 (isolated)
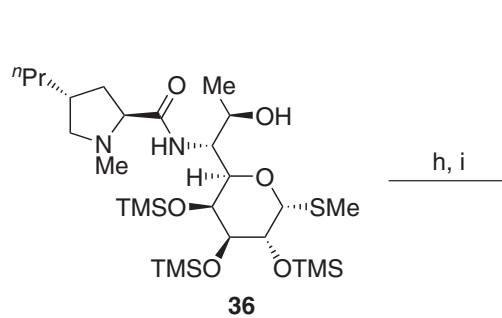<smiles>CNc1scnc1-c1nnc(S[C@@H](C)[C@H](NC(=O)C2C[C@@H](C(C)C)CN2)[C@H]2O[C@H](C)[C@@H](O)[C@H](O)[C@H]2O)s1</smiles>

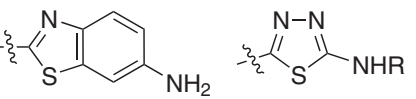

R: $28,32 \quad 29: \mathrm{R}=\mathrm{Boc} ; 33: \mathrm{R}=\mathrm{H}$<smiles>Cc1nnc(-c2ccccc2[N+](=O)[O-])s1</smiles>

30,34

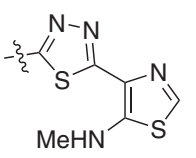

31,35

Scheme 2 Synthesis of key intermediates 23-27 and transformation of 23 and 36. Conditions: (a) MTL, DCC, HOBt, DMF, r.t., 3-23 h; (b) TMSCl, HMDS, Py, r.t., 1-7 h; (c) $6 \mathrm{~N} \mathrm{AcOH}, \mathrm{MeOH}$, r.t., 0.5-11 h; (d) DEAD, PPh 3 , HSR (the corresponding heteroaromatic thiols), THF, $0{ }^{\circ} \mathrm{C}$ to r.t., 3-18 h; (e) $1 \mathrm{~N} \mathrm{HCl}$, $\mathrm{MeOH}$, r.t., 0.5-2.5 h; (f) $4 \mathrm{~N} \mathrm{HCl-EtOAc,} \mathrm{MeOH}, 0{ }^{\circ} \mathrm{C}$ to r.t., 4.5 h; (g) $4 \mathrm{~N} \mathrm{HCl-EtOAc}$, MeOH, r.t., 2-2.5 h; (h) DEAD, PPh yl)-1,3,4-thiadiazole-2-thiol, THF, r.t., $2 \mathrm{~h}$, (i) $1 \mathrm{~N} \mathrm{HCl}, \mathrm{MeOH}$, r.t., $1 \mathrm{~h}$. DCC, dicyclohexylcarbodiimide; DEAD, diethyl azodicarboxylate; DMF, dimethylformamide; HMDS, hexamethyldisilazane; HOBt, 1-hydroxybenzotriazol; MTL, methyl 1-thio- $\alpha$-lincosamide; PPh 3 , triphenylphosphine; Py, pyridine; THF, tetrahydrofuran; TBSCl, tert-butyldimethylsilyl chloride; r.t., room temperature.

synthesized in application of $(R)$-2-methyloxirane under the basic condition from 35. Compound 44 was also prepared by acetic anhydride from 35 .

Synthesis of novel LCM derivatives 48 and 53 possessing a germinal bis-propyl moiety or a 3-(dimethylamino)propyl group at the 4 '-position

Synthesis of novel LCM derivatives $\mathbf{4 8}$ and $\mathbf{5 3}$ possessing a germinal bis-propyl moiety and a 3-(dimethylamino)propyl group, respectively, at the 4 -position, and having the 5-(2-nitrophenyl)-1,3,4-thiadiazol2-ylthio group at the 7-position, is shown in Scheme 4. Key intermediates $\mathbf{4 7}$ and $\mathbf{5 0}$ were, respectively, synthesized in the application of condensation, tetrakis-O-trimethylsilylation, and selective deprotection with the similar procedure to compound 23. Later, a 7 -S-substituent was introduced to 47 by the Mitsunobu reaction, and deprotection gave a desired derivative 48. Compound $\mathbf{5 0}$ was transformed to 51 by the Mitsunobu reaction and desilylation. Then, compound $\mathbf{5 1}$ was reacted with sodium azide, and an azide group was reduced to an amino group with triphenylphosphine-water. The afforded amino group in $\mathbf{5 2}$ was applied with reductive aminoalkylation followed by deprotection of the Boc group under the acidic condition with trifluoroacetic acid (TFA) to give the desired novel derivative 53. ${ }^{1} \mathrm{H}$ NMR spectra of compounds $\mathbf{4 7}$ and $\mathbf{5 0}$ were also observed as broad peaks by the influence of a Boc group. Although the final product $\mathbf{5 3}$ could be purified as a single molecule, both intermediates $\mathbf{5 1}$ and $\mathbf{5 2}$ partially included impurity that was not removed by purification steps.

Synthesis of novel LCM derivatives with a variety of $4^{\prime}$-substituents possessing the substituted 1,3,4-thiadiazol-2-yl-thio group at the 7-position

Synthesis of novel LCM derivatives having a 3-methoxypropyl, an $i$-butyl or an $n$-pentyl group at the $4^{\prime}$-position, whose 7 -position was the substituted 1,3,4-thiadiazol-2-yl-thio group, is shown in Scheme 5. Compounds 54-56 and 58 were prepared from key intermediates $\mathbf{2 4}-\mathbf{2 6}$ by the similar procedure to compound 48 . Reductive aminoalkylation of $\mathbf{5 6}$ and $\mathbf{5 8}$ afforded the desired compounds 57 and 59, respectively.

Synthesis of proline-modified novel LCM derivatives possessing a (4-morpholinocarbonyl)phenylthio group at the 7-position

Synthesis of proline-modified novel LCM derivatives possessing a (4-morpholinocarbonyl)phenylthio group at the 7-position is shown in Scheme 6. Because LCM derivatives possessing a (4-morpholinocarbonyl)phenylthio group at the 7-position exhibited improved antibacterial activities, we designed compounds 67-69. We already reported the methanesulfonyl (Ms) route ${ }^{37,38}$ to introduce a phenyl group via sulfur atom at the 7-position. The Ms route was applied to compounds 23 and 27 to give intermediates 60 and 61, respectively. ${ }^{1} \mathrm{H}$ NMR spectra of compounds $\mathbf{6 0 - 6 2}$ were observed as two sets of 


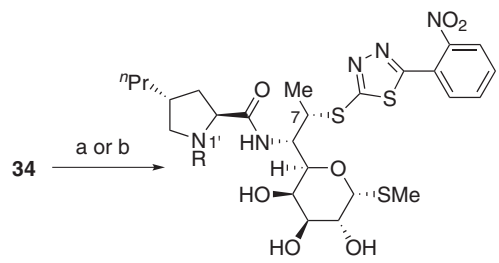

38: $\mathrm{R}={ }^{i} \mathrm{Pr}$

39: $\mathrm{R}=\mathrm{CH}_{2} \mathrm{CH}_{2} \mathrm{OTBS}$ 40: $\mathrm{R}=\mathrm{CH}_{2} \mathrm{CH}_{2} \mathrm{OH}$

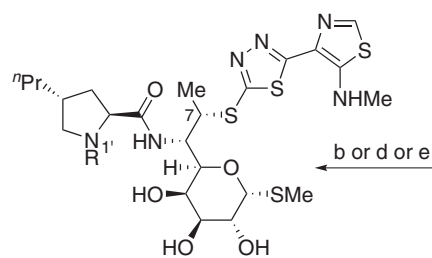

41: $\mathrm{R}=\mathrm{CH}_{2} \mathrm{CH}_{2} \mathrm{OTBS} \longrightarrow \mathrm{c}$

42: $\mathrm{R}=\mathrm{CH}_{2} \mathrm{CH}_{2} \mathrm{OH}$

43: $\mathrm{R}=(R)$-propan-2-ol

44: $R=A c$

Scheme 3 Synthesis of $1^{\prime}$ - $N$-modified novel LCM derivatives possessing the substituted 1,3,4-thiadiazol-2-ylthio group at the 7-position. Conditions: (a) acetone, $\mathrm{NaBH}(\mathrm{OAc})_{3}, \mathrm{AcOH}, \mathrm{ClCH}_{2} \mathrm{CH}_{2} \mathrm{Cl}, 0{ }^{\circ} \mathrm{C}$ to r.t., $15 \mathrm{~h}$; (b) 2-(tert-butyldimethylsilyloxy)acetaldehyde, $\mathrm{NaBH}(\mathrm{OAc})_{3}, \mathrm{AcOH}, \mathrm{ClCH}_{2} \mathrm{CH}{ }_{2} \mathrm{Cl}, 0{ }^{\circ} \mathrm{C}$ to r.t., $15 \mathrm{~h}$ for 39 , r.t., $15 \mathrm{~h}$ for 41 ; (c) TBAF, THF, $0{ }^{\circ} \mathrm{C}$ to r.t., $15 \mathrm{~h}$ for $40,5 \mathrm{~h}$ for 42 ; (d) (R)-2-methyloxirane, $i-\mathrm{Pr}_{2} \mathrm{NEt}, \mathrm{MeOH}^{\circ} 0{ }^{\circ} \mathrm{C}, 16 \mathrm{~h}$; (e) $\mathrm{Ac}_{2} \mathrm{O}, \mathrm{MeOH}, 0{ }^{\circ} \mathrm{C}, 1.5 \mathrm{~h}$; (f) 4-methylthiazole-5-carbaldehyde, $\mathrm{NaBH}(\mathrm{OAc})_{3}, \mathrm{AcOH}, \mathrm{MeOH}$, r.t., $15 \mathrm{~h}$. LCM, lincomycin; TBAF, tetra- $n$-butylammonium fluoride; THF, tetrahydrofuran; r.t., room temperature.

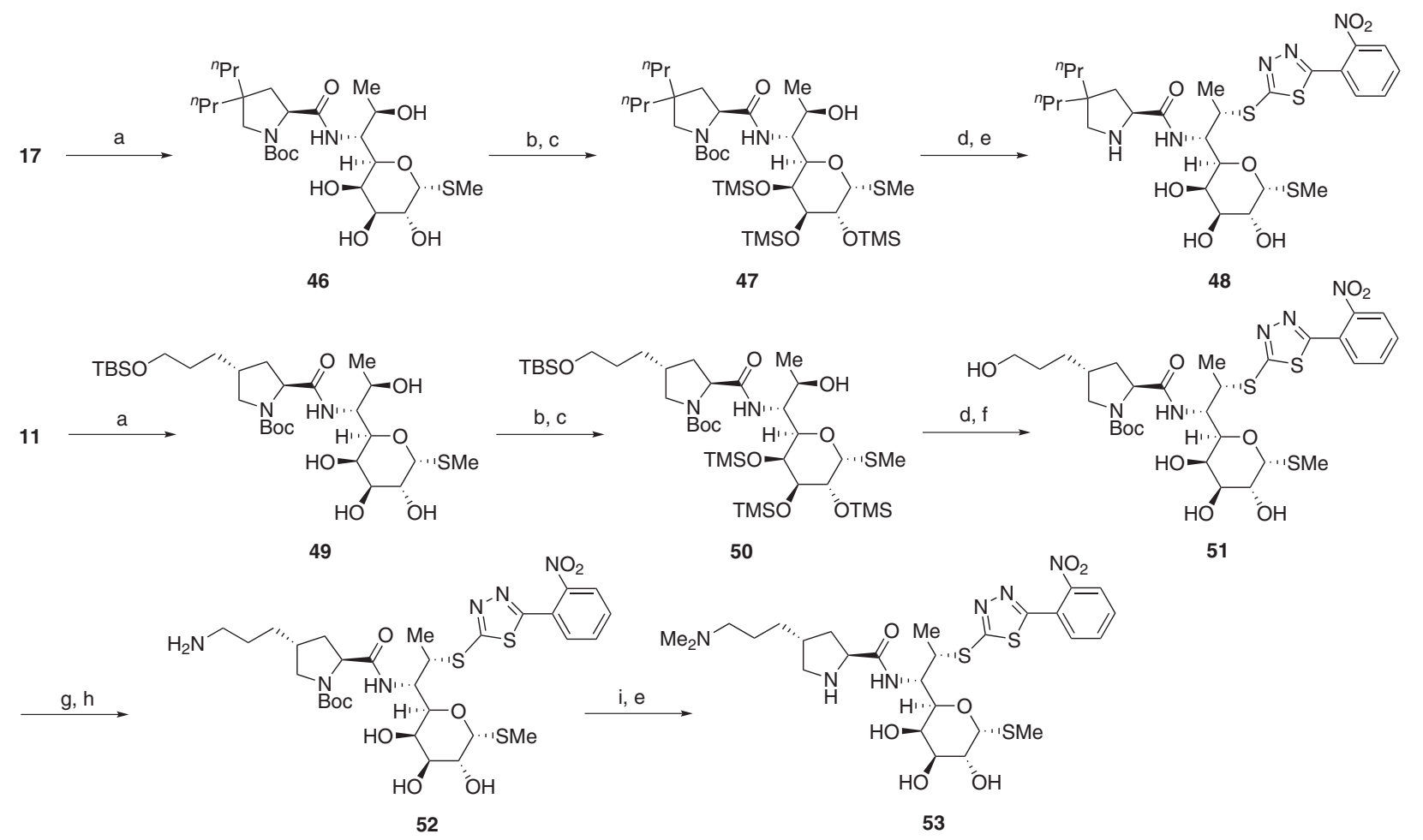

Scheme 4 Synthesis of 48 and 53. Conditions: (a) MTL, DCC, HOBt, DMF, r.t., 14 h; (b) TMSCl, HMDS, Py, r.t., $1 \mathrm{~h}$ from $46,20 \mathrm{~min}$ from 49 ; (c) $6 \mathrm{~N}$ $\mathrm{AcOH}, \mathrm{MeOH}$, r.t., 1-1.5 h; (d) 5-(2-nitrophenyl)-1,3,4-thiadiazole-2-thiol, DEAD, $\mathrm{PPh}_{3}$, THF, $0{ }^{\circ} \mathrm{C}$ to r.t., $6-10 \mathrm{~h}$; (e) $\mathrm{TFA}$, $0{ }^{\circ} \mathrm{C}$ to r.t., $30 \mathrm{~min}$; (f) TBAF, AcOH, THF, r.t., 5 h; (g) $\mathrm{NaN}_{3}, \mathrm{CBr}_{4}, \mathrm{PPh}_{3}, \mathrm{DMF}$, r.t. to $50^{\circ} \mathrm{C}, 2$ h; (h) $\mathrm{PPh}_{3}, \mathrm{H}_{2} \mathrm{O}$, THF, r.t. to $50{ }^{\circ} \mathrm{C}, 3 \mathrm{~h}$; (i) $\mathrm{HCHO}, \mathrm{NaBH}(\mathrm{OAc})_{3}, \mathrm{AcOH}$, $\mathrm{MeOH}$, r.t., 20 min. DCC, dicyclohexylcarbodiimide; DEAD, diethyl azodicarboxylate; DMF, dimethylformamide; HMDS, hexamethyldisilazane; HOBt, 1-hydroxybenzotriazol; MTL, methyl 1-thio- $\alpha$-lincosamide; $\mathrm{PPh}_{3}$, triphenylphosphine; Py, pyridine; TBAF, tetra-n-butylammonium fluoride; TFA, trifluoroacetic acid; THF, tetrahydrofuran; r.t., room temperature.

signals because of a rotamer by the Boc group. The desired analogs 67 and 68 were prepared from 60 and 62 , respectively, by (i) hydrolysis under the basic condition, (ii) condensation with morpholine and (iii) deprotection of the Boc group with TFA. Compound 69 was synthesized from 68 by reductive aminoalkylation with HCHO and $\mathrm{NaBH}(\mathrm{OAc})_{3}$ in an acidic condition by $\mathrm{AcOH}$.

\section{Synthesis of 73 and 75}

Synthesis of novel LCM derivatives 73 and 75 is shown in Scheme 7. We have already reported a $\mathrm{Pd}$-catalyzed cross-coupling route to introduce an aryl group at the 7-position of 7(S)-7-deoxy-7mercaptlincomycin. ${ }^{40} \mathrm{~A}$ key intermediate 71 was prepared via four steps from 23, and precursors 72 and 74 were synthesized by Pd-catalyzed cross-coupling reaction of $\mathbf{7 1} \mathbf{1}^{40,50}$ with the corresponding bromides. Deprotection of the Boc group afforded the desired analogs 73 and 75. ${ }^{1} \mathrm{H}$ NMR spectra of compounds 72 and 74 were observed as two sets of signals because of a rotamer by the Boc group, but both of the final compounds $\mathbf{7 3}$ and $\mathbf{7 5}$ showed a single set and sharp peaks in NMR spectra.

SAR analysis of 7-S-substituted $1^{\prime}-N H$ LCM derivatives (32-35) and $1^{\prime}-\mathrm{N}$-Me analog 37

Antibacterial activity of LCM was reduced by $\mathrm{l}^{\prime}$ - $\mathrm{N}$-demethylation, but that of CLDM was enhanced by $1^{\prime}-N$-demethylation. Thus, we were 
$\mathrm{R}^{1}$<smiles>COCCCC(C)(C)C</smiles>

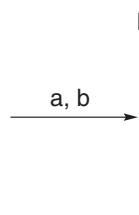<smiles>CCC1CNC(C(=O)N[C@H]([C@H](C)[SnH])[C@H]2O[C@H](C)[C@@H](O)[C@H](O)[C@H]2O)C1</smiles>

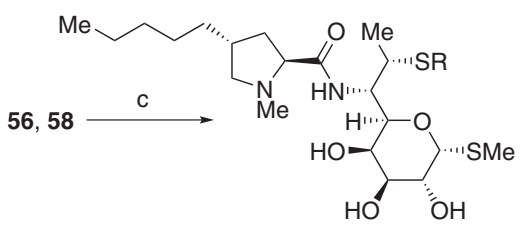

54: $R^{1}=\mathrm{MeO}$
55: $R^{1}={ }^{i} \mathrm{Bu}$
56: $\mathrm{R}^{1}={ }^{n}$ Pentyl

58: $\mathbf{R}^{1}={ }^{n}$ Pentyl<smiles>[2H][14c]1nnc(-c2ccccc2[N+](=O)[O-])s1</smiles><smiles>[R][Y]1nnc(-c2ncsc2NC)s1</smiles><smiles>O=[N+]([O-])c1ccccc1-c1nn[Y]([18F])s1</smiles>

59: $\mathrm{R}$<smiles>CNc1scnc1-c1nnc(I)s1</smiles>

Scheme 5 Synthesis of novel LCM derivatives with a variety of $4^{\prime}$-substituents possessing the substituted 1,3,4-thiadiazol-2-yl-thio group at the 7-position. Conditions: (a) DEAD, $\mathrm{PPh}_{3}, \mathrm{HSR}$, THF, $0{ }^{\circ} \mathrm{C}$ to r.t., 9.5-24 h; (b) TFA, $0{ }^{\circ} \mathrm{C}$ to r.t., 15-90 min; (c) $\mathrm{HCHO}, \mathrm{NaBH}(\mathrm{OAc})_{3}, \mathrm{AcOH}$, $\mathrm{MeOH}$, r.t., 1 h. DEAD, diethyl azodicarboxylate; LCM, lincomycin; TFA, trifluoroacetic acid; THF, tetrahydrofuran; r.t., room temperature.

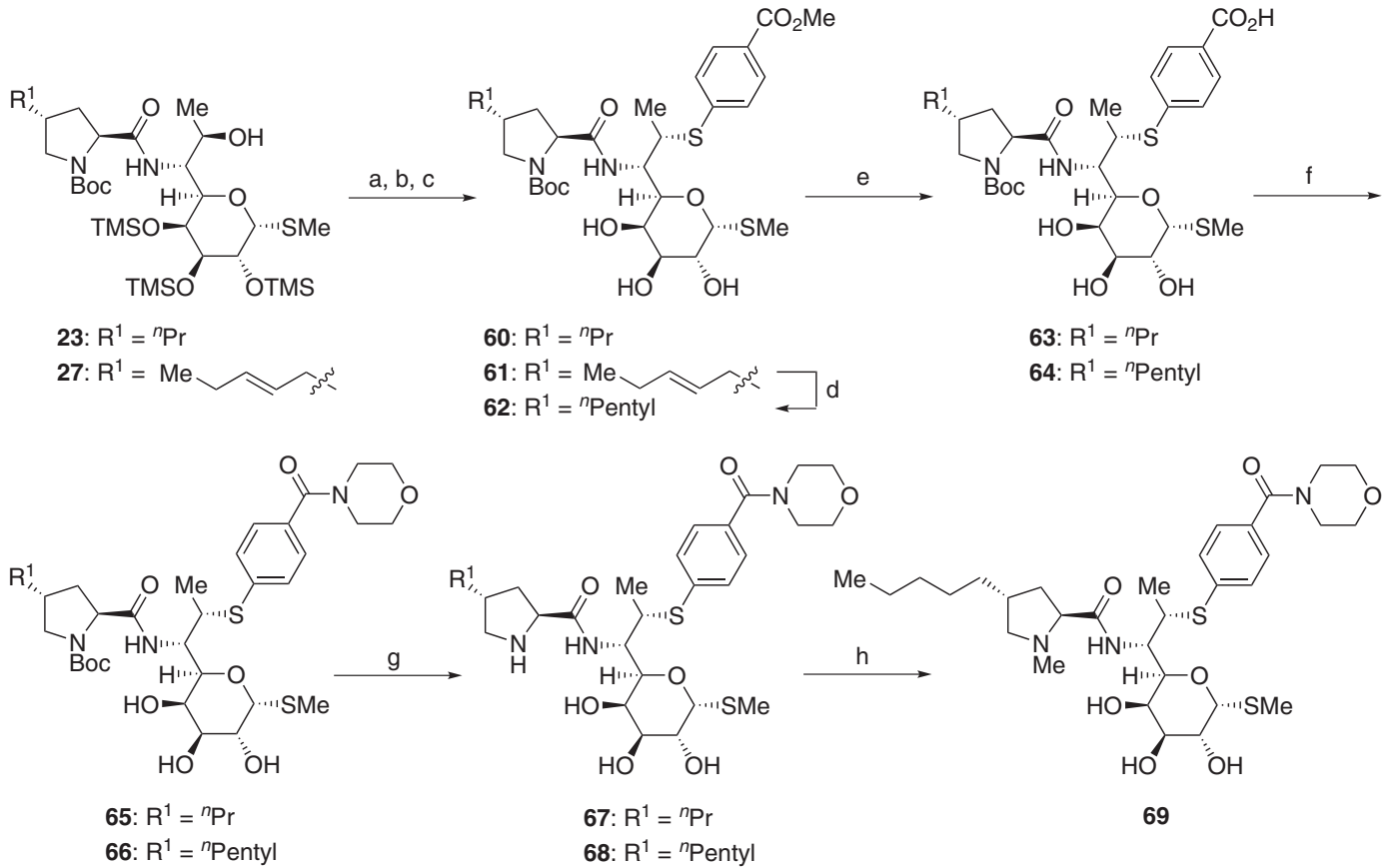

Scheme 6 Synthesis of proline-modified novel LCM derivatives possessing a (4-morpholinocarbonyl)phenylthio group at the 7-position. Conditions: (a) MsCl, $\mathrm{NEt}_{3}, \mathrm{CHCl}_{3}$, r.t., $30 \mathrm{~min}$; (b) methyl 4-mercaptobenzoate, $\mathrm{K}_{2} \mathrm{CO}_{3}$, DMF, 80-100 ${ }^{\circ} \mathrm{C}, 1-6$ h; (c) $1 \mathrm{~N} \mathrm{HCl}$, $\mathrm{MeOH}$, r.t., $20 \mathrm{~min}$; (d) $\mathrm{H}$, $\mathrm{Pd} / \mathrm{C}$, $\mathrm{MeOH}$, r.t., 15 h; (e) $1 \mathrm{~N} \mathrm{NaOH}, \mathrm{MeOH}$, r.t., 1-7 days; (f) morpholine, WSC, HOBt, DMF, r.t. 22-62 h, (g) TFA, -15 to $0{ }^{\circ} \mathrm{C}, 40 \mathrm{~min}$ and (h) $\mathrm{HCHO}, \mathrm{AcOH}$, $\mathrm{NaBH}(\mathrm{OAc})_{3}$, $\mathrm{MeOH}, 30$ min. DMF, dimethylformamide; HOBt, 1-hydroxybenzotriazol; LCM, lincomycin; r.t., room temperature; TFA, trifluoroacetic acid; WSC, watersoluble carbodiimide.

interested in the potency of $1^{\prime}$-N-demethyl products of $7(S)$-substituted LCM derivatives $\mathbf{1 - 3}$ as shown in Table 1 . Thus, we synthesized 1'-demethyl analogs of 1-3 (32-34) and compounds 35 and 37 possessing an alternative 7 -substituent. Antibacterial activities of those are shown in Table 2. Among them, $1^{\prime}-\mathrm{NH}$ derivatives 32, 34 and 35 exhibited improved antibacterial activities against $S$. pneumoniae with erm gene compared with the corresponding $1^{\prime}$ - $N$-methyl analogs (1,3 and 37), respectively. Because 34 and 35 especially showed enhanced antibacterial activities against the target pathogens, we found that double modifications at the C-6 and C-7 positions were important to improve antibacterial activities against S. pneumoniae with erm gene.
SAR analysis of antibacterial activities (MIC, $\mu \mathrm{g} \mathrm{ml}^{-1}$ ) of 7-Ssubstituted $1^{\prime}-N$-modified LCM derivatives $(38,40$ and $42-45)$

For the purpose of accumulating detail information of SAR at the 1 '-position, we synthesized novel LCM derivatives possessing various substituents at the $1^{\prime}$-position. At this point, the 5-(2-nitrophenyl)1,3,4-thiadiazol-2-yl group and the 4-(5-methylamino-thiazole-4-yl)1,3,4-thiadiazol-2-yl group were selected as a 7-substituent because of their SAR analysis (Tables 1 and 2). Consequently, compounds 40 and 42, possessing a 2-hydroxyethyl group at the 1'-position, showed antibacterial activities against target pathogens as shown in Table 3, but we concluded that it was difficult to enhance antibacterial activities against $S$. pneumoniae with erm gene by introducing an alternative 
<smiles>CC(C)CC(C)C</smiles>

70

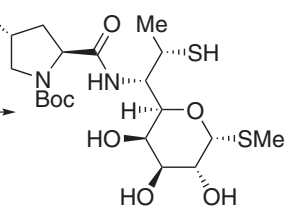

71

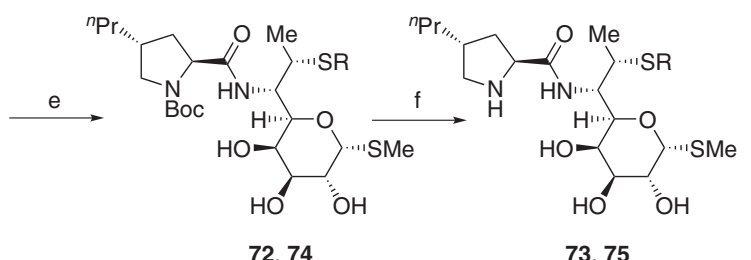

72,74

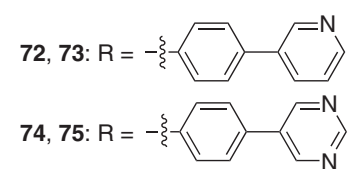

Scheme 7 Synthesis of 73 and 75. Conditions: (a) $\mathrm{MsCl}, \mathrm{NEt}_{3}, \mathrm{CH}_{2} \mathrm{Cl}_{2}, 0^{\circ}$ C, $1 \mathrm{~h}$; (b) AcSK, DMF, $60^{\circ} \mathrm{C}, 10 \mathrm{~h}$; (c) $1 \mathrm{~N} \mathrm{HCl}$, MeOH, r.t., $1 \mathrm{~h}$; (d) $\mathrm{NaOMe}, \mathrm{MeOH}$, r.t., $1.5 \mathrm{~h}$; (e) 3-(4-bromophenyl)pyridine or 5-(4bromophenyl)pyrimidine, $\mathrm{Pd}_{2}(\mathrm{DBA})_{3}$, Xantphos, $i-\mathrm{Pr}_{2} \mathrm{NEt}$, dioxane, reflux, 5-6 h; (f) TFA, $\mathrm{CH}_{2} \mathrm{Cl}_{2},-20^{\circ} \mathrm{C}$ to r.t., 3-5.5 h. DMF, dimethylformamide; r.t., room temperature; TFA, trifluoroacetic acid; Xantphos, 4,5-bis(diphenylphosphino)-9,9-dimethylxanthene, $\mathrm{Pd}_{2}(\mathrm{DBA})_{3}$, tris(dibenzylideneacetone)dipalladium(0).

substituent at the $1^{\prime}$-position, except a hydrogen atom or a methyl group. These results were closely related to SAR of $1^{\prime}-N$-alkyl-1'demethyllincomycin. ${ }^{17,21,24}$ Then, we selected a hydrogen atom and a methyl group at the $1^{\prime}$-position for modification of the proline moiety.

\section{SAR analysis of 7-S-substituted $1^{\prime}-N$ - and $4^{\prime}$-modified LCM derivatives (48 and 53-59) and TEL}

To confirm whether 7-S-substituted LCM analogs show similar antibacterial spectra to those reported previously, we synthesized $1^{\prime}$-demethyllincomycin derivatives possessing various substituents at the $4^{\prime}$-position. Structures of $7-S$-substituents followed those in Table 3. As shown in Table 4, compounds 56 and 57, possessing an $n$-pentyl group instead of an $n$-propyl group at the $4^{\prime}$-position, exhibited strong antibacterial activities against resistant bacteria with erm gene. Although antibacterial activities of TEL against S. pneumoniae with erm gene were stronger than those of 56, antibacterial activities of $\mathbf{5 6}$ against $S$. pyogenes with erm gene and resistant bacteria with mef gene were remarkably stronger than those of TEL.

\section{SAR analysis of 7-S-substituted $1^{\prime}-N$ - and $4^{\prime}$-modified LCM} derivatives with an alternative $7-S$-substituent $(67-69,73$ and 75$)$ We have reported significant potent antibacterial activities of LCM derivatives possessing a substituted phenyl group at the C-7 position so far. ${ }^{40}$ This time, we transformed the proline moiety (the $1^{\prime}$-and $4^{\prime}$ position) of 7-S-substituted phenyl derivatives as shown in Table 5, such as 7(S)-7-\{4-(morphorinocarbonyl)phenylthio\}lincomycin (76), $7(S)-7-\{4$-(pyridin-3-yl)phenylthio\}lincomycin (77) and 7(S)-7-\{4(pyrimidin-5-yl)phenylthio\}lincomycin (78). Their antibacterial activities are shown in Table 5. The $n$-pentyl analogs 68 and 69 could not exhibit improved antibacterial activities against $S$. pneumoniae and $S$. pyogenes with erm gene compared with 56 and $\mathbf{5 7}$. On the other hand, $1^{\prime}-\mathrm{NH}$ LCM derivatives $\mathbf{7 3}$ and $\mathbf{7 5}$ with a 4-(pyridin-3-yl)phenyl group and a 4-(pyrimidin-5-yl)phenyl group, respectively, exhibited markedly potent antibacterial activities against $S$. pneumoniae with erm gene. We confirmed that combination modification at the C-6 position (the proline moiety) and the C-7 position was important to enhance antibacterial activities against $S$. pneumoniae and $S$. pyogenes with erm gene.

\section{CONCLUSION}

To modify both the C-6 position (the proline moiety) and the C-7 position of LCM, we firstly prepared various substituted proline intermediates. The intermediates were coupled with MTL to give a wide variety of $1^{\prime}-N$-Boc-1'-demethyllincomycin derivatives. The 7-Ssubstituents were introduced as follows. Key intermediates 23-26, 36, 47 and $\mathbf{5 0}$ were synthesized by the Mitsunobu reaction ${ }^{37,38}$ with the corresponding thiol. Other key intermediates 23 and 27 were transformed to 7-S-benzoate by an $S_{N} 2$ reaction ${ }^{37,38}$ via Ms derivatives, and the benzoate moiety was finally converted to a 4-(morpholinocarbonyl)phenyl moiety (Scheme 6). 7(S)-7-Deoxy-7thiolincomycin (71) was coupled with biaryl bromide under the Pd-catalyzed cross-coupling reaction (Scheme 7). ${ }^{40,50}$ Compounds $\mathbf{3 8}$, 40 and $\mathbf{4 2 - 4 5}$ were also prepared from 34 or 35 . Those methodologies were found to be very practical to synthesize various LCM analogs modified at the C-6 and -7 positions.

By SAR analysis of combination modification at the $1^{\prime}$ - and 7-position, we concluded that it was difficult to enhance antibacterial activities against $S$. pneumoniae with erm gene by introducing an alternative substituent at the $1^{\prime}$-position, except a hydrogen atom or a methyl group. Then, we selected a hydrogen atom and a methyl group at the 1'-position for further modification of the proline moiety. We next modified the $4^{\prime}$-position (in the proline moiety) of LCM derivatives possessing a 5-(2-nitrophenyl)-1,3,4-thiadiazol-2-yl group or a 4-(5-methylamino-thiazol-4-yl)-1,3,4-thiadiazol-2-yl group at the C-7 position (Table 4). Compounds 56 and 57, possessing an $n$-pentyl group instead of an $n$-propyl group at the 4 -position, exhibited strong antibacterial activities against resistant bacteria with erm gene. Although antibacterial activities of TEL against $S$. pneumoniae with erm gene were stronger than those of 56, antibacterial activities of 56 against $S$. pyogenes with erm gene and resistant bacteria with mef gene were remarkably stronger than those of TEL. We found that combination modification at the C-6 position (the proline moiety) and the C-7 position was quite important to enhance antibacterial activities against $S$. pneumoniae and $S$. pyogenes with erm gene. The above SAR might be partially related to the polarity or water solubility of a molecule, but we only have limited SAR information. Further combination modification at the C-6 and C-7 positions of LCM analogs is in progress.

\section{EXPERIMENTAL PROCEDURES}

\section{General methods}

${ }^{1} \mathrm{H}$ NMR spectra were measured with a BRUKER Ascend 400 NMR spectrometer (Bruker Corporation, Coventry, UK) for $400 \mathrm{MHz}$, JEOL JNM-GSX 400 NMR spectrometer (JEOL,Tokyo, Japan) for $400 \mathrm{MHz}$ or a Varian Gemini 300 NMR spectrometer (Varian, Palo Alto, CA, USA) for $300 \mathrm{MHz}$ in $\mathrm{CDCl}_{3}$ or $\mathrm{CD}_{3} \mathrm{OD}$. TMS (0 p.p.m.) in $\mathrm{CDCl}_{3}$ or $\mathrm{CD}_{3} \mathrm{OD}$ was used as an internal reference standard. Mass spectra (MS) were obtained on a JEOL JMS-700 mass spectrometer (JEOL) or Agilent Technologies 6530-Q-TOF LC/MS mass spectrometer (Agilent Technologies, Santa Clara, CA, USA). The optical rotations were recorded with Jasco P-2300 digital polarimeter (Jasco Corporation, Tokyo, Japan). Column chromatography was performed with silica gel (Wakogel C200; Wako Pure Chemical Industries, Osaka, Japan). Preparative thin layer chromatography was performed with silica gel (Merck, Darmstadt, Germany; TLC plates Silica gel 60 F254). All organic extracts were dried over 
Table 2 Anti-bacterial activities (MIC, $\mu \mathrm{g} \mathrm{ml}{ }^{-1}$ ) of 7-S-substituted $1^{\prime}-\mathrm{NH}$ LCM derivatives (32-35) and $\mathbf{1}^{\prime}-\mathrm{N}$-Me analog 37

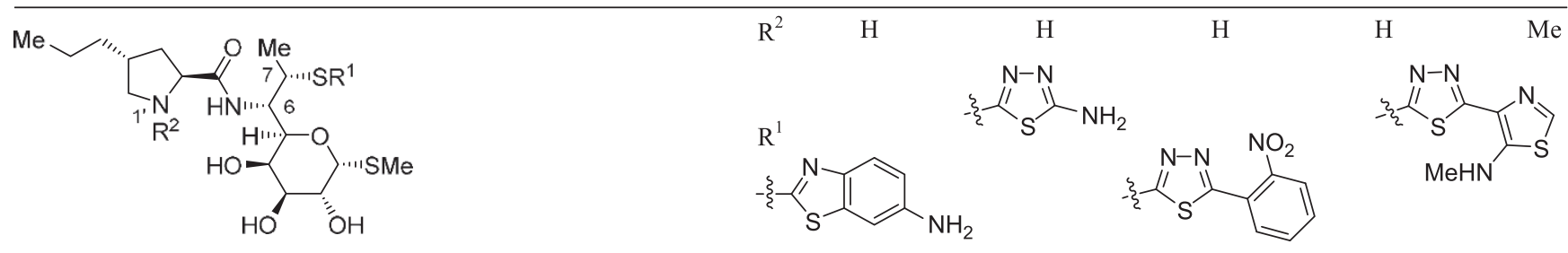

\begin{tabular}{|c|c|c|c|c|c|c|}
\hline Test organism ${ }^{\mathrm{a}}$ & Characteristics $^{b}$ & 32 & 33 & 34 & 35 & 37 \\
\hline Streptococcus pneumoniae DP1 type I & Susceptible & 0.06 & 0.5 & 0.03 & 0.12 & 0.12 \\
\hline S. pneumoniae-2 & Susceptible & 0.12 & 0.5 & 0.03 & 0.12 & 0.12 \\
\hline S. pneumoniae-3 & Susceptible & 0.03 & 0.5 & 0.03 & 0.12 & 0.12 \\
\hline S. pneumoniae-4 & ermAM methylase (c) & 8 & 128 & 1 & 1 & 2 \\
\hline S. pneumoniae-5 & ermAM methylase (c) & 64 & $>128$ & 2 & 2 & 4 \\
\hline S. pneumoniae- 6 & ermAM methylase $(c)+m e f E$ & 64 & $>128$ & 8 & 8 & 16 \\
\hline S. pneumoniae-7 & ermAM methylase (i) & 8 & 128 & 1 & 2 & 4 \\
\hline S. pneumoniae-8 & ermAM methylase (i) & 8 & 128 & 1 & 2 & 2 \\
\hline S. pneumoniae-9 & mefE efflux & 0.06 & 0.5 & 0.015 & 0.12 & 0.06 \\
\hline Streptococcus pyogenes Cook & Susceptible & 0.03 & 0.5 & 0.03 & 0.12 & 0.12 \\
\hline S. pyogenes-2 & ermAM methylase (c) & 8 & 32 & 1 & 1 & 2 \\
\hline S. pyogenes-3 & mefE efflux & 0.12 & 0.5 & 0.06 & 0.12 & 0.12 \\
\hline Haemophilus influenzae & Susceptible & 32 & 64 & 16 & 8 & 4 \\
\hline H. influenzae-2 & Susceptible & 32 & $>128$ & 8 & 8 & 4 \\
\hline H. influenzae-3 & Susceptible & 32 & 128 & 16 & 16 & 16 \\
\hline H. influenzae-4 & $\Delta \mathrm{acr}$ & 0.5 & 8 & 0.25 & 0.25 & 0.25 \\
\hline
\end{tabular}

Abbreviation: LCM, lincomycin.

aAll strains except standard organisms were clinically isolated.

$\mathrm{b}(\mathrm{c})$, constitutive; (i), inducible.

anhydrous $\mathrm{MgSO}_{4}$, and the solvent was removed with a rotary evaporator under reduced pressure.

$(2 S, 4 R)-1-N$-(tert-butoxycarbonyl)-4-n-propylpyrrolidine-2carboxylic acid (7)

To a solution of compound $4(1.0 \mathrm{~g}, 2.89 \mathrm{mmol})$ in $\mathrm{MeOH}(10 \mathrm{ml})$ was added $\mathrm{Pd} / \mathrm{C}(100 \mathrm{mg})$, and then vigorously stirred in hydrogen atmosphere at room temperature for $2 \mathrm{~h}$. The mixture was filtrated with celite, and then the solution was concentrated under reduced pressure. The resulting residue was filtrated with Chromatodisc $(0.45 \mu \mathrm{m})$ (Kurabo Industries, Osaka, Japan). The filtrated solution was concentrated under reduced pressure to obtain the title compound (745 mg, quant) as a colorless solid. FAB-MS $m / z 258(\mathrm{M}+\mathrm{H})^{+}$as $\mathrm{C}_{13} \mathrm{H}_{23} \mathrm{NO}_{4}$; ${ }^{1} \mathrm{H}$ NMR $\left.\left(400 \mathrm{MHz}, \mathrm{CD}_{3} \mathrm{OD}\right)\right)$ ) $0.70-0.93(\mathrm{~m}, 3 \mathrm{H}), 1.10-1.41(\mathrm{~m}, 13 \mathrm{H})$, $1.65-1.87(\mathrm{~m}, 1 \mathrm{H}), 1.93-2.06(\mathrm{~m}, 1 \mathrm{H}), 2.10-2.27(\mathrm{~m}, 1 \mathrm{H}), 2.73-2.89(\mathrm{~m}, 1 \mathrm{H})$, $3.43-3.63(\mathrm{~m}, 1 \mathrm{H})$ and $4.00-4.25(\mathrm{~m}, 1 \mathrm{H})$.

(2S,4R)-2-benzyl 1-tert-butyl 4-(3-hydroxypropyl)pyrrolidine-1,2dicarboxylate $(8)$

To a solution of compound $4(1.03 \mathrm{~g}, 2.98 \mathrm{mmol})$ in tetrahydrofuran (THF) $(3 \mathrm{ml})$ was added $0.5 \mathrm{M} 9$-BBN in THF solution $(8.95 \mathrm{ml}, 4.47 \mathrm{mmol})$ and stirred at $50{ }^{\circ} \mathrm{C}$ for $1 \mathrm{~h}$. Then, $1 \mathrm{~N} \mathrm{NaOH}(4 \mathrm{ml})$ and $35 \% \mathrm{H}_{2} \mathrm{O}_{2}(4 \mathrm{ml})$ were added to the mixture and stirred at $0{ }^{\circ} \mathrm{C}$ for $2 \mathrm{~h}$. The solution was added to the saturated aqueous $\mathrm{NaCl}$. The desired compound was extracted with ethyl acetate, and then the organic phase was dried over $\mathrm{Na}_{2} \mathrm{SO}_{4}$, filtrated and concentrated under reduced pressure. The resulting residue was purified by silica gel column chromatography (hexane/ethyl acetate $=3 / 1$ to $1 / 2$ ) to obtain the title compound ( $937 \mathrm{mg}, 87 \%$ ) as a colorless solid. EI-MS $m / z 363(\mathrm{M})^{+}$as $\mathrm{C}_{20} \mathrm{H}_{29} \mathrm{NO}_{5} ;{ }^{1} \mathrm{H}$ NMR $\left(400 \mathrm{MHz}, \mathrm{CDCl}_{3}\right) \delta 1.31-1.48(\mathrm{~m}, 11 \mathrm{H}), 1.49-1.60$ $(\mathrm{m}, 2 \mathrm{H}), 1.75-1.92(\mathrm{~m}, 1 \mathrm{H}), 2.05-2.14(\mathrm{~m}, 1 \mathrm{H}), 2.19-2.39(\mathrm{~m}, 1 \mathrm{H}), 2.88-3.05$ $(\mathrm{m}, 1 \mathrm{H}), 3.57-3.83(\mathrm{~m}, 3 \mathrm{H}), 4.25-4.50(\mathrm{~m}, 1 \mathrm{H}), 4.96-5.32(\mathrm{~m}, 2 \mathrm{H})$ and $7.27-7.47(\mathrm{~m}, 5 \mathrm{H})$.

(2S,4R)-2-Benzyl 1-tert-butyl 4-(3-(tert-butyldimethylsilyloxy) propyl)pyrrolidine-1,2-dicarboxylate (9)

To a solution of compound $8(2.80 \mathrm{~g}, 7.70 \mathrm{mmol})$ in dimethylformamide (DMF) $(15 \mathrm{ml})$ were added imidazole $(1.05 \mathrm{~g}, 15.4 \mathrm{mmol})$ and TBSCl $(1.74 \mathrm{~g}$, $11.56 \mathrm{mmol}$ ), and then stirred at room temperature for $30 \mathrm{~min}$. The mixture was extracted with ethyl acetate and then the organic phase was dried over $\mathrm{Na}_{2} \mathrm{SO}_{4}$, filtrated and concentrated under reduced pressure. The resulting residue was pumped up to obtain the title compound ( $3.50 \mathrm{~g}$, crude). The total amount of this compound was used without purification to synthesize 11.

(2S,4R)-2-benzyl 1-tert-butyl 4-(3-methoxypropyl)pyrrolidine-1,2dicarboxylate (10)

To a solution of compound 8 (900 mg, $2.48 \mathrm{mmol})$ in DMF (9 ml) was added $55 \% \mathrm{NaH}$ in oil $(99.2 \mathrm{mg}, 3.72 \mathrm{mmol})$ and stirred at room temperature for $30 \mathrm{~min}$. To the mixture was added methyl iodide $(924 \mu \mathrm{l}, 14.86 \mathrm{mmol})$ and then stirred at room temperature for $1 \mathrm{~h}$. The solution was added to the saturated aqueous $\mathrm{NaCl}$. The desired compound was extracted with ethyl acetate and then the organic phase was dried over $\mathrm{Na}_{2} \mathrm{SO}_{4}$, filtrated and concentrated under reduced pressure. The resulting residue was purified by silica gel column chromatography (hexane/ethyl acetate $=20 / 1$ to $4 / 1$ ) to obtain the title compound $(240 \mathrm{mg}, 26 \%)$ as a colorless oil. ESI-MS $\mathrm{m} / \mathrm{z} 378(\mathrm{M}+\mathrm{H})^{+}$ as $\mathrm{C}_{21} \mathrm{H}_{31} \mathrm{NO}_{5} ;{ }^{1} \mathrm{H}$ NMR $\left(400 \mathrm{MHz}, \mathrm{CDCl}_{3}\right) \delta 1.33,1.45$ (s x 2, 9H), 1.35-1.50 (m, 2H), 1.50-1.57 (m, 2H), 1.75-1.91 (m, 1H), 2.03-2.13 (m, 1H), 2.18-2.33 (m, 1H), 2.90-3.04 (m, 1H), $3.31(\mathrm{~s}, 3 \mathrm{H}), 3.25-3.40(\mathrm{~m}, 2 \mathrm{H}), 3.59-3.80$ $(\mathrm{m}, 1 \mathrm{H}), 4.25-4.47(\mathrm{~m}, 1 \mathrm{H}), 5.03-5.29(\mathrm{~m}, 2 \mathrm{H})$ and $7.29-7.42(\mathrm{~m}, 5 \mathrm{H})$. 


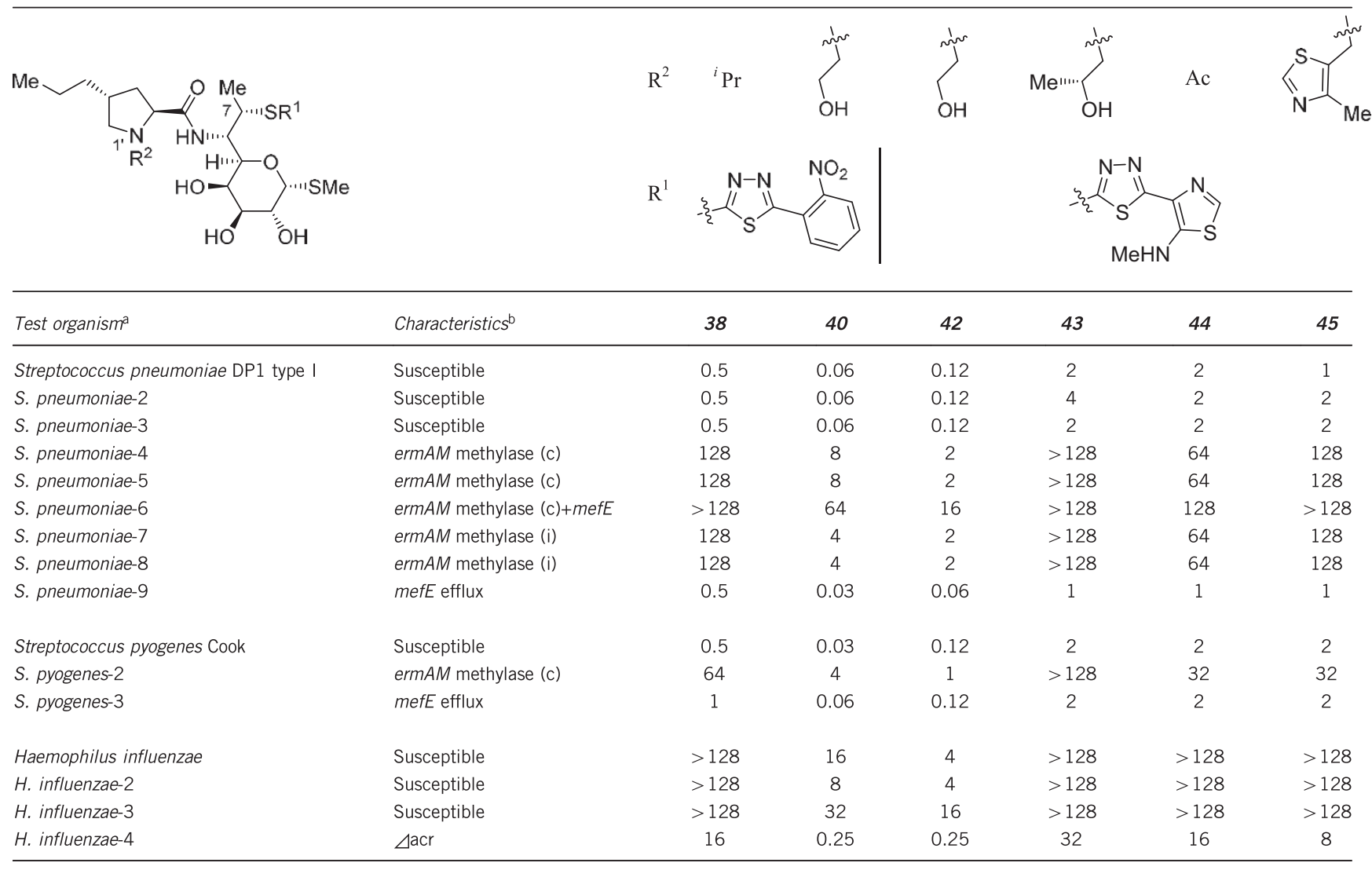

Abbreviation: LCM, lincomycin.

aAll strains except standard organisms were clinically isolated.

$\mathrm{b}(\mathrm{c})$, constitutive; (i), inducible.

(2S,4R)-1-N-(tert-butoxycarbonyl)-4-(3-(tert-butyldimethylsilyloxy) propyl)pyrrolidine-2-carboxylic acid (11)

Compound 9 ( $3.50 \mathrm{~g}$, crude) in $\mathrm{MeOH}(50 \mathrm{ml})$ were treated for $30 \mathrm{~min}$ according to the similar procedure as described for the preparation of 7 to afford 11 ( $3.02 \mathrm{~g}$, crude). The total amount of this compound was used to synthesize 49.

(2S,4R)-1-N-(tert-butoxycarbonyl)-4-(3-methoxypropyl) pyrrolidine-2-carboxylic acid (12)

Compound 10 ( $200 \mathrm{mg}, 0.530 \mathrm{mmol})$ in $\mathrm{MeOH}(2 \mathrm{ml})$ were treated for $1 \mathrm{~h}$ according to the similar procedure as described for the preparation of 7 to afford 12 (152 mg, crude). The total amount of this compound was used without purification to synthesize 19.

(2S,4R)-1- $N$-(tert-butoxycarbonyl)-4-i-butylpyrrolidine-2-carboxylic acid (13)

Compound 5 (195.3 mg, $0.54 \mathrm{mmol})$ in $\mathrm{MeOH}(2 \mathrm{ml})$ were treated for $30 \mathrm{~min}$ according to the similar procedure as described for the preparation of 7 to afford $13(141.1 \mathrm{mg}, 96 \%)$ as an off-white solid. ESI-MS $m / z 272(\mathrm{M}+\mathrm{H})^{+}$as $\mathrm{C}_{14} \mathrm{H}_{25} \mathrm{NO}_{4} ;{ }^{1} \mathrm{H}$ NMR $\left(400 \mathrm{MHz}, \mathrm{CD}_{3} \mathrm{OD}\right) \delta$ $0.90(\mathrm{~d}, J=6.7 \mathrm{~Hz}, 3 \mathrm{H}), 0.92(\mathrm{~d}, J=6.7 \mathrm{~Hz}, 3 \mathrm{H}), 1.23-1.35(\mathrm{~m}, 2 \mathrm{H}), 1.41$, $1.45(\mathrm{~s} \times 2,9 \mathrm{H}), 1.51-1.64(\mathrm{~m}, 1 \mathrm{H}), 1.76-1.95(\mathrm{~m}, 1 \mathrm{H}), \quad 2.07-2.15$ $(\mathrm{m}, 1 \mathrm{H}), 2.28-2.44(\mathrm{~m}, 1 \mathrm{H}), 2.85-3.00(\mathrm{~m}, 1 \mathrm{H}), 3.57-3.74(\mathrm{~m}, 1 \mathrm{H})$ and $4.18-4.32(\mathrm{~m}, 1 \mathrm{H})$.
(2S,4R)-1- $N$-(tert-butoxycarbonyl)-4-n-pentylpyrrolidine-2carboxylic acid (14)

Compound $6(1.69 \mathrm{~g}, 4.53 \mathrm{mmol})$ in $\mathrm{MeOH}(20 \mathrm{ml})$ were treated for $2 \mathrm{~h}$ according to the similar procedure as described for the preparation of 7 to afford $14(1.16 \mathrm{~g}, 89.5 \%)$ as a colorless solid. FAB-MS $\mathrm{m} / \mathrm{z} 286(\mathrm{M}+\mathrm{H})^{+}$as $\mathrm{C}_{15} \mathrm{H}_{27} \mathrm{NO}_{4} ;{ }^{1} \mathrm{H}$ NMR $\left(400 \mathrm{MHz}, \mathrm{CD}_{3} \mathrm{OD}\right) \delta 0.80-0.98$ (m, 3H), $1.20-1.51$ $(\mathrm{m}, 8 \mathrm{H}), 1.42,1.46(\mathrm{~s} \times 2,9 \mathrm{H}), 1.78-1.98(\mathrm{~m}, 1 \mathrm{H}), 2.10$ (ddd, $J=12.7,6.2$, $2.1 \mathrm{~Hz}, 1 \mathrm{H}), 2.19-2.36(\mathrm{~m}, 1 \mathrm{H}), 2.85-3.01(\mathrm{~m}, 1 \mathrm{H}), 3.57-3.75(\mathrm{~m}, 1 \mathrm{H})$, $4.17-4.33(\mathrm{~m}, 1 \mathrm{H})$.

(2S,4R)-1-N-(tert-butoxycarbonyl)-4-((E)-pent-2-enyl)pyrrolidine-2carboxylic acid (15)

To a solution of compound $6(2.0 \mathrm{~g}, 5.79 \mathrm{mmol})$ in $\mathrm{MeOH}(20 \mathrm{ml})$ was added $1 \mathrm{M}$ aqueous $\mathrm{NaOH}(20 \mathrm{ml})$ and stirred at room temperature for $22 \mathrm{~h}$. The mixture was diluted with $\mathrm{H}_{2} \mathrm{O}$ and $\mathrm{Et}_{2} \mathrm{O}$ and washed by $\mathrm{Et}_{2} \mathrm{O}$. Aqueous layer was added to the saturated aqueous citric acid, extracted with ethyl acetate and then the organic phase was washed with $\mathrm{H}_{2} \mathrm{O}$, dried over $\mathrm{Na}_{2} \mathrm{SO}_{4}$, filtrated and concentrated under reduced pressure to obtain the title compound $(1.55 \mathrm{~g}$, $94.5 \%)$ as a colorless solid. FAB-MS $m / z 284(\mathrm{M}+\mathrm{H})^{+}$as $\mathrm{C}_{15} \mathrm{H}_{25} \mathrm{NO}_{4} ;{ }^{1} \mathrm{H}$ NMR $\left(400 \mathrm{MHz}, \mathrm{CD}_{3} \mathrm{OD}\right) \delta$ 0.89-1.05 (m, 3H), $1.41(\mathrm{~s}, 6 \mathrm{H}), 1.45(\mathrm{~s}, 3 \mathrm{H}), 1.85-2.15$ $(\mathrm{m}, 6 \mathrm{H}), 2.23-2.39(\mathrm{~m}, 1 \mathrm{H}), 2.92-3.09(\mathrm{~m}, 1 \mathrm{H}), 3.52-3.68(\mathrm{~m}, 1 \mathrm{H}), 4.18-4.31$ $(\mathrm{m}, 1 \mathrm{H}), 5.30-5.44(\mathrm{~m}, 1 \mathrm{H})$ and $5.46-5.60(\mathrm{~m}, 1 \mathrm{H})$.

2(S)-1-N-(tert-butoxycarbonyl)-4,4-di- $n$-propylpyrrolidine-2carboxylic acid (17)

Compound $16(2.0 \mathrm{~g}, 5.19 \mathrm{mmol})$ in $\mathrm{MeOH}(23 \mathrm{ml})$ were treated for $4.5 \mathrm{~h}$ according to the similar procedure as described for the preparation of 7 to 
Table 4 Anti-bacterial activities (MIC, $\mu \mathrm{g} \mathrm{ml}^{-1}$ ) of 7-S-substituted $1^{\prime}-N$ - and $4^{\prime}$-modified LCM derivatives (48 and 53-59) and TEL

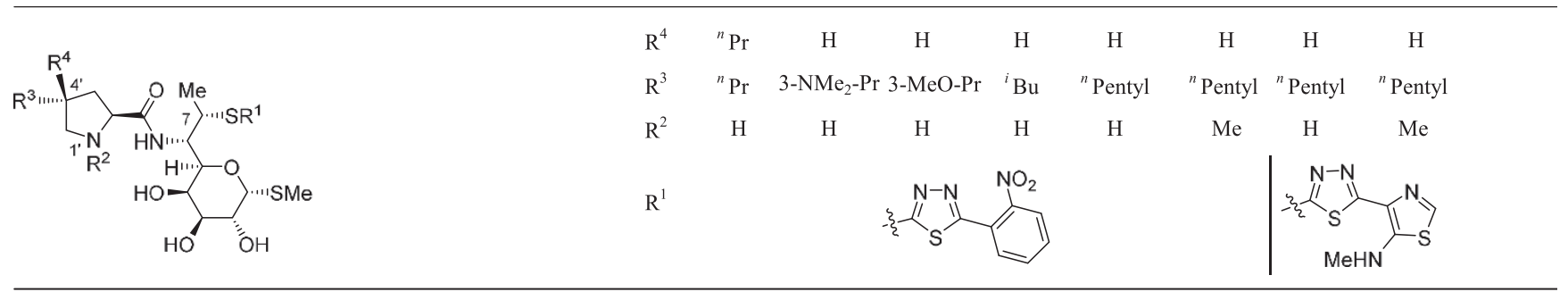

\begin{tabular}{|c|c|c|c|c|c|c|c|c|c|c|}
\hline Test organism ${ }^{a}$ & Characteristics $^{b}$ & 48 & 53 & 54 & 55 & 56 & 57 & 58 & 59 & TEL \\
\hline Streptococcus pneumoniae DP1 type I & Susceptible & 16 & 128 & 0.06 & 0.06 & $\leqq 0.008$ & $\leqq 0.008$ & 0.03 & 0.03 & $\leqq 0.008$ \\
\hline S. pneumoniae-2 & Susceptible & 16 & 128 & 0.06 & 0.06 & $\leqq 0.008$ & 0.015 & 0.06 & 0.06 & $\leqq 0.008$ \\
\hline S. pneumoniae-3 & Susceptible & 16 & 32 & 0.06 & 0.03 & $\leqq 0.008$ & $\leqq 0.008$ & NT & 0.015 & $\leqq 0.008$ \\
\hline S. pneumoniae-4 & ermAM methylase (c) & 64 & $>128$ & 32 & 32 & 0.5 & 1 & 1 & 2 & 0.5 \\
\hline S. pneumoniae-5 & ermAM methylase (c) & 64 & $>128$ & 16 & 32 & 0.5 & 1 & 2 & 4 & 2 \\
\hline S. pneumoniae- 6 & ermAM methylase $(c)+$ mefE & 64 & $>128$ & 64 & 128 & 2 & 4 & 4 & 8 & 1 \\
\hline S. pneumoniae-7 & ermAM methylase (i) & 64 & $>128$ & 8 & 8 & NT & NT & NT & NT & 0.03 \\
\hline S. pneumoniae-8 & ermAM methylase (i) & 64 & $>128$ & 8 & 8 & 0.5 & 0.5 & 1 & 2 & 0.03 \\
\hline S. pneumoniae-9 & mefE efflux & 16 & 64 & 0.06 & 0.03 & $\leqq 0.008$ & $\leqq 0.008$ & 0.06 & 0.015 & 0.06 \\
\hline Streptococcus pyogenes Cook & Susceptible & 2 & 8 & 0.06 & 0.03 & 0.015 & 0.015 & 0.12 & 0.06 & $\leqq 0.008$ \\
\hline S. pyogenes-2 & ermAM methylase (c) & 64 & $>128$ & 8 & 8 & 0.5 & 0.5 & 0.5 & 0.5 & 16 \\
\hline S. pyogenes-3 & mefE efflux & 8 & 8 & 0.12 & 0.06 & 0.015 & 0.03 & 0.06 & 0.06 & 0.25 \\
\hline Haemophilus influenzae & Susceptible & $>128$ & $>128$ & 128 & 64 & 8 & 8 & 16 & 8 & 0.5 \\
\hline H. influenzae-2 & Susceptible & 128 & $>128$ & 128 & 32 & 8 & 4 & 8 & 4 & 2 \\
\hline H. influenzae-3 & Susceptible & $>128$ & $>128$ & $>128$ & 128 & 16 & 8 & 16 & 8 & 1 \\
\hline H. influenzae-4 & $\Delta \mathrm{acr}$ & 32 & $>128$ & 2 & 2 & 0.25 & 0.06 & 0.25 & 0.12 & 0.25 \\
\hline
\end{tabular}

Abbreviations: LCM, lincomycin; NT, not tested; TEL, telithromycin.

aAll strains except standard organisms were clinically isolated.

b(c): constitutive; (i): inducible.

afford 17 (1.55 g, quant) as an off-white oil. ESI-MS $m / z 300(\mathrm{M}+\mathrm{H})^{+}$as $\mathrm{C}_{16} \mathrm{H}_{29} \mathrm{NO}_{4} ;{ }^{1} \mathrm{H}$ NMR $\left(400 \mathrm{MHz}, \mathrm{CD}_{3} \mathrm{OD}\right) \delta 0.91(\mathrm{t}, J=6.9 \mathrm{~Hz}, 3 \mathrm{H}), 0.93$

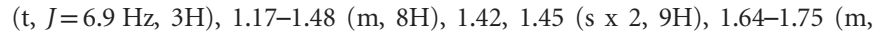
$1 \mathrm{H}), 2.11-2.24(\mathrm{~m}, 1 \mathrm{H}), 3.10(\mathrm{~d}, J=10.6 \mathrm{~Hz}, 1 \mathrm{H}), 3.35-3.42(\mathrm{~m}, 1 \mathrm{H})$, $4.01-4.25(\mathrm{~m}, 1 \mathrm{H})$.

\section{$1^{\prime}$-N-(tert-butoxycarbonyl)-1'-demethyllincomycin (18)}

To a solution of compound $7(745.0 \mathrm{mg}, 2.90 \mathrm{mmol})$ in DMF $(27 \mathrm{ml})$ were added 1-hydroxybenzotriazole (469.4 mg, $3.47 \mathrm{mmol}$ ), $N, N^{\prime}$-dicyclohexylcarbodiimide $(716.8 \mathrm{mg}, 3.47 \mathrm{mmol})$ and MTL $(1.10 \mathrm{~g}, 4.34 \mathrm{mmol})$ and stirred at room temperature for $15 \mathrm{~h}$. The mixture was added to $\mathrm{H}_{2} \mathrm{O}$ and ethyl acetate. The desired compound was extracted with ethyl acetate, and then the organic phase was washed with $\mathrm{H}_{2} \mathrm{O}$, dried over $\mathrm{Na}_{2} \mathrm{SO}_{4}$, filtrated and concentrated under reduced pressure to obtain the title compound. The total amount of this compound was used without purification to synthesize 23 . For the qualified analytical purpose, the above crude compound $\mathbf{1 8}$ was purified by column chromatography (ethyl acetate only) to obtain the title compound as a colorless solid. FAB-MS $\mathrm{m} / \mathrm{z} 493(\mathrm{M}+\mathrm{H})^{+}$as $\mathrm{C}_{22} \mathrm{H}_{40} \mathrm{~N}_{2} \mathrm{O}_{8} \mathrm{~S} ;{ }^{1} \mathrm{H}$ NMR $(400 \mathrm{MHz}$, $\left.\mathrm{CD}_{3} \mathrm{OD}\right) \delta 0.78-0.93(\mathrm{~m}, 3 \mathrm{H}), 1.05-1.45(\mathrm{~m}, 7 \mathrm{H}), 1.35,1.38(\mathrm{~s} \times 2,9 \mathrm{H})$, 1.59-1.81 (m, 1H), 1.88-2.09 (m, 1H), $1.97(\mathrm{~s}, 3 \mathrm{H}), 2.23-2.43(\mathrm{~m}, 1 \mathrm{H}), 2.83$ (br t, $J=10.1 \mathrm{~Hz}, 1 \mathrm{H}), 3.43-3.63(\mathrm{~m}, 2 \mathrm{H}), 3.64-3.82(\mathrm{~m}, 1 \mathrm{H}), 3.84-4.09$ (m, 3H), 4.10-4.19 (m, 1H), 4.21-4.39 (m, 1H) and $5.14($ br d, $J=5.4 \mathrm{~Hz}, 1 \mathrm{H})$.

\section{$1^{\prime}$-N-(tert-butoxycarbonyl)-1'-demethyl-4'-depropyl-4' -(3-} methoxypropyl)lincomycin (19)

To a solution of compound $12(152.3 \mathrm{mg}, 0.53 \mathrm{mmol})$ in DMF $(1.5 \mathrm{ml})$ were added 1-hydroxybenzotriazole $\quad(93.0 \mathrm{mg}, \quad 0.689 \mathrm{mmol}), \quad \mathrm{N}, \mathrm{N}$ '-dicyclohexylcarbodiimide (142.0 mg, $0.689 \mathrm{mmol}$ ) and MTL (174.0 mg, $0.689 \mathrm{mmol}$ ) and stirred at room temperature for $4.5 \mathrm{~h}$. The mixture was added to $\mathrm{H}_{2} \mathrm{O}$ and ethyl acetate. The desired compound was extracted with ethyl acetate, and then the organic phase was washed with $\mathrm{H}_{2} \mathrm{O}$, dried over $\mathrm{Na}_{2} \mathrm{SO}_{4}$, filtrated and concentrated under reduced pressure. The resulting residue was purified by silica gel column chromatography (hexane/ethyl acetate $=1 / 1$ to ethyl acetate only to ethyl acetate/ $\mathrm{MeOH}=24 / 1$ ) to obtain the title compound (245.0 mg, $88.5 \%$ in two steps from 10) as a colorless solid. ESI-MS $m / z 523(\mathrm{M}+\mathrm{H})^{+}$as $\mathrm{C}_{23} \mathrm{H}_{42} \mathrm{~N}_{2} \mathrm{O}_{9} \mathrm{~S} ;{ }^{1} \mathrm{H}$ NMR $\left(400 \mathrm{MHz}, \mathrm{CD}_{3} \mathrm{OD}\right)$

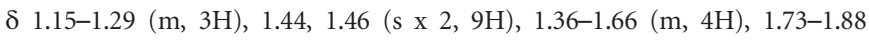
$(\mathrm{m}, 1 \mathrm{H}), 2.06,2.07(\mathrm{~s} \times 2,3 \mathrm{H}), 2.09-2.17(\mathrm{~m}, 1 \mathrm{H}), 2.33-2.50(\mathrm{~m}, 1 \mathrm{H}), 2.93$ (br t, $J=9.9 \mathrm{~Hz}, 1 \mathrm{H}), 3.32(\mathrm{~s}, 3 \mathrm{H}), 3.40(\mathrm{t}, J=6.3 \mathrm{~Hz}, 2 \mathrm{H}), 3.53-3.96(\mathrm{~m}, 3 \mathrm{H})$, 3.98-4.18 (m, 3H), 4.20-4.26 (m, $1 \mathrm{H}), 4.28-4.48(\mathrm{~m}, 1 \mathrm{H})$ and 5.24 $(\mathrm{d}, J=5.6 \mathrm{~Hz}, 1 \mathrm{H})$.

\section{$1^{\prime}$-N-(tert-butoxycarbonyl)-1'-demethyl-4'-depropyl-4'-i-} butyllincomycin (20)

To a solution of compound $13(57.9 \mathrm{mg}, 0.21 \mathrm{mmol})$ in DMF $(1.0 \mathrm{ml})$ were added 1-hydroxybenzotriazole $(43.2 \mathrm{mg}, 0.32 \mathrm{mmol}), N, N^{\prime}$-dicyclohexylcarbodiimide (61.4 g, $0.32 \mathrm{mmol}$ ) and MTL ( $81.1 \mathrm{mg}, 0.32 \mathrm{mmol})$ and stirred at room temperature for $1 \mathrm{~h}$. The mixture was added to the saturated aqueous $\mathrm{NaHCO}_{3}$ and ethyl acetate. The desired compound was extracted with ethyl acetate, and then the organic phase was dried over $\mathrm{Na}_{2} \mathrm{SO}_{4}$, filtrated and concentrated under reduced pressure. The resulting residue was purified by silica gel column chromatography (chloroform $/ \mathrm{MeOH}=10 / 1$ ) to obtain the title compound (100.2 mg, 92.7\%) as a colorless solid. FAB-MS $\mathrm{m} / \mathrm{z} 507$ $(\mathrm{M}+\mathrm{H})^{+}$as $\mathrm{C}_{23} \mathrm{H}_{42} \mathrm{~N}_{2} \mathrm{O}_{8} \mathrm{~S} ;{ }^{1} \mathrm{H}$ NMR $\left(400 \mathrm{MHz}, \mathrm{CD}_{3} \mathrm{OD}\right) \delta$ 0.87-0.98 (m, 6H), 
Table 5 Anti-bacterial activities (MIC, $\mu \mathrm{g} \mathrm{ml}^{-1}$ ) of 7-S-substituted $\mathbf{1}^{\prime}$ - $\mathrm{N}$ - and $4^{\prime}$-modified LCM derivatives with an alternative 7-S-substituent (67-69, 73 and 75)

\begin{tabular}{|c|c|c|c|c|c|c|c|c|c|}
\hline \multirow{3}{*}{ (n) } & \multirow{2}{*}{$\begin{array}{l}\mathrm{R}^{3} \\
\mathrm{R}^{2} \\
\mathrm{R}^{1}\end{array}$} & \multicolumn{2}{|c|}{${ }^{n} \operatorname{Pr}$} & \multicolumn{2}{|c|}{${ }^{n}$ Pentyl } & \multicolumn{2}{|c|}{${ }^{n} \operatorname{Pr}$} & \multicolumn{2}{|c|}{${ }^{n} \mathrm{Pr}$} \\
\hline & & $\mathrm{H}$ & $\mathrm{Me}$ & $\mathrm{H}$ & $\mathrm{Me}$ & $\mathrm{H}$ & $\mathrm{Me}$ & $\mathrm{H}$ & $\mathrm{Me}$ \\
\hline & Characteristics $^{b}$ & 67 & $76^{c}$ & 68 & 69 & 73 & $77^{c}$ & 75 & $78^{\mathrm{c}}$ \\
\hline Streptococcus pneumoniae DP1 type I & Susceptible & 0.25 & 0.06 & NT & NT & 0.015 & $\leqq 0.008$ & $\leqq 0.008$ & $\leqq 0.008$ \\
\hline S. pneumoniae-2 & Susceptible & 0.25 & 0.06 & 0.12 & 0.06 & 0.015 & $\leqq 0.008$ & $\leqq 0.008$ & $\leqq 0.008$ \\
\hline S. pneumoniae-3 & Susceptible & 0.25 & 0.06 & 0.25 & 0.12 & 0.03 & 0.015 & $\leqq 0.008$ & $\leqq 0.008$ \\
\hline S. pneumoniae-4 & ermAM methylase (c) & 16 & 8 & 4 & 4 & 1 & 0.5 & 0.5 & 0.5 \\
\hline S. pneumoniae- 5 & ermAM methylase (c) & 16 & 2 & 8 & 4 & 2 & 1 & NT & 1 \\
\hline S. pneumoniae-6 & ermAM methylase $(\mathrm{c})+m e f E$ & 64 & 8 & 16 & 16 & 4 & 2 & 2 & 2 \\
\hline S. pneumoniae-7 & ermAM methylase (i) & 4 & 2 & 2 & 1 & 0.5 & 0.25 & 0.25 & 0.25 \\
\hline S. pneumoniae-8 & ermAM methylase (i) & 8 & 1 & 0.5 & 0.5 & 0.5 & 0.25 & 0.25 & 0.25 \\
\hline S. pneumoniae-9 & mefE efflux & 0.25 & 0.03 & 0.06 & 0.015 & $\leqq 0.008$ & $\leqq 0.008$ & $\leqq 0.008$ & $\leqq 0.008$ \\
\hline Streptococcus pyogenes Cook & Susceptible & 0.12 & 0.06 & 0.12 & 0.12 & 0.03 & 0.015 & $\leqq 0.008$ & $\leqq 0.008$ \\
\hline S. pyogenes-2 & ermAM methylase (c) & 4 & 4 & 0.5 & 0.5 & 0.5 & 0.5 & 0.5 & 0.5 \\
\hline S. pyogenes-3 & mefE efflux & 0.25 & 0.06 & 0.12 & 0.06 & 0.03 & 0.015 & $\leqq 0.008$ & 0.015 \\
\hline Haemophilus influenzae & Susceptible & 32 & 4 & 32 & 8 & 8 & 4 & 8 & 4 \\
\hline H. influenzae-2 & Susceptible & 64 & 4 & 32 & 8 & 8 & 2 & 4 & 2 \\
\hline H. influenzae-3 & Susceptible & 128 & 16 & 32 & 16 & 16 & 16 & 8 & 8 \\
\hline H. influenzae-4 & $\Delta \mathrm{acr}$ & 2 & 0.25 & 1 & 0.12 & 0.25 & 0.06 & 0.12 & 0.06 \\
\hline
\end{tabular}

Abbreviations: LCM, lincomycin; NT, not tested.

aAll strains except standard organisms were clinically isolated.

b (c), constitutive; (i), inducible.

'Previously reported.
chis , constitutive (i),

$1.16-1.32(\mathrm{~m}, 6 \mathrm{H}), 1.44,1.47$ ( $\mathrm{x}$ x $2,9 \mathrm{H}), 1.51-1.66(\mathrm{~m}, 1 \mathrm{H}), 1.70-1.86$ $(\mathrm{m}, 1 \mathrm{H}), 2.05,2.06(\mathrm{~s} \times 2,3 \mathrm{H}), 2.07-2.15(\mathrm{~m}, 1 \mathrm{H}), 2.39-2.57(\mathrm{~m}, 1 \mathrm{H}), 2.90$ $(\mathrm{t}, J=10.1 \mathrm{~Hz}, 1 \mathrm{H}), 3.55-3.71(\mathrm{~m}, 1 \mathrm{H}), 3.72-3.88(\mathrm{~m}, 1 \mathrm{H}), 3.98-4.19(\mathrm{~m}, 2 \mathrm{H})$, $4.23(\mathrm{~d}, J=8.8 \mathrm{~Hz}, 1 \mathrm{H}), 4.32-4.51(\mathrm{~m}, 1 \mathrm{H})$ and $5.23(\mathrm{~d}, J=5.4 \mathrm{~Hz}, 1 \mathrm{H})$.

\section{$1^{\prime}$-N-(tert-butoxycarbonyl)-1'-demethyl-4'-depropyl-4'-n- pentyllincomycin (21)}

Compound 14 (1.16 g, $4.05 \mathrm{mmol}), \quad$ 1-hydroxybenzotriazole $(820 \mathrm{mg}$, $6.07 \mathrm{mmol}), N, N^{\prime}$-dicyclohexylcarbodiimide $(1.25 \mathrm{~g}, 6.07 \mathrm{mmol})$ and MTL $(1.54 \mathrm{~g}, 6.07 \mathrm{mmol})$ in DMF $(15.0 \mathrm{ml})$ were treated for $23 \mathrm{~h}$ according to the similar procedure as described for the preparation of $\mathbf{1 8}$ to afford $\mathbf{2 1}$. The total amount of this compound was used without purification to synthesize 26.

$1^{\prime}$-N-(tert-butoxycarbonyl)-1'-demethyl-4' -depropyl-4'-\{(E)-pent-2enyl\}lincomycin (22)

Compound $15 \quad(1.55 \mathrm{~g}, \quad 5.47 \mathrm{mmol}), \quad$ 1-hydroxybenzotriazole $(1.11 \mathrm{~g}$, $8.21 \mathrm{mmol}), N, N^{\prime}$-dicyclohexylcarbodiimide $(1.69 \mathrm{~g}, 8.21 \mathrm{mmol})$ and MTL $(2.08 \mathrm{mg}, 8.21 \mathrm{mmol})$ in DMF $(23 \mathrm{ml})$ were treated for $3 \mathrm{~h}$ according to the similar procedure as described for the preparation of $\mathbf{1 8}$ to afford $\mathbf{2 2}$. The total amount of this compound was used without purification to synthesize 27.

\section{$\mathbf{1}^{\prime}$ - $\mathrm{N}$-(tert-butoxycarbonyl)-1' -demethyl-2,3,4-tris-O-(trimethylsilyl)} lincomycin (23)

To a solution of compound $\mathbf{1 8}$ (crude) in pyridine $(15 \mathrm{ml}$ ) were added trimethylchlorosilane $(1.85 \mathrm{ml}, 14.5 \mathrm{mmol})$ and hexamethyldisilazane $(3.03 \mathrm{ml}$, $14.5 \mathrm{mmol}$ ) and stirred at room temperature for $30 \mathrm{~min}$, and then the solution was added to the saturated aqueous $\mathrm{NaHCO}_{3}$. The desired compound was extracted with ethyl acetate, washed with $\mathrm{H}_{2} \mathrm{O}$ and then the organic phase was dried over $\mathrm{Na}_{2} \mathrm{SO}_{4}$, filtrated and concentrated under reduced pressure. To the resulting residue were added methanol $(16.4 \mathrm{ml})$ and $6 \mathrm{~N}$ acetic acid $(0.87 \mathrm{ml})$, and stirred at room temperature for $11 \mathrm{~h}$. The mixture was added to the saturated aqueous $\mathrm{NaHCO}_{3}$ and concentrated under reduced pressure to remove $\mathrm{MeOH}$. The desired compound was extracted with ethyl acetate, and then the organic phase was dried over $\mathrm{Na}_{2} \mathrm{SO}_{4}$, filtrated and concentrated under reduced pressure. The resulting residue was purified by silica gel column chromatography (hexane/ethyl acetate $=2 / 1$ to $1 / 2$ ) to obtain the title compound $(1.45 \mathrm{~g}, 70.6 \%$ in three steps from 7 ) as a colorless solid. ESI-MS $\mathrm{m} / \mathrm{z}$ $709(\mathrm{M}+\mathrm{H})^{+}$as $\mathrm{C}_{31} \mathrm{H}_{64} \mathrm{~N}_{2} \mathrm{O}_{8} \mathrm{SSi}_{3} ;{ }^{1} \mathrm{H}$ NMR $\left(400 \mathrm{MHz}, \mathrm{CD}_{3} \mathrm{OD}\right) \delta 0.12-0.26$ (m, 27H), 0.85-1.00 (m, 3H), 1.07-1.25 (m, 3H), 1.26-1.41 (m, 4H), 1.44, 1.46 (s x 2, 9H), 1.66-1.93 (m, 1H), 2.03, $2.05(\mathrm{~s} \mathrm{x} \mathrm{2,} \mathrm{3H),} \mathrm{1.98-2.42} \mathrm{(m,} \mathrm{2H),}$ $2.88-3.02(\mathrm{~m}, 1 \mathrm{H}), 3.54-4.40(\mathrm{~m}, 8 \mathrm{H})$ and $5.18(\mathrm{~d}, J=5.4 \mathrm{~Hz}, 1 \mathrm{H})$.

\section{$1^{\prime}$-N-(tert-butoxycarbonyl)-1'-demethyl-4'-depropyl-4'-}

(3-methoxypropyl)-2,3,4-tris-O-(trimethylsilyl)lincomycin (24)

Compound $19(290 \mathrm{mg}, 0.56 \mathrm{mmol})$, trimethylchlorosilane $(355 \mu \mathrm{l}$, $2.77 \mathrm{mmol})$ and hexamethyldisilazane $(581 \mu \mathrm{l}, 2.77 \mathrm{mmol})$ in pyridine $(1.0 \mathrm{ml})$ were treated for $1.0 \mathrm{~h}$ according to the similar procedure as described for the preparation of $\mathbf{2 3}$, and then the crude compound and $6 \mathrm{~N}$ acetic acid $(167 \mu \mathrm{l})$ in methanol $(3.1 \mathrm{ml})$ were treated for $30 \mathrm{~min}$ according to the similar procedure as described for the preparation of 23 to afford $24(282 \mathrm{mg}, 68.7 \%$ in two steps from 19) as a colorless solid. ESI-MS $m / z 739(\mathrm{M}+\mathrm{H})^{+}$as $\mathrm{C}_{32} \mathrm{H}_{66} \mathrm{~N}_{2} \mathrm{O}_{9} \mathrm{SSi}_{3}$. 
$1^{\prime}$ - $N$-(tert-butoxycarbonyl)-1'-demethyl-4' -depropyl-4'-i-butyl2,3,4-tris-O-(trimethylsilyl)lincomycin (25)

Compound $20 \quad(652 \mathrm{mg}, 1.63 \mathrm{mmol})$, trimethylchlorosilane $(1.02 \mathrm{ml}$, $8.02 \mathrm{mmol})$ and hexamethyldisilazane $(1.68 \mathrm{ml}, 8.02 \mathrm{mmol})$ in pyridine $(3.5 \mathrm{ml})$ were treated for $1 \mathrm{~h}$ according to the similar procedure as described for the preparation of 23 , and then the crude compound and $6 \mathrm{~N}$ acetic acid $(480 \mu \mathrm{l})$ in methanol $(9 \mathrm{ml})$ were treated for $30 \mathrm{~min}$ according to the similar procedure as described for the preparation of $\mathbf{2 3}$ to afford $\mathbf{2 5}$ (946 $\mathrm{mg}$, 79.8\% in two steps from 20) as a colorless solid. FAB-MS $\mathrm{m} / \mathrm{z} 723(\mathrm{M}+\mathrm{H})^{+}$as $\mathrm{C}_{32} \mathrm{H}_{66} \mathrm{~N}_{2} \mathrm{O}_{8} \mathrm{SSi}_{3} ;{ }^{1} \mathrm{H}$ NMR $\left(400 \mathrm{MHz}, \mathrm{CDCl}_{3}\right) \delta 0.11-0.21(\mathrm{~m}, 27 \mathrm{H}), 0.90$ $(\mathrm{d}, J=6.6 \mathrm{~Hz}, 6 \mathrm{H}), 1.05-1.33(\mathrm{~m}, 5 \mathrm{H}), 1.49(\mathrm{~s}, 9 \mathrm{H}), 1.50-1.61(\mathrm{~m}, 2 \mathrm{H}), 2.07$ (s, 3H), 2.13-2.57 (m, 1H), 2.72-3.13 (m, 1H), 3.40-3.82 (m, 3H), 3.94-4.19 $(\mathrm{m}, 3 \mathrm{H}), 4.22-4.40(\mathrm{~m}, 2 \mathrm{H})$ and $5.19(\mathrm{~d}, J=5.6 \mathrm{~Hz}, 1 \mathrm{H})$.

\section{$1^{\prime}$-N-(tert-butoxycarbonyl)-1'-demethyl-4' -depropyl-4' -n-pentyl- 2,3,4-tris-O-(trimethylsilyl)lincomycin (26)}

Compound 21 (crude), trimethylchlorosilane $(2.6 \mathrm{ml}, 20.3 \mathrm{mmol})$ and hexamethyldisilazane $(4.24 \mathrm{ml}, 20.3 \mathrm{mmol})$ in pyridine $(10.0 \mathrm{ml})$ were treated for $1 \mathrm{~h}$ according to the similar procedure as described for the preparation of $\mathbf{2 3}$, and then the crude compound and $6 \mathrm{~N}$ acetic acid $(1.21 \mathrm{ml})$ in methanol $(23 \mathrm{ml})$ were treated for $2.0 \mathrm{~h}$ according to the similar procedure as described for the preparation of $\mathbf{2 3}$ to afford $\mathbf{2 6}(2.21 \mathrm{~g}, 74 \%$ in three steps from 14) as a colorless solid. FAB-MS $m / z 737(\mathrm{M}+\mathrm{H})^{+}$as $\mathrm{C}_{33} \mathrm{H}_{68} \mathrm{~N}_{2} \mathrm{O}_{8} \mathrm{SSi}_{3} ;{ }^{1} \mathrm{H}$ NMR $\left(400 \mathrm{MHz}, \mathrm{CDCl}_{3}\right) \delta$ 0.07-0.25 (m, 27H), 0.78-0.97 (m, 3H), 1.05-1.42 (m, $11 \mathrm{H}), 1.48(\mathrm{~s}, 9 \mathrm{H}), 2.07(\mathrm{~s}, 3 \mathrm{H}), 2.10-3.20(\mathrm{~m}, 4 \mathrm{H}), 3.40-3.90(\mathrm{~m}, 3 \mathrm{H})$, $3.92-4.20(\mathrm{~m}, 3 \mathrm{H}), 4.23-4.47(\mathrm{~m}, 2 \mathrm{H})$ and $5.19(\mathrm{br} \mathrm{d}, J=5.4 \mathrm{~Hz}, 1 \mathrm{H})$.

$1^{\prime}$-N-(tert-butoxycarbonyl)-1'-demethyl-4'-depropyl-4' - $\{(E)$-pent-2enyl\}-2,3,4-tris-O-(trimethylsilyl)lincomycin (27)

Compound 22 (crude), trimethylchlorosilane $(3.50 \mathrm{ml}, 27.4 \mathrm{mmol})$ and hexamethyldisilazane $(5.70 \mathrm{ml}, 27.4 \mathrm{mmol})$ in pyridine $(10 \mathrm{ml})$ were treated for $1 \mathrm{~h}$ according to the similar procedure as described for the preparation of $\mathbf{2 3}$ and then, the crude compound and $6 \mathrm{~N}$ acetic acid $(1.64 \mathrm{ml})$ in methanol $(31 \mathrm{ml})$ were treated for $1.0 \mathrm{~h}$ according to the similar procedure as described for the preparation of $\mathbf{2 3}$ to afford $\mathbf{2 7}$ (3.29 g, 81.8\% in three steps from 15) as a colorless solid. ${ }^{1} \mathrm{H}$ NMR $\left(400 \mathrm{MHz}, \mathrm{CDCl}_{3}\right) \delta 0.02-0.28(\mathrm{~m}, 27 \mathrm{H}), 0.96$ (t, $J=7.4 \mathrm{~Hz}, 3 \mathrm{H}), 1.08-1.25(\mathrm{~m}, 3 \mathrm{H}), 1.48(\mathrm{~s}, 9 \mathrm{H}), 1.89-2.51(\mathrm{~m}, 6 \mathrm{H}), 2.02$ (s, 3H), 2.66-3.26 (m, 2H), 3.40-3.64 (m, 2H), 3.68-4.19 (m, 4H), 4.22-4.50 $(\mathrm{m}, 2 \mathrm{H}), 5.19(\mathrm{br} \mathrm{d}, J=5.1 \mathrm{~Hz}, 1 \mathrm{H}), 5.26-5.39(\mathrm{~m}, 1 \mathrm{H})$ and $5.43-5.59(\mathrm{~m}, 1 \mathrm{H})$.

\section{$7(S)-1^{\prime}-N$-(tert-butoxycarbonyl)-1' -demethyl-7-deoxy-7-[5-\{5- (methylamino)thiazol-4-yl\}-1,3,4-thiadiazol-2-ylthio]-2,3,4-tris-O- (trimethylsilyl)lincomycin (31)}

To a solution of compound $23(200 \mathrm{mg}, 0.28 \mathrm{mmol})$ in THF $(2 \mathrm{ml})$ at $0{ }^{\circ} \mathrm{C}$ were added triphenylphosphine $(110.9 \mathrm{mg}, 0.42 \mathrm{mmol})$, diethylazodicarboxylate $(77 \mu \mathrm{l}, 0.42 \mathrm{mmol}), 5$ - $\{5$-(methylamino)thiazol-4-yl $\}-1,3,4$-thiadiazole-2thiol $(100.7 \mathrm{mg}, 0.44 \mathrm{mmol})$ and stirred at room temperature for $18 \mathrm{~h}$. The solution was purified by preparative TLC (hexane/ethyl acetate $=2 / 1$ ) to obtain the title compound as an off-white solid (88.8 mg, 34.2\%). FAB-MS $\mathrm{m} / z 921$ $(\mathrm{M}+\mathrm{H})^{+}$as $\mathrm{C}_{37} \mathrm{H}_{68} \mathrm{~N}_{6} \mathrm{O}_{7} \mathrm{~S}_{4} \mathrm{Si}_{3} ;{ }^{1} \mathrm{H}$ NMR $\left(400 \mathrm{MHz}, \mathrm{CDCl}_{3}\right) \delta \quad 0.00-0.25$ $(\mathrm{m}, 27 \mathrm{H}), \quad 0.80-1.00(\mathrm{~m}, 3 \mathrm{H}), 1.08-1.67(\mathrm{~m}, 16 \mathrm{H}), 1.74-2.99(\mathrm{~m}, 7 \mathrm{H})$, 3.02-3.22 (m, 3H), 3.43-3.90 (m, 3H), 3.98-4.50 (m, 4H), 4.60-4.94 $(\mathrm{m}, 1 \mathrm{H}), 5.20(\mathrm{br} \mathrm{d}, J=5.4 \mathrm{~Hz}, 1 \mathrm{H})$ and $7.85-8.00$ (br s, $1 \mathrm{H})$.

\section{7(S)-7-(6-aminobenzothiazol-2-ylthio)-7-deoxy-1'-} demethyllincomycin (32)

To a solution of compound $23(200 \mathrm{mg}, 0.28 \mathrm{mmol})$ in THF $(2 \mathrm{ml})$ at $0{ }^{\circ} \mathrm{C}$ were added triphenylphosphine (110.9 mg, $0.42 \mathrm{mmol})$, diethylazodicarboxylate $(77 \mu \mathrm{l}, 0.42 \mathrm{mmol}), 6$-aminobenzothiazole-2-thiol $(79.7 \mathrm{mg}, 0.44 \mathrm{mmol})$ and stirred at room temperature for $4 \mathrm{~h}$. To the solution was added $1 \mathrm{~N} \mathrm{HCl}$ $(1 \mathrm{ml})-\mathrm{MeOH}(1 \mathrm{ml})$ and stirred at room temperature for $30 \mathrm{~min}$. The solution was added to the saturated aqueous $\mathrm{NaHCO}_{3}$. The desired compound was extracted with ethyl acetate, and then the organic phase was dried over $\mathrm{Na}_{2} \mathrm{SO}_{4}$, filtrated and concentrated under reduced pressure. The resulting residue was purified by preparative TLC $\left(\mathrm{CHCl}_{3} / \mathrm{MeOH} / 28 \%\right.$ aq. $\left.\mathrm{NH}_{4} \mathrm{OH}=9 / 2 / 0.2\right)$ to obtain 7(S)-7-(6-aminobenzothiazol-2-yl)thio-1'-N-(tert-butoxycarbonyl)-1'demethyl-7-deoxy-2,3,4-tris-O-(trimethylsilyl)lincomycin (197.2 mg, crude).

To the solution of this intermediate in $\mathrm{MeOH}(2 \mathrm{ml})$ was added $4 \mathrm{~N} \mathrm{HCl}-$ ethyl acetate $(2.5 \mathrm{ml})$ and stirred at room temperature for $2 \mathrm{~h}$. The solution was concentrated under reduced pressure. The resulting residue was purified by silica gel column chromatography $\left(\mathrm{CHCl}_{3} / \mathrm{MeOH} / 28 \%\right.$ aq. $\left.\mathrm{NH}_{4} \mathrm{OH}=9 / 2 / 0.2\right)$ to obtain the title compound $(96.4 \mathrm{mg}, 61.4 \%$ in three steps from 23 ) as an offwhite solid. $[\alpha]_{\mathrm{D}}{ }^{26}+92.1^{\circ}$ (c 2.49, MeOH); ESI-MS $m / z 557(\mathrm{M}+\mathrm{H})^{+}$as $\mathrm{C}_{24} \mathrm{H}_{36} \mathrm{~N}_{4} \mathrm{O}_{5} \mathrm{~S}_{3}$; TOF-ESI-HRMS $(\mathrm{M}+\mathrm{H})^{+}$calcd. for $\mathrm{C}_{24} \mathrm{H}_{36} \mathrm{~N}_{4} \mathrm{O}_{5} \mathrm{~S}_{3}: 557.1926$, found: 557.1920; ${ }^{1} \mathrm{H}$ NMR $\left(400 \mathrm{MHz}, \mathrm{CD}_{3} \mathrm{OD}\right) \delta 0.86-0.96(\mathrm{~m}, 3 \mathrm{H}), 1.25-1.40$ $(\mathrm{m}, 4 \mathrm{H}), 1.49(\mathrm{~d}, J=6.9 \mathrm{~Hz}, 3 \mathrm{H}), 1.69-1.82(\mathrm{~m}, 1 \mathrm{H}), 1.93(\mathrm{~s}, 3 \mathrm{H}), 1.96-2.13$ (m, $2 \mathrm{H}), 2.52(\mathrm{dd}, J=10.4,8.1 \mathrm{~Hz}, 1 \mathrm{H}), 3.20(\mathrm{dd}, J=10.4,6.9 \mathrm{~Hz}, 1 \mathrm{H}), 3.58$ (dd, $J=10.2,3.2 \mathrm{~Hz}, 1 \mathrm{H}), 3.80-3.87(\mathrm{~m}, 2 \mathrm{H}), 4.11(\mathrm{dd}, J=10.2,5.6 \mathrm{~Hz}, 1 \mathrm{H})$, $4.27(\mathrm{dq}, J=6.9,2.7 \mathrm{~Hz}, 1 \mathrm{H}), 4.39(\mathrm{br} \mathrm{dd}, J=10.0,0.9 \mathrm{~Hz}, 1 \mathrm{H}), 4.57$ (dd, $J=10.0,2.7 \mathrm{~Hz}, 1 \mathrm{H}), 5.26(\mathrm{~d}, J=5.6 \mathrm{~Hz}, 1 \mathrm{H}), 6.85(\mathrm{dd}, J=8.7,2.1 \mathrm{~Hz}$, $1 \mathrm{H}), 7.08(\mathrm{~d}, J=2.1 \mathrm{~Hz}, 1 \mathrm{H})$ and $7.59(\mathrm{~d}, J=8.7 \mathrm{~Hz}, 1 \mathrm{H})$.

\section{7(S)-7-(5-amino-1,3,4-thiadiazol-2-ylthio)-7-deoxy-1'-} demethyllincomycin (33)

To a solution of compound $23(200 \mathrm{mg}, 0.28 \mathrm{mmol})$ in THF $(2 \mathrm{ml})$ at $0{ }^{\circ} \mathrm{C}$ were added triphenylphosphine $(138.0 \mathrm{mg}, 0.53 \mathrm{mmol})$, diethylazodicarboxylate $(96 \mu \mathrm{l}, 0.53 \mathrm{mmol}), 5$-(tert-butoxycarbonylamino)-1,3,4-thiadiazole-2-thiol $(126.9 \mathrm{mg}, 0.54 \mathrm{mmol})$ and stirred at room temperature for $3 \mathrm{~h}$. To the solution was added $1 \mathrm{~N} \mathrm{HCl}(1 \mathrm{ml})-\mathrm{MeOH}(1 \mathrm{ml})$, and stirred at room temperature for $50 \mathrm{~min}$. The solution was added to the saturated aqueous $\mathrm{NaHCO}_{3}$. The desired compound was extracted with ethyl acetate, and then the organic phase was dried over $\mathrm{Na}_{2} \mathrm{SO}_{4}$, filtrated and concentrated under reduced pressure. The resulting residue was purified by preparative TLC $\left(\mathrm{CHCl}_{3} /\right.$ $\mathrm{MeOH} / 28 \%$ aq. $\left.\mathrm{NH}_{4} \mathrm{OH}=9 / 2 / 0.2\right)$ to obtain $7(S)-7-[5-\{($ tert-butoxycarbonyl $)$ amino\}-1,3,4-thiadiazol-2-ylthio]-1'-N-(tert-butoxycarbonyl)-1'-demethyl-7deoxylincomycin as a colorless solid (146.6 mg, 73.4\%). ${ }^{1} \mathrm{H}$ NMR $(400 \mathrm{MHz}$, $\left.\mathrm{CD}_{3} \mathrm{OD}\right) \delta 0.85-0.98(\mathrm{~m}, 3 \mathrm{H}), 1.25-1.60(\mathrm{~m}, 25 \mathrm{H}), 1.80-1.97(\mathrm{~m}, 1 \mathrm{H})$, $1.98-2.17(\mathrm{~m}, 1 \mathrm{H}), 2.06,2.10$ ( $\mathrm{s} \times 2,3 \mathrm{H}), 2.22-2.40(\mathrm{~m}, 1 \mathrm{H}), 2.89-3.05$ (m, $1 \mathrm{H}), 3.50-3.60(\mathrm{~m}, 1 \mathrm{H}), 3.69-3.82(\mathrm{~m}, 1 \mathrm{H}), 3.84-4.00(\mathrm{~m}, 1 \mathrm{H}), 4.02-4.20$ $(\mathrm{m}, 2 \mathrm{H}), 4.26-4.42(\mathrm{~m}, 2 \mathrm{H}), 4.45-4.60(\mathrm{~m}, 1 \mathrm{H})$ and $5.27(\mathrm{br} \mathrm{d}, J=5.4 \mathrm{~Hz}, 1 \mathrm{H})$.

To the solution of this intermediate $(146.6 \mathrm{mg}, 0.21 \mathrm{mmol})$ in $\mathrm{MeOH}$ $(1.4 \mathrm{ml})$ was added $4 \mathrm{~N} \mathrm{HCl}$-ethyl acetate $(1.7 \mathrm{ml})$, and stirred at room temperature for $2 \mathrm{~h}$. The solution was concentrated under reduced pressure. The resulting residue was purified by preparative TLC $\left(\mathrm{CHCl}_{3} / \mathrm{MeOH} / 28 \%\right.$ aq. $\left.\mathrm{NH}_{4} \mathrm{OH}=9 / 2 / 0.2\right)$ to obtain the title compound $(25.4 \mathrm{mg}, 24.2 \%)$ as a colorless solid. $[\alpha]_{\mathrm{D}}^{25}+101^{\circ}(c 0.33, \mathrm{MeOH})$; ESI-MS $m / z 508(\mathrm{M}+\mathrm{H})^{+}$as $\mathrm{C}_{19} \mathrm{H}_{33} \mathrm{~N}_{5} \mathrm{O}_{5} \mathrm{~S}_{3}$; TOF-ESI-HRMS $(\mathrm{M}+\mathrm{H})^{+}$calcd. for $\mathrm{C}_{19} \mathrm{H}_{33} \mathrm{~N}_{5} \mathrm{O}_{5} \mathrm{~S}_{3}: 508.1722$, found: $508.1719 ;{ }^{1} \mathrm{H}$ NMR $\left(400 \mathrm{MHz}, \mathrm{CD}_{3} \mathrm{OD}\right) \delta 0.89-0.98(\mathrm{~m}, 3 \mathrm{H}), 1.33-1.45$ $(\mathrm{m}, 4 \mathrm{H}), 1.37(\mathrm{~d}, J=7.0 \mathrm{~Hz}, 3 \mathrm{H}), 1.84-1.95(\mathrm{~m}, 1 \mathrm{H}), 2.03-2.18(\mathrm{~m}, 2 \mathrm{H}), 2.11$ (s, 3H), 2.66 (dd, $J=10.6,8.3 \mathrm{~Hz}, 1 \mathrm{H}), 3.30-3.36(\mathrm{~m}, 1 \mathrm{H}), 3.56$ (dd, $J=10.2$, $3.2 \mathrm{~Hz}, 1 \mathrm{H}), 3.81$ (br dd, $J=3.2,0.7 \mathrm{~Hz}, 1 \mathrm{H}), 3.96(\mathrm{dq}, J=7.0,2.6 \mathrm{~Hz}, 1 \mathrm{H})$, $4.00(\mathrm{dd}, J=9.3,4.0 \mathrm{~Hz}, 1 \mathrm{H}), 4.09$ (dd, $J=10.2,5.6 \mathrm{~Hz}, 1 \mathrm{H}), 4.37$ (br dd, $J=10.0, \quad 0.7 \mathrm{~Hz}, \quad 1 \mathrm{H}), \quad 4.52 \quad(\mathrm{dd}, \quad J=10.0, \quad 2.6 \mathrm{~Hz}, \quad 1 \mathrm{H})$ and 5.27 $(\mathrm{d}, J=5.6 \mathrm{~Hz}, 1 \mathrm{H})$.

\section{7(S)-1'-demethyl-7-deoxy-7-\{5-(2-nitrophenyl)-1,3,4-thiadiazol-2- ylthio\}lincomycin (34)}

To a solution of compound $23(200 \mathrm{mg}, 0.28 \mathrm{mmol})$ in THF $(2 \mathrm{ml})$ at $0{ }^{\circ} \mathrm{C}$ were added triphenylphosphine $(110.9 \mathrm{mg}, 0.42 \mathrm{mmol})$, diethylazodicarboxylate $(77 \mu \mathrm{l}, 0.42 \mathrm{mmol}), 5$-(2-nitrophenyl)-1,3,4-thiadiazole-2-thiol $(104.6 \mathrm{mg}$, $0.44 \mathrm{mmol}$ ), and stirred at room temperature for $7 \mathrm{~h}$. The solution was added to the saturated aqueous $\mathrm{NaHCO}_{3}$. The desired compound was extracted with ethyl acetate, and then the organic phase was dried over $\mathrm{Na}_{2} \mathrm{SO}_{4}$, filtrated and concentrated under reduced pressure. To the resulting residue was added $\mathrm{MeOH}(4 \mathrm{ml})-1 \mathrm{~N} \mathrm{HCl}(1 \mathrm{ml})$ and stirred at room temperature for $2.5 \mathrm{~h}$. The solution was added to the saturated aqueous $\mathrm{NaHCO}_{3}$. The desired compound was extracted with ethyl acetate, and then the organic phase was dried over $\mathrm{Na}_{2} \mathrm{SO}_{4}$, filtrated and concentrated under reduced pressure. The resulting residue was purified by preparative TLC $\left(\mathrm{CHCl}_{3} / \mathrm{MeOH} / 28 \%\right.$ aq. $\mathrm{NH}_{4} \mathrm{OH}=9$ /2/0.2) to obtain 7(S)-1'-N-(tert-butoxycarbonyl)-1'-demethyl-7-deoxy-7-\{5(2-nitrophenyl)-1,3,4-thiadiazol-2-ylthio\}lincomycin (164.9 mg as crude). To 
the solution of this crude compound $(63.9 \mathrm{mg})$ in $\mathrm{MeOH}(0.6 \mathrm{ml})$ was added $4 \mathrm{~N} \mathrm{HCl}$-ethyl acetate $(0.75 \mathrm{ml})$, and stirred at room temperature for $2.5 \mathrm{~h}$. The solution was concentrated under reduced pressure. The resulting residue was purified by preparative TLC $\left(\mathrm{CHCl}_{3} / \mathrm{MeOH} / 28 \%\right.$ aq. $\left.\mathrm{NH}_{4} \mathrm{OH}=9 / 2 / 0.2\right)$ to obtain the title compound $(13.0 \mathrm{mg}, 19.4 \%$ in three steps from 23 ) as a colorless solid. $[\alpha]_{\mathrm{D}}{ }^{26}+37.1^{\circ}(c 0.21, \mathrm{MeOH})$; ESI-MS $m / z 614(\mathrm{M}+\mathrm{H})^{+}$as $\mathrm{C}_{25} \mathrm{H}_{35} \mathrm{~N}_{5} \mathrm{O}_{7} \mathrm{~S}_{3}$; TOF-ESI-HRMS $(\mathrm{M}+\mathrm{H})^{+}$calcd. for $\mathrm{C}_{25} \mathrm{H}_{35} \mathrm{~N}_{5} \mathrm{O}_{7} \mathrm{~S}_{3}$ : 614.1777, found: 614.1778; ${ }^{1} \mathrm{H}$ NMR (400 MHz, $\left.\mathrm{CD}_{3} \mathrm{OD}\right) \delta 0.83-0.99(\mathrm{~m}, 3 \mathrm{H}), 1.30-1.45$ $(\mathrm{m}, 4 \mathrm{H}), 1.56(\mathrm{~d}, J=7.0 \mathrm{~Hz}, 3 \mathrm{H}), 1.88-1.98(\mathrm{~m}, 1 \mathrm{H}), 1.99(\mathrm{~s}, 3 \mathrm{H}), 2.07-2.25$ $(\mathrm{m}, 2 \mathrm{H}), 2.69$ (br dd, $J=10.6,8.4 \mathrm{~Hz}, 1 \mathrm{H}), 3.32-3.42(\mathrm{~m}, 1 \mathrm{H}), 3.56$ (dd, $J=10.2,3.2 \mathrm{~Hz}, 1 \mathrm{H}), 3.82-3.88(\mathrm{~m}, 1 \mathrm{H}), 4.00-4.10(\mathrm{~m}, 1 \mathrm{H}), 4.10$ (dd, $J=10.2,5.6 \mathrm{~Hz}, 1 \mathrm{H}), 4.36-4.46(\mathrm{~m}, 1 \mathrm{H}), 4.47(\mathrm{dq}, J=7.0,2.5 \mathrm{~Hz}, 1 \mathrm{H})$, $4.66(\mathrm{dd}, J=10.0,2.5 \mathrm{~Hz}, 1 \mathrm{H}), 5.28(\mathrm{~d}, J=5.6 \mathrm{~Hz}, 1 \mathrm{H}), 7.74-7.87(\mathrm{~m}, 3 \mathrm{H})$ and $8.05-8.15(\mathrm{~m}, 1 \mathrm{H})$.

7(S)-1'-demethyl-7-deoxy-7-[5-\{5-(methylamino)thiazol-4-yl\}-1,3,4thiadiazol-2-ylthio]lincomycin (35)

To the solution of compound 31 ( $88.8 \mathrm{mg} 0.096 \mathrm{mmol})$ in $\mathrm{MeOH}(0.5 \mathrm{ml})$ was added $4 \mathrm{~N} \mathrm{HCl}$-ethyl acetate $(0.79 \mathrm{ml})$, stirred at $0{ }^{\circ} \mathrm{C}$ for $1 \mathrm{~h}$ and then stirred at room temperature for $3.5 \mathrm{~h}$. The solution was concentrated under reduced pressure. The resulting residue was purified by preparative TLC $\left(\mathrm{CHCl}_{3} /\right.$ $\mathrm{MeOH} / 28 \%$ aq. $\left.\mathrm{NH}_{4} \mathrm{OH}=9 / 2 / 0.2\right)$ to obtain the title compound $(39.8 \mathrm{mg}$, $68.3 \%)$ as an off-white solid. $[\alpha]_{\mathrm{D}}{ }^{26}+69.5^{\circ}(c 0.60, \mathrm{MeOH})$; ESI-MS $m / z 605$ $(\mathrm{M}+\mathrm{H})^{+} \quad$ as $\quad \mathrm{C}_{23} \mathrm{H}_{36} \mathrm{~N}_{6} \mathrm{O}_{5} \mathrm{~S}_{4}$; TOF-ESI-HRMS $\quad(\mathrm{M}+\mathrm{H})^{+}$calcd. for $\mathrm{C}_{23} \mathrm{H}_{36} \mathrm{~N}_{6} \mathrm{O}_{5} \mathrm{~S}_{4}$ : 605.1708, found: 605.1706; ${ }^{1} \mathrm{H}$ NMR (400 MHz, $\left.\mathrm{CD}_{3} \mathrm{OD}\right)$ $\delta$ 0.86-0.95 (m, 3H), $1.29-1.41(\mathrm{~m}, 4 \mathrm{H}), 1.49(\mathrm{~d}, J=6.9 \mathrm{~Hz}, 3 \mathrm{H}), 1.78-1.87$ (m, 1H), 1.98-2.14 (m, 2H), $2.01(\mathrm{~s}, 3 \mathrm{H}), 2.56(\mathrm{dd}, J=10.5,8.1 \mathrm{~Hz}, 1 \mathrm{H}), 3.11$ (s, $3 \mathrm{H}), 3.25(\mathrm{dd}, J=10.5,7.0 \mathrm{~Hz}, 1 \mathrm{H}), 3.56(\mathrm{dd}, J=10.3,3.2 \mathrm{~Hz}, 1 \mathrm{H}), 3.82$ (br dd, $J=3.2,0.9 \mathrm{~Hz}, 1 \mathrm{H}), 3.86$ (dd, $J=9.2,3.9 \mathrm{~Hz}, 1 \mathrm{H}), 4.09$ (dd, $J=10.3$, $5.6 \mathrm{~Hz}, 1 \mathrm{H}), 4.27(\mathrm{dq}, J=6.9,2.7 \mathrm{~Hz}, 1 \mathrm{H}), 4.39$ (br dd, $J=10.0,0.9 \mathrm{~Hz}, 1 \mathrm{H})$, $4.59(\mathrm{dd}, J=10.0,2.7 \mathrm{~Hz}, 1 \mathrm{H}), 5.26(\mathrm{~d}, J=5.6 \mathrm{~Hz}, 1 \mathrm{H})$ and $8.12(\mathrm{~s}, 1 \mathrm{H})$.

7(S)-7-deoxy-7-[5-\{5-(methylamino)thiazol-4-yl\}-1,3,4-thiadiazol-2ylthio]lincomycin (37)

Compound 36 (240 mg, $0.39 \mathrm{mmol})$, triphenylphosphine $(150.0 \mathrm{mg}$, $0.57 \mathrm{mmol}$ ), diethylazodicarboxylate $(100 \mu \mathrm{l}, 0.64 \mathrm{mmol})$ and 5-\{5-(methylamino)thiazol-4-yl $\}-1,3,4$-thiadiazole-2-thiol $(150.0 \mathrm{mg}, 0.65 \mathrm{mmol})$ in THF $(5 \mathrm{ml})$ were treated for $2 \mathrm{~h}$, and then to the solution was added $1 \mathrm{~N} \mathrm{HCl}$ $(0.5 \mathrm{ml})-\mathrm{MeOH}(5 \mathrm{ml})$ and stirred at room temperature for $1 \mathrm{~h}$. The solution was then added to the saturated aqueous $\mathrm{NaHCO}_{3}$. The desired compound was extracted with ethyl acetate, and then the organic phase was dried over $\mathrm{Na}_{2} \mathrm{SO}_{4}$, filtrated and concentrated under reduced pressure. The resulting residue was purified by preparative TLC $\left(\mathrm{CHCl}_{3} / \mathrm{MeOH} / 28 \%\right.$ aq. $\left.\mathrm{NH}_{4} \mathrm{OH}=9 / 2 / 0.2\right)$ to obtain the title compound 37 (88.2 mg, $37 \%$ in two steps) as an off-white solid. $[\alpha]_{D}{ }^{26}+125^{\circ}(c \quad 0.89, \mathrm{MeOH})$; ESI-MS $m / z 619(\mathrm{M}+\mathrm{H})^{+}$as $\mathrm{C}_{24} \mathrm{H}_{38} \mathrm{~N}_{6} \mathrm{O}_{5} \mathrm{~S}_{4}$; TOF-ESI-HRMS $(\mathrm{M}+\mathrm{H})^{+}$calcd. for $\mathrm{C}_{24} \mathrm{H}_{38} \mathrm{~N}_{6} \mathrm{O}_{5} \mathrm{~S}_{4}: 619.1865$, found: 619.1860; ${ }^{1} \mathrm{H}$ NMR $\left(400 \mathrm{MHz}, \mathrm{CD}_{3} \mathrm{OD}\right) \delta$ 0.82-0.98 (m, 3H), 1.21-1.39 (m, 4H), 1.53 $(\mathrm{d}, J=6.9 \mathrm{~Hz}, 3 \mathrm{H}), 1.78-1.89(\mathrm{~m}, 1 \mathrm{H}), 1.92-2.08(\mathrm{~m}, 2 \mathrm{H}), 2.02(\mathrm{~s}, 3 \mathrm{H})$, 2.09-2.25 (m, 1H), 2.35 (s, 3H), $3.00(\mathrm{dd}, J=10.5,5.0 \mathrm{~Hz}, 1 \mathrm{H}), 3.13(\mathrm{~s}, 3 \mathrm{H})$, $3.19(\mathrm{dd}, J=8.5,6.2 \mathrm{~Hz}, 1 \mathrm{H}), 3.59(\mathrm{dd}, J=10.2,3.2 \mathrm{~Hz}, 1 \mathrm{H}), 3.77-3.85$ (m, 1H), 4.11 (dd, $J=10.2,5.6 \mathrm{~Hz}, 1 \mathrm{H}), 4.27$ (dq, $J=6.9,3.0 \mathrm{~Hz}, 1 \mathrm{H}), 4.44$ (br dd, $J=9.8,0.5 \mathrm{~Hz}, 1 \mathrm{H}), 4.57(\mathrm{dd}, J=9.8,3.0 \mathrm{~Hz}, 1 \mathrm{H}), 5.27(\mathrm{~d}, J=5.6 \mathrm{~Hz}$, $1 \mathrm{H})$ and $8.13(\mathrm{~s}, 1 \mathrm{H})$.

\section{7(S)-1'-demethyl-7-deoxy-7-\{5-(2-nitrophenyl)-1,3,4-thiadiazol-2-} yl thio-1'- $\mathrm{N}$-i-propyllincomycin (38)

To a solution of compound $34(30.5 \mathrm{mg}, 0.05 \mathrm{mmol})$ in 1,2-dichloroethane $(1 \mathrm{ml})$ at $0{ }^{\circ} \mathrm{C}$ were added acetone $(40 \mu \mathrm{l}, 0.054 \mathrm{mmol}), \mathrm{AcOH}$ (one drop) and $\mathrm{NaBH}(\mathrm{OAc})_{3}(21.7 \mathrm{mg}, 0.10 \mathrm{mmol})$ and stirred at room temperature for $15 \mathrm{~h}$. The mixture was concentrated under reduced pressure. The resulting residue was purified by preparative TLC $\left(\mathrm{CHCl}_{3} / \mathrm{MeOH} / 28 \%\right.$ aq $\left.\mathrm{NH}_{4} \mathrm{OH}=10 / 1 / 0.1\right)$ and then by $\mathrm{LH}-20\left(\mathrm{CHCl}_{3} / \mathrm{MeOH}=1 / 1\right)$ to obtain the title compound $(20.1 \mathrm{mg}, 61 \%)$ as a colorless solid. $[\alpha]_{\mathrm{D}}{ }^{26}+76.8^{\circ}(c 0.59, \mathrm{MeOH})$; ESI-MS $\mathrm{m} / \mathrm{z} 656(\mathrm{M}+\mathrm{H})^{+}$as $\mathrm{C}_{28} \mathrm{H}_{41} \mathrm{~N}_{5} \mathrm{O}_{7} \mathrm{~S}_{3}$; TOF-ESI-HRMS $(\mathrm{M}+\mathrm{H})^{+}$calcd. for $\mathrm{C}_{28} \mathrm{H}_{41} \mathrm{~N}_{5} \mathrm{O}_{7} \mathrm{~S}_{3}: 656.2246$, found: 656.2243; ${ }^{1} \mathrm{H}$ NMR (400 MHz, $\left.\mathrm{CD}_{3} \mathrm{OD}\right)$ $\delta \quad 0.86-0.98(\mathrm{~m}, 3 \mathrm{H}), \quad 1.06-1.16(\mathrm{~m}, 6 \mathrm{H}), \quad 1.28-1.42(\mathrm{~m}, 4 \mathrm{H}), \quad 1.58$ $(\mathrm{d}, J=6.9 \mathrm{~Hz}, 3 \mathrm{H}), 1.69-1.81(\mathrm{~m}, 1 \mathrm{H}), 1.99(\mathrm{~s}, 3 \mathrm{H}), 1.97-2.07(\mathrm{~m}, 1 \mathrm{H})$, 2.07-2.20 (m, 1H), 2.20-2.31 (m, 1H), 2.76-2.90 (m, 1H), 3.26-3.34 (m, 1H), $3.39-3.52(\mathrm{~m}, 1 \mathrm{H}), 3.57(\mathrm{dd}, J=10.2,3.2 \mathrm{~Hz}, 1 \mathrm{H}), 3.82(\mathrm{br} \mathrm{dd}, J=3.2,0.8 \mathrm{~Hz}$, $1 \mathrm{H}), 4.10(\mathrm{dd}, J=10.2,5.6 \mathrm{~Hz}, 1 \mathrm{H}), 4.40($ br dd, $J=9.3,0.8 \mathrm{~Hz}, 1 \mathrm{H}), 4.51$ $(\mathrm{dq}, J=6.9,3.4 \mathrm{~Hz}, 1 \mathrm{H}), 4.59(\mathrm{dd}, J=9.3,3.4 \mathrm{~Hz}, 1 \mathrm{H}), 5.26(\mathrm{~d}, J=5.6 \mathrm{~Hz}, 1 \mathrm{H})$, 7.74-7.86 $(\mathrm{m}, 3 \mathrm{H})$ and $8.06-8.12(\mathrm{~m}, 1 \mathrm{H})$.

$7(S)-1^{\prime}-N-\{2$-(tert-butyldimethylsilyloxy)ethyl $\}-1^{\prime}$-demethyl-7-deoxy7-\{5-(2-nitrophenyl)-1,3,4-thiadiazol-2-ylthio\}lincomycin (39)

Compound 34 (49.2 mg, $0.08 \mathrm{mmol}), 2$-(tert-butyldimethylsilyloxy)acetaldehyde $\left(23 \mu \mathrm{l}, 0.12 \mathrm{mmol}\right.$ ), $\mathrm{AcOH}$ (one drop) and $\mathrm{NaBH}(\mathrm{OAc})_{3}(34.2 \mathrm{mg}$, $0.16 \mathrm{mmol})$ in 1,2-dichloroethane $(1 \mathrm{ml})$ were treated at $0{ }^{\circ} \mathrm{C}$ for $15 \mathrm{~h}$ according to the similar procedure as described for the preparation of $\mathbf{3 8}$ to afford $39(26.9 \mathrm{mg}, 44.0 \%)$ as a colorless solid. FAB-MS $\mathrm{m} / \mathrm{z} 772(\mathrm{M}+\mathrm{H})^{+}$as $\mathrm{C}_{33} \mathrm{H}_{53} \mathrm{~N}_{5} \mathrm{O}_{8} \mathrm{~S}_{3} \mathrm{Si} ;{ }^{1} \mathrm{H}$ NMR $\left(400 \mathrm{MHz}, \mathrm{CD}_{3} \mathrm{OD}\right) \delta 0.07$ (s, 3H), $0.08(\mathrm{~s}, 3 \mathrm{H})$, $0.82-0.98(\mathrm{~m}, 12 \mathrm{H}), 1.25-1.42(\mathrm{~m}, 4 \mathrm{H}), 1.59(\mathrm{~d}, J=6.8 \mathrm{~Hz}, 1 \mathrm{H}), 1.68-1.87$ $(\mathrm{m}, 1 \mathrm{H}), 1.94-2.05(\mathrm{~m}, 1 \mathrm{H}), 1.97(\mathrm{~s}, 3 \mathrm{H}), 2.07-2.21(\mathrm{~m}, 2 \mathrm{H}), 2.54-2.92$ $(\mathrm{m}, 2 \mathrm{H}), 3.37-3.44(\mathrm{~m}, 1 \mathrm{H}), 3.56(\mathrm{dd}, J=10.2,3.2 \mathrm{~Hz}, 1 \mathrm{H}), 3.71-3.92(\mathrm{~m}, 4 \mathrm{H})$, $4.10(\mathrm{dd}, J=10.2,5.6 \mathrm{~Hz}, 1 \mathrm{H}), 4.35-4.44(\mathrm{~m}, 1 \mathrm{H}), 4.47-4.56(\mathrm{~m}, 1 \mathrm{H})$, $4.58-4.65(\mathrm{~m}, 1 \mathrm{H}), 5.26(\mathrm{~d}, J=5.6 \mathrm{~Hz}, 1 \mathrm{H}), 7.74-7.86(\mathrm{~m}, 3 \mathrm{H})$ and 8.06-8.13 (m, 1H).

\section{7(S)-1'-demethyl-7-deoxy-1'-N-(2-hydroxyethyl)-7-\{5-(2-} nitrophenyl)-1,3,4-thiadiazol-2-ylthio $\}$ lincomycin (40)

To a solution of compound $39(26.9 \mathrm{mg}, 0.035 \mathrm{mmol})$ in THF $(0.5 \mathrm{ml})$ at $0{ }^{\circ} \mathrm{C}$ were added $1 \mathrm{M}$ THF solution of tetra- $n$-butyl ammonium fluoride $(100 \mu \mathrm{l}$, $0.10 \mathrm{mmol}$ ) and stirred at room temperature for $15 \mathrm{~h}$. The mixture was concentrated under reduced pressure. The resulting residue was purified by preparative TLC $\left(\mathrm{CHCl}_{3} / \mathrm{MeOH} / 28 \%\right.$ aq $\left.\mathrm{NH}_{4} \mathrm{OH}=10 / 1 / 0.1\right)$ to obtain the title compound $(16.5 \mathrm{mg}, 72 \%)$ as a colorless solid. $[\alpha]_{\mathrm{D}}{ }^{25}+70.5^{\circ}(c 0.22, \mathrm{MeOH})$; ESI-MS $m / z 658(\mathrm{M}+\mathrm{H})^{+}$as $\mathrm{C}_{27} \mathrm{H}_{39} \mathrm{~N}_{5} \mathrm{O}_{8} \mathrm{~S}_{3}$; TOF-ESI-HRMS $(\mathrm{M}+\mathrm{H})^{+}$calcd. for $\mathrm{C}_{27} \mathrm{H}_{39} \mathrm{~N}_{5} \mathrm{O}_{8} \mathrm{~S}_{3}$ : 658.2039, found: 658.2044; ${ }^{1} \mathrm{H}$ NMR $\left(400 \mathrm{MHz}, \mathrm{CD}_{3} \mathrm{OD}\right)$

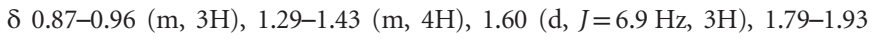
(m, 1H), $1.99(\mathrm{~s}, 3 \mathrm{H}), 2.02-2.10(\mathrm{~m}, 1 \mathrm{H}), 2.10-2.23(\mathrm{~m}, 2 \mathrm{H}), 2.64-2.75$ (m, 1H), 2.83-2.96 (m, 1H), 3.31-3.40 (m, 1H), 3.40-3.50 (m, 1H), 3.56 (dd, $J=10.3,3.2 \mathrm{~Hz}, 1 \mathrm{H}), 3.63-3.77(\mathrm{~m}, 2 \mathrm{H}), 3.83(\mathrm{dd}, J=3.2,0.8 \mathrm{~Hz}, 1 \mathrm{H})$, 4.10 (dd, $J=10.3,5.6 \mathrm{~Hz}, 1 \mathrm{H}), 4.45$ (br dd, $J=9.9,0.8 \mathrm{~Hz}, 1 \mathrm{H}), 4.51$ (dq, $J=6.9,2.9 \mathrm{~Hz}, 1 \mathrm{H}), 4.63(\mathrm{dd}, J=9.9,2.9 \mathrm{~Hz}, 1 \mathrm{H}), 5.26(\mathrm{~d}, J=5.6 \mathrm{~Hz}, 1 \mathrm{H})$, 7.74-7.86 (m, 3H) and 8.06-8.12 (m, 1H).

\section{$7(S)-1^{\prime}-N-\left\{2-(\right.$ tert-butyldimethylsilyloxy)ethyl $\}-1^{\prime}$-demethyl-7-} deoxy-7-[5-\{5-(methylamino)thiazol-4-yl $\}-1,3,4-$ thiadiazol-2-ylthio] lincomycin (41)

Compound 35 ( $74.3 \mathrm{mg}, 0.12 \mathrm{mmol}), 2$-(tert-butyldimethylsilyloxy)acetaldehyde $\left(34 \mu \mathrm{l}, 0.18 \mathrm{mmol}\right.$ ), $\mathrm{AcOH}$ (one drop) and $\mathrm{NaBH}(\mathrm{OAc})_{3}(51.0 \mathrm{mg}$, $0.24 \mathrm{mmol})$ in 1,2-dichloroethane $(1 \mathrm{ml})$ were treated at room temperature according to the similar procedure as described for the preparation of $\mathbf{3 8}$ to afford $41(54.8 \mathrm{mg}, 60 \%)$ as a colorless solid. FAB-MS $\mathrm{m} / \mathrm{z} 763(\mathrm{M}+\mathrm{H})^{+}$as $\mathrm{C}_{31} \mathrm{H}_{54} \mathrm{~N}_{6} \mathrm{O}_{6} \mathrm{~S}_{4} \mathrm{Si} ;{ }^{1} \mathrm{H}$ NMR $\left(400 \mathrm{MHz}, \mathrm{CD}_{3} \mathrm{OD}\right) \delta 0.05$ (s, 3H), 0.06 (s, $\left.3 \mathrm{H}\right)$, $0.78-1.00(\mathrm{~m}, 12 \mathrm{H}), 1.23-1.42(\mathrm{~m}, 4 \mathrm{H}), 1.54(\mathrm{~d}, J=7.1 \mathrm{~Hz}, 3 \mathrm{H}), 1.72-1.86$ (m, 1H), 1.92-2.05 (m, 1H), 1.99 (s, 3H), 2.08-2.22 (m, 2H), 2.50-2.87 $(\mathrm{m}, 2 \mathrm{H}), 3.12(\mathrm{~s}, 3 \mathrm{H}), 3.25-3.30(\mathrm{~m}, 1 \mathrm{H}), 3.36-3.44(\mathrm{~m}, 1 \mathrm{H}), 3.52-3.60$ $(\mathrm{m}, 1 \mathrm{H}), 3.68-3.88(\mathrm{~m}, 3 \mathrm{H}), 4.10(\mathrm{dd}, J=10.2,5.6 \mathrm{~Hz}, 1 \mathrm{H}), 4.30-4.44(\mathrm{~m}, 2 \mathrm{H})$, 4.54-4.62 (m, 1H), $5.26(\mathrm{~d}, J=5.6 \mathrm{~Hz}, 1 \mathrm{H})$ and $8.13(\mathrm{~s}, 1 \mathrm{H})$.

\section{7(S)-1'-demethyl-7-deoxy-1'-N-(2-hydroxylethyl)-7-[5-\{5-} (methylamino)thiazol-4-yl\}-1,3,4-thiadiazol-2-ylthio]lincomycin (42)

Compound 41 (54.8 mg, $0.072 \mathrm{mmol}$ ) and $1 \mathrm{~m}$ THF solution of tetra- $n$-butyl ammonium fluoride $(200 \mu \mathrm{l}, 0.20 \mathrm{mmol})$ in THF $(1.0 \mathrm{ml})$ were treated at $0{ }^{\circ} \mathrm{C}$ for $1 \mathrm{~h}$ and then treated at room temperature for $5 \mathrm{~h}$ according to the similar procedure as described for the preparation of 40 to afford $\mathbf{4 2}(31.3 \mathrm{mg}, 67.0 \%)$ as a colorless solid. $[\alpha]_{D^{25}}+51.1^{\circ}(c 0.27, \mathrm{MeOH})$; ESI-MS $m / z 649(\mathrm{M}+\mathrm{H})^{+}$as $\mathrm{C}_{25} \mathrm{H}_{40} \mathrm{~N}_{6} \mathrm{O}_{6} \mathrm{~S}_{4}$; TOF-ESI-HRMS $(\mathrm{M}+\mathrm{H})^{+}$calcd. for $\mathrm{C}_{25} \mathrm{H}_{40} \mathrm{~N}_{6} \mathrm{O}_{6} \mathrm{~S}_{4}: 649.1970$, found: 649.1973; ${ }^{1} \mathrm{H}$ NMR (400 MHz, $\mathrm{CD}_{3} \mathrm{OD}$ ) $\delta$ 0.88-0.96 (m, 3H), $1.30-1.40$ 
(m, 4H), $1.54(\mathrm{~d}, J=6.9 \mathrm{~Hz}, 3 \mathrm{H}), 1.79-1.90(\mathrm{~m}, 1 \mathrm{H}), 1.97-2.08(\mathrm{~m}, 1 \mathrm{H}), 2.00$ (s, 3H), 2.09-2.21 (m, 2H), 2.62-2.72 (m, $1 \mathrm{H}), 2.83-2.93(\mathrm{~m}, 1 \mathrm{H}), 3.11$ (s, 3H), 3.30-3.37 (m, 1H), 3.39-3.45 (m, 1H), $3.56(\mathrm{dd}, J=10.2,3.2 \mathrm{~Hz}, 1 \mathrm{H})$, $3.65-3.74(\mathrm{~m}, 2 \mathrm{H}), 3.81$ (br dd, $J=3.2,0.8 \mathrm{~Hz}, 1 \mathrm{H}), 4.09$ (dd, $J=10.2,5.6 \mathrm{~Hz}$, $1 \mathrm{H}), 4.33(\mathrm{dq}, J=6.9,2.8 \mathrm{~Hz}, 1 \mathrm{H}), 4.43(\mathrm{br} \mathrm{dd}, J=9.8,0.8 \mathrm{~Hz}, 1 \mathrm{H}), 4.59$ $(\mathrm{dd}, J=9.8,2.8 \mathrm{~Hz}, 1 \mathrm{H}), 5.25(\mathrm{~d}, J=5.6 \mathrm{~Hz}, 1 \mathrm{H})$ and $8.11(\mathrm{~s}, 1 \mathrm{H})$.

7(S)-1'-demethyl-7-deoxy-1'-N-\{2(R)-hydroxypropyl $\}-7-[5-\{5-$ (methylamino)thiazol-4-yl\}-1,3,4-thiadiazol-2-ylthio]lincomycin (43) To a solution of compound $35(28.7 \mathrm{mg}, 0.048 \mathrm{mmol})$ and $N, N$-diisopropylethylamine $(10.0 \mu \mathrm{l}, 0.057 \mathrm{mmol})$ in $\mathrm{MeOH}(1 \mathrm{ml})$ at $0{ }^{\circ} \mathrm{C}$ was added $(R)-2$ methyloxirane $(0.30 \mathrm{ml}, 4.3 \mathrm{mmol})$ and stirred at $0{ }^{\circ} \mathrm{C}$ for $96 \mathrm{~h}$. The mixture was concentrated under reduced pressure. The resulting residue was purified by preparative TLC $\left(\mathrm{CHCl}_{3} / \mathrm{MeOH} / 28 \%\right.$ aq. $\left.\mathrm{NH}_{4} \mathrm{OH}=10 / 1 / 0.1\right)$ to obtain the title compound $(10.6 \mathrm{mg}, 33 \%)$ as a colorless solid. $[\alpha]_{\mathrm{D}}{ }^{26}+29.2^{\circ}(c \quad 0.15$, $\mathrm{MeOH}) ;$ ESI-MS $m / z 663(\mathrm{M}+\mathrm{H})^{+}$as $\mathrm{C}_{26} \mathrm{H}_{42} \mathrm{~N}_{6} \mathrm{O}_{6} \mathrm{~S}_{4}$; TOF-ESI-HRMS $(\mathrm{M}+\mathrm{H})$ ${ }^{+}$calcd. for $\mathrm{C}_{26} \mathrm{H}_{42} \mathrm{~N}_{6} \mathrm{O}_{6} \mathrm{~S}_{4}: 663.2127$, found: $663.2127 ;{ }^{1} \mathrm{H}$ NMR $(400 \mathrm{MHz}$, $\left.\mathrm{CD}_{3} \mathrm{OD}\right) \delta 0.87-0.96(\mathrm{~m}, 3 \mathrm{H}), 1.16(\mathrm{~d}, J=6.2 \mathrm{~Hz}, 3 \mathrm{H}), 1.27-1.42(\mathrm{~m}, 4 \mathrm{H})$, $1.55(\mathrm{~d}, J=6.9 \mathrm{~Hz}, 1 \mathrm{H}), 1.78-1.87(\mathrm{~m}, 1 \mathrm{H}), 1.98-2.27(\mathrm{~m}, 3 \mathrm{H}), 1.99(\mathrm{~s}, 3 \mathrm{H})$, 2.43-2.55 (m, 1H), 2.56-2.66 (m, 1H), $3.11(\mathrm{~s}, 3 \mathrm{H}), 3.39-3.46(\mathrm{~m}, 1 \mathrm{H}), 3.55$ (dd, $J=10.3,3.2 \mathrm{~Hz}, 1 \mathrm{H}), 3.83(\mathrm{br} \mathrm{dd}, J=3.2,0.9 \mathrm{~Hz}, 1 \mathrm{H}), 3.86-3.97(\mathrm{~m}, 1 \mathrm{H})$, $4.10(\mathrm{dd}, J=10.3,5.7 \mathrm{~Hz}, 1 \mathrm{H}), 4.33(\mathrm{dq}, J=6.9,2.9 \mathrm{~Hz}, 1 \mathrm{H}), 4.42-4.48$ $(\mathrm{m}, 1 \mathrm{H}), 4.52-4.59(\mathrm{~m}, 1 \mathrm{H}), 4.61(\mathrm{dd}, J=9.9,2.9 \mathrm{~Hz}, 1 \mathrm{H}), 5.25(\mathrm{~d}, J=5.7 \mathrm{~Hz}$, $1 \mathrm{H})$ and $8.11(\mathrm{~s}, 1 \mathrm{H})$

\section{$7(S)-1^{\prime}-N$-acetyl-1'-demethyl-7-deoxy-7-[5-\{5-(methylamino)} thiazol-4-yl\}-1,3,4-thiadiazol-2-ylthio]lincomycin (44)

To a solution of compound $35(30.2 \mathrm{mg}, 0.050 \mathrm{mmol})$ in $\mathrm{MeOH}(0.5 \mathrm{ml})$ at $0^{\circ}$ $\mathrm{C}$ was added acetic anhydride $(7.0 \mu \mathrm{l}, 0.074 \mathrm{mmol})$ and stirred at $0{ }^{\circ} \mathrm{C}$ for $1.5 \mathrm{~h}$. The mixture was concentrated under reduced pressure. The resulting residue was purified by preparative TLC $\left(\mathrm{CHCl}_{3} / \mathrm{MeOH} / 28 \%\right.$ aq. $\left.\mathrm{NH}_{4} \mathrm{OH}=10 / 1 / 0.1\right)$ to obtain the title compound $(10.8 \mathrm{mg}, 33 \%)$ as an off-white solid. $[\alpha]_{\mathrm{D}}{ }^{25}$ $+56.5^{\circ}\left(c\right.$ 1.25, MeOH); ESI-MS $m / z 647(\mathrm{M}+\mathrm{H})^{+}$as $\mathrm{C}_{25} \mathrm{H}_{38} \mathrm{~N}_{6} \mathrm{O}_{6} \mathrm{~S}_{4}$; TOF-ESIHRMS $(\mathrm{M}+\mathrm{H})^{+}$calcd. for $\mathrm{C}_{25} \mathrm{H}_{38} \mathrm{~N}_{6} \mathrm{O}_{6} \mathrm{~S}_{4}$ : 647.1814, found: 647.1807; ${ }^{1} \mathrm{H}$ NMR $\left(400 \mathrm{MHz}, \quad \mathrm{CD}_{3} \mathrm{OD}\right) \quad \delta \quad 0.87-0.98 \quad(\mathrm{~m}, \quad 3 \mathrm{H}), \quad 1.25-1.42 \quad(\mathrm{~m}, \quad 4 \mathrm{H}), \quad 1.49$ $(\mathrm{d}, J=7.0 \mathrm{~Hz}, 3 \mathrm{H}), 1.82-1.95(\mathrm{~m}, 1 \mathrm{H}), 1.98(\mathrm{~s}, 3 \mathrm{H}), 2.01-2.08(\mathrm{~m}, 1 \mathrm{H}), 2.09$ (s, 3H), 2.35-2.50 (m, 1H), $3.12(\mathrm{~s}, 3 \mathrm{H}), 3.14-3.20(\mathrm{~m}, 1 \mathrm{H}), 3.56(\mathrm{dd}, J=10.2$, $3.3 \mathrm{~Hz}, 1 \mathrm{H}), 3.79-3.87(\mathrm{~m}, 1 \mathrm{H}), 3.99(\mathrm{br} \mathrm{dd}, J=3.3,1.0 \mathrm{~Hz}, 1 \mathrm{H}), 4.09$ $(\mathrm{dd}, J=10.2,5.6 \mathrm{~Hz}, 1 \mathrm{H}), 4.27(\mathrm{dq}, J=7.0,2.4 \mathrm{~Hz}, 1 \mathrm{H}), 4.36-4.43(\mathrm{~m}, 1 \mathrm{H})$ $4.48(\mathrm{dd}, J=8.8,2.7 \mathrm{~Hz}, 1 \mathrm{H}), 4.61(\mathrm{dd}, J=10.0,2.4 \mathrm{~Hz}, 1 \mathrm{H})$ and $8.12(\mathrm{~s}, 1 \mathrm{H})$.

\section{7(S)-1'-demethyl-7-deoxy-7-[5-\{5-(methylamino)thiazol-4-yl\}-1,3,4-} thiadiazol-2-ylthio]-1'-N-(4-methylthiazol-5-ylmethyl)lincomycin (45) Compound $35 \quad(24.0 \mathrm{mg}, \quad 0.04 \mathrm{mmol}), \quad$ 4-methylthiazole-5-carbaldehyde (16.2 mg, $0.13 \mathrm{mmol}$ ), $\mathrm{AcOH}$ (one drop) and $\mathrm{NaBH}(\mathrm{OAc})_{3}(25.5 \mathrm{mg}$, $0.12 \mathrm{mmol})$ in $\mathrm{MeOH}(0.5 \mathrm{ml})$ were treated at room temperature for $15 \mathrm{~h}$ according to the similar procedure as described for the preparation of $\mathbf{3 8}$ to afford $45(6.4 \mathrm{mg}, 22.0 \%)$ as a colorless solid. $[\alpha]_{\mathrm{D}}{ }^{26}+26^{\circ}(c 0.05, \mathrm{MeOH})$; ESIMS $m / z 716(\mathrm{M}+\mathrm{H})^{+}$as $\mathrm{C}_{28} \mathrm{H}_{41} \mathrm{~N}_{7} \mathrm{O}_{5} \mathrm{~S}_{5}$; TOF-ESI-HRMS $(\mathrm{M}+\mathrm{H})^{+}$calcd. for $\mathrm{C}_{28} \mathrm{H}_{41} \mathrm{~N}_{7} \mathrm{O}_{5} \mathrm{~S}_{5}$ : 716.1851, found: 716.1854; ${ }^{1} \mathrm{H}$ NMR (400 MHz, $\left.\mathrm{CD}_{3} \mathrm{OD}\right)$ $\delta$ o $0.75-0.86(\mathrm{~m}, 3 \mathrm{H}), 1.15-1.26(\mathrm{~m}, 4 \mathrm{H}), 1.42(\mathrm{~d}, J=6.8 \mathrm{~Hz}, 3 \mathrm{H}), 1.68-1.78$ (m, 1H), 1.89-2.15 (m, 3H), $1.94(\mathrm{~s}, 3 \mathrm{H}), 2.27(\mathrm{~s}, 3 \mathrm{H}), 3.00(\mathrm{~s}, 3 \mathrm{H}), 3.09$ $(\mathrm{dd}, J=8.2,6.2 \mathrm{~Hz}, 1 \mathrm{H}), 3.24-3.31(\mathrm{~m}, 1 \mathrm{H}), 3.50(\mathrm{dd}, J=10.2,3.2 \mathrm{~Hz}, 1 \mathrm{H})$, $3.72(\mathrm{~d}, J=14.3 \mathrm{~Hz}, 1 \mathrm{H}), 3.79$ (br dd, $J=3.2,1.0 \mathrm{~Hz}, 1 \mathrm{H}), 3.85(\mathrm{~d}, J=14.3 \mathrm{~Hz}$, $1 \mathrm{H}), 4.01(\mathrm{dd}, J=10.2,5.5 \mathrm{~Hz}, 1 \mathrm{H}), 4.13-4.21(\mathrm{~m}, 1 \mathrm{H}), 4.28-4.34(\mathrm{~m}, 1 \mathrm{H})$, $4.44(\mathrm{dd}, J=8.8,4.6 \mathrm{~Hz}, 1 \mathrm{H}), 5.16(\mathrm{~d}, J=5.5 \mathrm{~Hz}, 1 \mathrm{H}, 8.03(\mathrm{~s}, 1 \mathrm{H})$ and 8.69 $(\mathrm{s}, 1 \mathrm{H})$.

\section{$\mathbf{1}^{\prime}$-N-(tert-butoxycarbonyl)-1'-demethyl-4'-propyllincomycin (46)}

Compound $17(1.55 \mathrm{~g}, 5.19 \mathrm{mmol}), \quad$-hydroxybenzotriazole $(1.05 \mathrm{~g}$, $7.78 \mathrm{mmol}), N, N^{\prime}$-dicyclohexylcarbodiimide $(1.61 \mathrm{~g}, 7.78 \mathrm{mmol})$ and MTL $(1.97 \mathrm{~g}, 7.78 \mathrm{mmol})$ in DMF $(15.0 \mathrm{ml})$ were treated for $14 \mathrm{~h}$ according to the similar procedure as described for the preparation of $\mathbf{1 8}$ to afford $\mathbf{4 6}$. The total amount of this compound was used without purification to synthesize 47. For the qualified analytical purpose, the above crude 46 was purified by column chromatography (ethyl acetate only) to obtain the title compound as a colorless solid. ESI-MS $\mathrm{m} / z$ $535(\mathrm{M}+\mathrm{H})^{+}$as $\mathrm{C}_{25} \mathrm{H}_{46} \mathrm{~N}_{2} \mathrm{O}_{8} \mathrm{~S} ;{ }^{1} \mathrm{H}$ NMR $(400 \mathrm{MHz}$, $\left.\mathrm{CD}_{3} \mathrm{OD}\right) \delta 0.76-0.90(\mathrm{~m}, 6 \mathrm{H}), 1.04-1.32(\mathrm{~m}, 11 \mathrm{H}), 1.35,1.36(\mathrm{~s} \times 2,9 \mathrm{H})$, $1.57-1.72(\mathrm{~m}, 1 \mathrm{H}), 1.97,1.99(\mathrm{~s} \mathrm{x} \mathrm{2,3H}), 2.00-2.15(\mathrm{~m}, 1 \mathrm{H}), 3.03$ (br t, $J=10.4 \mathrm{~Hz}, 1 \mathrm{H}), 3.29-3.56(\mathrm{~m}, 2 \mathrm{H}), 3.67-3.90(\mathrm{~m}, 1 \mathrm{H}), 3.91-4.36(\mathrm{~m}, 5 \mathrm{H})$ and 5.15 (br d, $J=5.4 \mathrm{~Hz}, 1 \mathrm{H})$.

\section{$1^{\prime}$ - $N$-(tert-butoxycarbonyl)-1'-demethyl-4'-propyl-2,3,4-tris- $O$ - (trimethylsilyl)lincomycin (47)}

Compound 46 (crude), trimethylchlorosilane $(3.32 \mathrm{ml}, 25.9 \mathrm{mmol})$ and hexamethyldisilazane $(5.44 \mathrm{ml}, 25.9 \mathrm{mmol})$ in pyridine $(5.0 \mathrm{ml})$ were treated for $1 \mathrm{~h}$ according to the similar procedure as described for the preparation of $\mathbf{2 3}$ and then the crude compound and $6 \mathrm{~N}$ acetic acid $(1.55 \mathrm{ml})$ in methanol $(29.4 \mathrm{ml})$ were treated for $1 \mathrm{~h}$ according to the similar procedure as described for the preparation of $\mathbf{2 3}$ to afford $\mathbf{4 7}(1.66 \mathrm{~g}, 41.6 \%$ in three steps from 17) as a colorless solid. ESI-MS $m / z 751(\mathrm{M}+\mathrm{H})^{+}$as $\mathrm{C}_{34} \mathrm{H}_{70} \mathrm{~N}_{2} \mathrm{O}_{8} \mathrm{SSi}_{3} ;{ }^{1} \mathrm{H} \mathrm{NMR}$ $\left(400 \mathrm{MHz}, \mathrm{CDCl}_{3}\right) \delta 0.14(\mathrm{~s}, 18 \mathrm{H}), 0.18(\mathrm{~s}, 9 \mathrm{H}), 0.82-0.98(\mathrm{~m}, 6 \mathrm{H}), 1.08-1.35$ $(\mathrm{m}, 11 \mathrm{H}), 1.46(\mathrm{~s}, 9 \mathrm{H}), 1.80-2.35(\mathrm{~m}, 1 \mathrm{H}), 2.06(\mathrm{~s}, 3 \mathrm{H}), 2.66-3.30(\mathrm{~m}, 2 \mathrm{H})$, $3.36-4.41(\mathrm{~m}, 8 \mathrm{H})$ and $5.13-5.24(\mathrm{~m}, 1 \mathrm{H})$.

\section{7(S)-1'-demethyl-7-deoxy-4' -n-propyl-7-\{5-(2-nitrophenyl)-1,3,4-} thiadiazol-2-ylthio\}lincomycin (48)

To a solution of compound 47 ( $400 \mathrm{mg}, 0.53 \mathrm{mmol})$ in THF $(4 \mathrm{ml})$ at $0{ }^{\circ} \mathrm{C}$ were added triphenylphosphine $(209.6 \mathrm{mg}, 0.80 \mathrm{mmol})$, diethylazodicarboxylate $(0.15 \mathrm{ml}, 0.80 \mathrm{mmol}), 5$-(2-nitrophenyl)-1,3,4-thiadiazole-2-thiol $(296.7 \mathrm{mg}$, $1.24 \mathrm{mmol})$ and stirred at room temperature for $6 \mathrm{~h}$. The solution was purified by preparative TLC (hexane/ethyl acetate $=2 / 1$ ) to obtain $7(S)-1^{\prime}-N-($ tertbutoxycarbonyl)-1'-demethyl-7-deoxy-4'-n-propyl-7-\{5-(2-nitrophenyl)-1,3,4thiadiazol-2-ylthio\}-2,3,4-tris-O-(trimethylsilyl)lincomycin (235.4 mg with unseparable impurity). To this crude compound $(235.4 \mathrm{mg} 0.24 \mathrm{mmol}$ ) was added 2,2,2-trifluoroacetic acid $(1 \mathrm{ml})$ at $0{ }^{\circ} \mathrm{C}$ and stirred at room temperature for $30 \mathrm{~min}$. The solution was concentrated under reduced pressure. The resulting residue was purified by preparative TLC $\left(\mathrm{CHCl}_{3} / \mathrm{MeOH} / 28 \%\right.$ aq $\left.\mathrm{NH}_{4} \mathrm{OH}=9 / 2 / 0.2\right)$ to obtain the title compound $(57.3 \mathrm{mg}, 36.1 \%$ in 2 steps from 47) as a colorless solid. $[\alpha]_{\mathrm{D}}{ }^{26}+91.0^{\circ}(c 0.40, \mathrm{MeOH})$; ESI-MS $\mathrm{m} / z 656$ $(\mathrm{M}+\mathrm{H})^{+}$as $\mathrm{C}_{28} \mathrm{H}_{41} \mathrm{~N}_{5} \mathrm{O}_{7} \mathrm{~S}_{3}$; TOF-ESI-HRMS $(\mathrm{M}+\mathrm{H})^{+}$calcd. for $\mathrm{C}_{28} \mathrm{H}_{41} \mathrm{~N}_{5} \mathrm{O}_{7} \mathrm{~S}_{3}$ : 656.2246, found: 656.2239; ${ }^{1} \mathrm{H}$ NMR $\left(400 \mathrm{MHz}, \mathrm{CD}_{3} \mathrm{OD}\right)$ $\delta \quad 0.86-0.97(\mathrm{~m}, 6 \mathrm{H}), 1.22-1.42(\mathrm{~m}, 8 \mathrm{H}), 1.56(\mathrm{~d}, J=6.8 \mathrm{~Hz}, 3 \mathrm{H}), 1.63$ (dd, $J=13.0,8.2 \mathrm{~Hz}, 1 \mathrm{H}), 2.00(\mathrm{~s}, 3 \mathrm{H}), 2.11-2.19(\mathrm{~m}, 1 \mathrm{H}), 2.79-2.88(\mathrm{~m}, 2 \mathrm{H})$, $3.56(\mathrm{dd}, J=10.2,3.2 \mathrm{~Hz}, 1 \mathrm{H}), 3.80-3.86(\mathrm{~m}, 1 \mathrm{H}), 3.93(\mathrm{t}, J=8.4 \mathrm{~Hz}, 1 \mathrm{H}), 4.10$ (dd, $J=10.2,5.6 \mathrm{~Hz}, 1 \mathrm{H}), 4.38-4.43(\mathrm{~m}, 1 \mathrm{H}), 4.45(\mathrm{dq}, J=6.8,2.7 \mathrm{~Hz}, 1 \mathrm{H})$, $4.64(\mathrm{dd}, J=10.0,2.7 \mathrm{~Hz}, 1 \mathrm{H}), 5.27(\mathrm{~d}, J=5.6 \mathrm{~Hz}, 1 \mathrm{H}), 7.75-7.86(\mathrm{~m}, 3 \mathrm{H})$ and $8.07-8.13(\mathrm{~m}, 1 \mathrm{H})$.

\section{$1^{\prime}-N$-(tert-butoxycarbonyl)-4'-\{3-(tert-butyldimethylsilyloxy)} propyl\}-1'-demethyl-4'-depropyllincomycin (49)

Compound 11 (3.02, crude), 1-hydroxybenzotriazole (1.35 g, $10.01 \mathrm{mmol}), \mathrm{N}$, $N^{\prime}$-dicyclohexylcarbodiimide $(2.07 \mathrm{~g}, 10.01 \mathrm{mmol})$ and MTL $(2.54 \mathrm{~g}$, $10.01 \mathrm{mmol})$ in DMF $(15.0 \mathrm{ml})$ were treated for $13 \mathrm{~h}$ according to the similar procedure as described for the preparation of 19 to afford $49(3.0 \mathrm{~g}, 62.5 \%$ in 3 steps from 8) as a colorless solid. ESI-MS $m / z 623(\mathrm{M}+\mathrm{H})^{+}$as $\mathrm{C}_{28} \mathrm{H}_{54} \mathrm{~N}_{2} \mathrm{O}_{9} \mathrm{SSi}$; ${ }^{1} \mathrm{H}$ NMR $\left(400 \mathrm{MHz}, \mathrm{CD}_{3} \mathrm{OD}\right) \delta 0.06(\mathrm{~s}, 6 \mathrm{H}), 0.90(\mathrm{~s}, 9 \mathrm{H}), 1.15-1.27(\mathrm{~m}, 3 \mathrm{H})$, 1.37-1.64 (m, 4H), 1.44, 1.47 (s x 2, 9H), 1.74-1.91 (m, 1H), 2.06, 2.07 ( $\mathrm{s} \times 2$, $3 \mathrm{H}), 2.09-2.18(\mathrm{~m}, 1 \mathrm{H}), 2.30-2.45(\mathrm{~m}, 1 \mathrm{H}), 2.94(\mathrm{br} \mathrm{t}, J=10.0 \mathrm{~Hz}, 1 \mathrm{H})$, $3.53-3.72(\mathrm{~m}, 4 \mathrm{H}), 3.74-3.93(\mathrm{~m}, 1 \mathrm{H}), 3.98-4.17(\mathrm{~m}, 3 \mathrm{H}), 4.24$ (br dd, $J=8.9$, $1.3 \mathrm{~Hz}, 1 \mathrm{H}), 4.27-4.46(\mathrm{~m}, 1 \mathrm{H})$ and $5.24(\mathrm{~d}, J=5.4 \mathrm{~Hz}, 1 \mathrm{H})$.

$1^{\prime}-N$-(tert-butoxycarbonyl)-1'-demethyl-4'-depropyl-4' - $\{03-($ tertbutyldimethylsilyloxy)propyl\}- 2,3,4-tris-O-(trimethylsilyl) lincomycin (50)

Compound 49 ( $3.0 \mathrm{~g}, 4.82 \mathrm{mmol})$, trimethylchlorosilane $(3.08 \mathrm{ml}, 24.1 \mathrm{mmol})$ and hexamethyldisilazane $(5.05 \mathrm{ml}, 24.1 \mathrm{mmol})$ in pyridine $(10 \mathrm{ml})$ were treated for $20 \mathrm{~min}$ according to the similar procedure as described for the preparation of 23 and then the crude compound and $6 \mathrm{~N}$ acetic acid $(1.45 \mathrm{ml})$ in methanol $(27 \mathrm{ml})$ were treated for $80 \mathrm{~min}$ according to the similar procedure 
as described for the preparation of $\mathbf{2 3}$ to afford $\mathbf{5 0}(3.02 \mathrm{~g}, 74.8 \%$ in two steps from 49) as a colorless solid. ESI-MS $m / z 839(\mathrm{M}+\mathrm{H})^{+}$as $\mathrm{C}_{37} \mathrm{H}_{78} \mathrm{~N}_{2} \mathrm{O}_{9} \mathrm{SSi}_{4} ;{ }^{1} \mathrm{H}$ NMR $\left(400 \mathrm{MHz}, \mathrm{CDCl}_{3}\right) \delta 0.04(\mathrm{~s}, 6 \mathrm{H}), 0.07-0.20(\mathrm{~m}, 27 \mathrm{H}), 0.89(\mathrm{~s}, 9 \mathrm{H})$, 1.05-1.22 (m, 3H), 1.35-1.90 (m, 5H), 1.48 (s, 9H), 2.07 (s, 3H), 2.11-3.25 (m, 3H), 3.44-3.66 (m, 4H), 3.70-3.89 (m, 1H), 3.91-4.18 (m, 3H), 4.19-4.42 $(\mathrm{m}, 2 \mathrm{H})$ and $5.19(\mathrm{br} \mathrm{d}, J=5.6 \mathrm{~Hz}, 1 \mathrm{H})$.

\section{$7(S)-1^{\prime}-N$-(tert-butoxycarbonyl)-1'-demethyl-7-deoxy-4'-depropyl- 4'-(3-hydroxypropyl)-7-\{5-(2-nitrophenyl)-1,3,4-thiadiazol-2-ylthio $\}$ lincomycin (51)}

To a solution of compound $\mathbf{5 0}(2.87 \mathrm{~g}, 3.41 \mathrm{mmol})$ in THF $(15 \mathrm{ml})$ at $0{ }^{\circ} \mathrm{C}$ were added triphenylphosphine $(1.34 \mathrm{~g}, 5.12 \mathrm{mmol})$, diethylazodicarboxylate $(932 \mu \mathrm{l}, 5.12 \mathrm{mmol})$ and 5-(2-nitrophenyl)-1,3,4-thiadiazole-2-thiol (1.26 g, $5.29 \mathrm{mmol}$ ) and stirred at room temperature for $10 \mathrm{~h}$. The solution was substituted by toluene, purified by silica gel column chromatography (hexane to hexane/ethyl acetate $=4 / 1$ ) to obtain $1^{\prime}-N$ - (tert-butoxycarbonyl)4'-\{3-(tert-butyldimethylsilyloxy)propyl\}-1'-demethyl-7-deoxy-4'-depropyl-7\{5-(2-nitrophenyl)-1,3,4-thiadiazol-2-ylthio\}-2,3,4-tris- $O$-(trimethylsilyl)lincomycin $(2.62 \mathrm{~g}$, crude). To this crude compound $(2.62 \mathrm{~g})$ was added $1 \mathrm{~m}$ THF solution of tetra- $n$-butyl ammonium fluoride $(14.8 \mathrm{ml}, 14.8 \mathrm{mmol})$ and acetic acid $(0.848 \mathrm{ml}, 14.8 \mathrm{mmol})$ and stirred at room temperature for $5 \mathrm{~h}$. The mixture was diluted with brine and ethyl acetate, extracted with ethyl acetate, and then the organic phase was dried over $\mathrm{Na}_{2} \mathrm{SO}_{4}$, filtrated and concentrated under reduced pressure. The resulting residue was purified by silica gel column chromatography (hexane/ethyl acetate $=1 / 1$ to ethyl acetate only to ethyl acetate $/ \mathrm{MeOH}=10 / 1)$ to obtain the title compound (1.74 g with unseparable impurity $\left(96 \%\right.$ in two steps as reference yield)). FAB-MS $m / z 752(\mathrm{M}+\mathrm{Na})^{+}$as $\mathrm{C}_{30} \mathrm{H}_{43} \mathrm{~N}_{5} \mathrm{O}_{10} \mathrm{~S}_{3}$.

$7(S)-4^{\prime}$-(3-aminopropyl)-1'-N-(tert-butoxycarbonyl)-1'-demethyl-7deoxy-4'-depropyl-7-\{5-(2-nitrophenyl)-1,3,4-thiadiazol-2-ylthio $\}$ lincomycin (52)

To a solution of compound 51 (500 mg, $0.685 \mathrm{mmol}$ ), sodium azide (223 mg, $3.43 \mathrm{mmol})$ and triphenylphosphine $(359.3 \mathrm{mg}, 1.37 \mathrm{mmol})$ in DMF $(7 \mathrm{ml})$ was added tetrabromomethane $(454.3 \mathrm{mg}, 1.37 \mathrm{mmol})$ and stirred at $50^{\circ} \mathrm{C}$ for $2 \mathrm{~h}$. The mixture was diluted with brine and ethyl acetate, extracted with ethyl acetate and then the organic phase was dried over $\mathrm{Na}_{2} \mathrm{SO}_{4}$, filtrated and concentrated under reduced pressure. The resulting residue was purified by silica gel column chromatography (chloroform $/ \mathrm{MeOH}=10 / 1$ ) to obtain 7(S)-4'-(3-azidopropyl)-1'-N-(tert-butoxycarbonyl)-1'-demethyl-7-deoxy-4'depropyl-7-\{5-(2-nitrophenyl)-1,3,4-thiadiazol-2-ylthio\}lincomycin (468 mg with unseparable impurity ( $91 \%$ as reference yield)). To a solution of this crude compound $(239 \mathrm{mg}, 0.317 \mathrm{mmol})$ in THF $(3 \mathrm{ml})$ was added triphenylphosphine $(249.0 \mathrm{mg}, 0.95 \mathrm{mmol})$, stirred at room temperature for $1 \mathrm{~h}$, and then $\mathrm{H}_{2} \mathrm{O}$ was added and stirred at $50^{\circ} \mathrm{C}$ for $2 \mathrm{~h}$. The mixture was diluted with brine, ethyl acetate and $1 \mathrm{~N} \mathrm{HCl}(400 \mu \mathrm{l})$, washed with ethyl acetate and then the aqueous phase was added to the saturated aqueous $\mathrm{NaHCO}_{3}$, extracted with $\mathrm{CHCl}_{3}$, dried over $\mathrm{Na}_{2} \mathrm{SO}_{4}$, filtrated and concentrated under reduced pressure to obtain the title compound (229 mg with unseparable impurity ( $99 \%$ as reference yield)).

7(S)-1'-demethyl-7-deoxy-4'-depropyl-4' -(3-dimethylaminopropyl)7-\{5-(2-nitrophenyl)-1,3,4-thiadiazol-2-ylthio\}lincomycin (53)

Compound $52(69.0 \mathrm{mg}, 0.093 \mathrm{mmol}), 36 \%$ aqueous formaldehyde $(23.1 \mu \mathrm{l}$, $0.28 \mathrm{mmol}), \mathrm{AcOH}(79.5 \mu \mathrm{l}, 1.39 \mathrm{mmol})$ and $\mathrm{NaBH}(\mathrm{OAc})_{3}(294.3 \mathrm{mg}$, $1.39 \mathrm{mmol})$ in $\mathrm{MeOH}(1 \mathrm{ml})$ were treated at room temperature for $20 \mathrm{~min}$ according to the similar procedure as described for the preparation of $\mathbf{3 8}$ to afford $7(S)-1^{\prime}-N$-(tert-butoxycarbonyl)-1'-demethyl-7-deoxy-4'-depropyl-4'-(3dimethylaminopropyl)-7-\{5-(2-nitrophenyl)-1,3,4-thiadiazol-2-ylthio\}lincomycin $(75.0 \mathrm{mg}$, crude). To this crude compound $(75.0 \mathrm{mg}$ ) was added $2,2,2$ trifluoroacetic acid $(1 \mathrm{ml})$ at $0{ }^{\circ} \mathrm{C}$ and stirred at room temperature for $30 \mathrm{~min}$. The solution was concentrated under reduced pressure. The resulting residue was purified by preparative TLC $\left(\mathrm{CHCl}_{3} / \mathrm{MeOH} / 28 \%\right.$ aq. $\left.\mathrm{NH}_{4} \mathrm{OH}=9 / 4 / 0.4\right)$ to obtain the title compound $(35.0 \mathrm{mg}, 57.6 \%$ in two steps from 52$)$ as a colorless

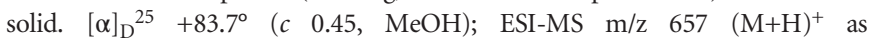

$\mathrm{C}_{27} \mathrm{H}_{40} \mathrm{~N}_{6} \mathrm{O}_{7} \mathrm{~S}_{3}$; TOF-ESI-HRMS $(\mathrm{M}+\mathrm{H})^{+}$calcd. for $\mathrm{C}_{27} \mathrm{H}_{40} \mathrm{~N}_{6} \mathrm{O}_{7} \mathrm{~S}_{3}$ : 657.2199, found: $657.2193 ;{ }^{1} \mathrm{H}$ NMR $\left(400 \mathrm{MHz}, \mathrm{CD}_{3} \mathrm{OD}\right) \delta 1.34-1.44(\mathrm{~m}, 2 \mathrm{H}), 1.48-1.64$ $(\mathrm{m}, 2 \mathrm{H}), 1.57(\mathrm{~d}, J=7.0 \mathrm{~Hz}, 3 \mathrm{H}), 1.76-1.88(\mathrm{~m}, 1 \mathrm{H}), 2.01(\mathrm{~s}, 3 \mathrm{H}), 2.04-2.13$ $(\mathrm{m}, 2 \mathrm{H}), 2.34(\mathrm{~s}, 6 \mathrm{H}), 2.46(\mathrm{t}, J=7.8 \mathrm{~Hz}, 2 \mathrm{H}), 2.56(\mathrm{dd}, J=10.3,7.8 \mathrm{~Hz}, 1 \mathrm{H})$, $3.24(\mathrm{dd}, J=10.3,6.8 \mathrm{~Hz}, 1 \mathrm{H}), 3.56(\mathrm{dd}, J=10.2,3.3 \mathrm{~Hz}, 1 \mathrm{H}), 3.81(\mathrm{dd}, J=9.3$, $3.9 \mathrm{~Hz}, 1 \mathrm{H}), 3.83$ (br dd, $J=3.3,0.8 \mathrm{~Hz}, 1 \mathrm{H}), 4.10$ (dd, $J=10.2,5.6 \mathrm{~Hz}, 1 \mathrm{H})$, 4.41 (br dd, $J=9.9,0.8 \mathrm{~Hz}, 1 \mathrm{H}), 4.46$ (dq, $J=7.0,2.8 \mathrm{~Hz}, 1 \mathrm{H}), 4.62$ (dd, $J=9.9$, $2.8 \mathrm{~Hz}, 1 \mathrm{H}), 5.28(\mathrm{~d}, J=5.6 \mathrm{~Hz}, 1 \mathrm{H}), 7.75-7.87(\mathrm{~m}, 3 \mathrm{H})$ and $8.06-8.15$ $(\mathrm{m}, 1 \mathrm{H})$.

7(S)-1'-demethyl-7-deoxy-4'-depropyl-4'-(3-methoxypropyl)-7-\{5(2-nitrophenyl)-1,3,4-thiadiazol-2-ylthio\}lincomycin (54)

Compound 24 (266.0 mg, $0.36 \mathrm{mmol}$ ), triphenylphosphine $(142.0 \mathrm{mg}$, $0.54 \mathrm{mmol})$, diethylazodicarboxylate $(98 \mu \mathrm{l}, 0.54 \mathrm{mmol})$ and 5-(2-nitrophenyl)-1,3,4-thiadiazole-2-thiol $(132.0 \mathrm{mg}, 0.56 \mathrm{mmol})$ in THF $(2 \mathrm{ml})$ were treated for $18 \mathrm{~h}$ according to the similar procedure as described for the preparation of 31 to afford $7(S)-1^{\prime}-N$-(tert-butoxycarbonyl)-1'-demethyl-7deoxy-4'-depropyl-4'-(3-methoxypropyl)-7-\{5-(2-nitrophenyl)-1,3,4-thiadiazol-2-ylthio\}-2,3,4-tris- $O$-(trimethylsilyl)lincomycin ( $325.0 \mathrm{mg}$ as crude). To this crude compound $(325.0 \mathrm{mg})$ was added 2,2,2-trifluoroacetic acid $(1 \mathrm{ml})$ at $0{ }^{\circ} \mathrm{C}$ and stirred at room temperature for $15 \mathrm{~min}$. The solution was concentrated under reduced pressure. The resulting residue was purified by preparative TLC $\left(\mathrm{CHCl}_{3} / \mathrm{MeOH} / 28 \%\right.$ aq. $\left.\mathrm{NH}_{4} \mathrm{OH}=9 / 2 / 0.2\right)$ to obtain the title compound (112.0 mg, $48.3 \%$ in two steps from 24$)$ as a colorless solid. $[\alpha]_{D}{ }^{24}+82.6^{\circ}$ (c 0.45, MeOH); ESI-MS m/z $644(\mathrm{M}+\mathrm{H})^{+}$as $\mathrm{C}_{26} \mathrm{H}_{37} \mathrm{~N}_{5} \mathrm{O}_{8} \mathrm{~S}_{3}$; TOF-ESI-HRMS $(\mathrm{M}+\mathrm{H})^{+}$calcd. for $\mathrm{C}_{26} \mathrm{H}_{37} \mathrm{~N}_{5} \mathrm{O}_{8} \mathrm{~S}_{3}:$ 644.1883, found: 644.1880; ${ }^{1} \mathrm{H}$ NMR $\left(400 \mathrm{MHz}, \mathrm{CD}_{3} \mathrm{OD}\right) \quad \delta \quad 1.40-1.52(\mathrm{~m}, \quad 2 \mathrm{H}), \quad 1.54-1.65 \quad(\mathrm{~m}, \quad 2 \mathrm{H}), \quad 1.57$ (d, $J=6.9 \mathrm{~Hz}, 3 \mathrm{H}), 1.89-1.97(\mathrm{~m}, 1 \mathrm{H}), 2.01(\mathrm{~s}, 3 \mathrm{H}), 2.09-2.23(\mathrm{~m}, 2 \mathrm{H}), 2.68$ (dd, $J=10.7,8.0 \mathrm{~Hz}, 1 \mathrm{H}), 3.30(\mathrm{~s}, 3 \mathrm{H}), 3.32-3.36(\mathrm{~m}, 1 \mathrm{H}), 3.39(\mathrm{t}, J=6.2,2 \mathrm{H})$, 3.57 (dd, $J=10.2,3.2 \mathrm{~Hz}, 1 \mathrm{H}), 3.85$ (br dd, $J=3.2,0.8 \mathrm{~Hz}, 1 \mathrm{H}), 4.02$ (dd, $J=9.1,4.1 \mathrm{~Hz}, 1 \mathrm{H}), 4.11(\mathrm{dd}, J=10.2,5.6 \mathrm{~Hz}, 1 \mathrm{H}), 4.42(\mathrm{br} \mathrm{dd}, J=10.0$, $0.8 \mathrm{~Hz}, 1 \mathrm{H}), 4.47(\mathrm{dq}, J=6.9,2.7 \mathrm{~Hz}, 1 \mathrm{H}), 4.66(\mathrm{dd}, J=10.0,2.7 \mathrm{~Hz}, 1 \mathrm{H}), 5.28$ $(\mathrm{d}, J=5.6 \mathrm{~Hz}, 1 \mathrm{H}), 7.76-7.87(\mathrm{~m}, 3 \mathrm{H})$ and $8.06-8.14(\mathrm{~m}, 1 \mathrm{H})$.

\section{7(S)-4'-i-butyl-1'-demethyl-7-deoxy-4'-depropyl-7-(5-(2-} nitrophenyl)-1,3,4-thiadiazol-2-yl)thiolincomycin (55)

Compound 25 (204.0 mg, $0.28 \mathrm{mmol})$, triphenylphosphine $(109.1 \mathrm{mg}$, $0.42 \mathrm{mmol})$, diethylazodicarboxylate $(75.8 \mu \mathrm{l}, 0.42 \mathrm{mmol})$ and 5-(2-nitrophenyl)-1,3,4-thiadiazole-2-thiol $(103.0 \mathrm{mg}, 0.43 \mathrm{mmol})$ in THF $(2 \mathrm{ml})$ were treated for $14.5 \mathrm{~h}$ according to the similar procedure as described for the preparation of 31 to afford 7(S)-1'-N-(tert-butoxycarbonyl)-4'-i-butyl-1'demethyl-7-deoxy-4'-depropyl-7-\{5-(2-nitrophenyl)-1,3,4-thiadiazol-2-ylthio\}2,3,4-tris- $\mathrm{O}$-(trimethylsilyl)lincomycin ( $170.0 \mathrm{mg}$ as crude). To this crude compound $(170.0 \mathrm{mg})$ was added 2,2,2-trifluoroacetic acid $(1 \mathrm{ml})$ at $0{ }^{\circ} \mathrm{C}$ and stirred at room temperature for $30 \mathrm{~min}$. The solution was concentrated under reduced pressure. The resulting residue was purified by preparative TLC $\left(\mathrm{CHCl}_{3} / \mathrm{MeOH} / 28 \%\right.$ aq. $\left.\mathrm{NH}_{4} \mathrm{OH}=9 / 2 / 0.2\right)$ to obtain the title compound $(39.0 \mathrm{mg}, 22.5 \%$ in two steps from 25$)$ as a colorless solid. $[\alpha]_{D}{ }^{24}+85.8^{\circ}$ (c 0.54, MeOH); ESI-MS m/z $628(\mathrm{M}+\mathrm{H})^{+}$as $\mathrm{C}_{26} \mathrm{H}_{37} \mathrm{~N}_{5} \mathrm{O}_{7} \mathrm{~S}_{3}$; TOF-ESI-HRMS $(\mathrm{M}+\mathrm{H})^{+}$calcd. for $\mathrm{C}_{26} \mathrm{H}_{37} \mathrm{~N}_{5} \mathrm{O}_{7} \mathrm{~S}_{3}: 628.1933$, found: 628.1936; ${ }^{1} \mathrm{H}$ NMR $\left(400 \mathrm{MHz}, \mathrm{CD}_{3} \mathrm{OD}\right) \delta 0.90(\mathrm{~d}, J=5.3 \mathrm{~Hz}, 3 \mathrm{H}), 0.91(\mathrm{~d}, J=5.4 \mathrm{~Hz}, 3 \mathrm{H})$, $1.23-1.34(\mathrm{~m}, 2 \mathrm{H}), 1.53-1.64(\mathrm{~m}, 1 \mathrm{H}), 1.57(\mathrm{~d}, J=6.8 \mathrm{~Hz}, 3 \mathrm{H}), 1.81-1.92$ $(\mathrm{m}, 1 \mathrm{H}), 2.00(\mathrm{~s}, 3 \mathrm{H}), 2.04-2.15(\mathrm{~m}, 1 \mathrm{H}), 2.15-2.27(\mathrm{~m}, 1 \mathrm{H}), 2.58(\mathrm{dd}, J=10.4$, $8.7 \mathrm{~Hz}, 1 \mathrm{H}), 3.26-3.30(\mathrm{~m}, 1 \mathrm{H}), 3.56(\mathrm{dd}, J=10.2,3.2 \mathrm{~Hz}, 1 \mathrm{H}), 3.84(\mathrm{br} \mathrm{dd}$, $J=3.2,0.8 \mathrm{~Hz}, 1 \mathrm{H}), 3.92(\mathrm{dd}, J=9.2,4.3 \mathrm{~Hz}, 1 \mathrm{H}), 4.10(\mathrm{dd}, J=10.2,5.6 \mathrm{~Hz}$, $1 \mathrm{H}), 4.41$ (br dd, $J=10.0,0.8 \mathrm{~Hz}, 1 \mathrm{H}), 4.47(\mathrm{dq}, J=6.8,2.8 \mathrm{~Hz}, 1 \mathrm{H}), 4.65$ $(\mathrm{dd}, J=10.0,2.8 \mathrm{~Hz}, 1 \mathrm{H}), 5.28(\mathrm{~d}, J=5.6 \mathrm{~Hz}, 1 \mathrm{H}), 7.76-7.87(\mathrm{~m}, 3 \mathrm{H})$ and $8.08-8.13(\mathrm{~m}, 1 \mathrm{H})$.

\section{7(S)-1'-demethyl-7-deoxy-4' -depropyl-7-\{5-(2-nitrophenyl)-1,3,4-} thiadiazol-2-ylthio $\}-4^{\prime}$ - $n$-pentyllincomycin (56)

Compound $26 \quad(400 \mathrm{mg}, \quad 0.54 \mathrm{mmol})$, triphenylphosphine $(213.5 \mathrm{mg}$, $0.81 \mathrm{mmol})$, diethylazodicarboxylate $(150 \mu \mathrm{l}, 0.81 \mathrm{mmol})$ and 5-(2-nitrophenyl)-1,3,4-thiadiazole-2-thiol $(201.4 \mathrm{mg}, 0.84 \mathrm{mmol})$ in THF $(4 \mathrm{ml})$ were treated for $24 \mathrm{~h}$ according to the similar procedure as described for the preparation of 31 to afford 7(S)-1'-N-(tert-butoxycarbonyl)-1'-demethyl-7- 
deoxy-4'-depropyl-7-\{5-(2-nitrophenyl)-1,3,4-thiadiazol-2-ylthio $\}$-4' -n-pentyl2,3,4-tris- $\mathrm{O}$-(trimethylsilyl)lincomycin ( $374.0 \mathrm{mg}$ as crude). To this crude compound $(374 \mathrm{mg})$ was added 2,2,2-trifluoroacetic acid $(1 \mathrm{ml})$ at $0{ }^{\circ} \mathrm{C}$ and stirred at room temperature for $30 \mathrm{~min}$. The solution was concentrated under reduced pressure. The resulting residue was purified by preparative TLC $\left(\mathrm{CHCl}_{3} / \mathrm{MeOH} / 28 \%\right.$ aq. $\left.\mathrm{NH}_{4} \mathrm{OH}=9 / 2 / 0.2\right)$ to obtain the title compound (72.6 mg, 20.9\% in two steps from 26) as a colorless solid. $[\alpha]_{\mathrm{D}}{ }^{25}+34.3^{\circ}$ (c $0.10, \mathrm{MeOH})$; ESI-MS m/z $642(\mathrm{M}+\mathrm{H})^{+}$as $\mathrm{C}_{27} \mathrm{H}_{39} \mathrm{~N}_{5} \mathrm{O}_{7} \mathrm{~S}_{3}$; TOF-ESI-HRMS $(\mathrm{M}+\mathrm{H})^{+}$calcd. for $\mathrm{C}_{27} \mathrm{H}_{39} \mathrm{~N}_{5} \mathrm{O}_{7} \mathrm{~S}_{3}$ : 642.2090, found: 642.2083; ${ }^{1} \mathrm{H}$ NMR $\left(400 \mathrm{MHz}, \quad \mathrm{CD}_{3} \mathrm{OD}\right) \quad \delta \quad 0.80-0.95 \quad(\mathrm{~m}, \quad 3 \mathrm{H}), \quad 1.21-1.43 \quad(\mathrm{~m}, \quad 8 \mathrm{H}), \quad 1.57$ $(\mathrm{d}, J=7.0 \mathrm{~Hz}, 3 \mathrm{H}), 1.81-1.95(\mathrm{~m}, 1 \mathrm{H}), 2.02-2.14(\mathrm{~m}, 2 \mathrm{H}), 2.09(\mathrm{~s}, 3 \mathrm{H}), 2.62$ (dd, $J=10.4,7.9 \mathrm{~Hz}, 1 \mathrm{H}), 3.27$ (dd, $J=10.5,6.8 \mathrm{~Hz}, 1 \mathrm{H}), 3.61$ (dd, $J=10.2$, $3.2 \mathrm{~Hz}, 1 \mathrm{H}$ ), 3.83 (br dd, $J=3.2,0.9 \mathrm{~Hz}, 1 \mathrm{H}), 3.91$ (dd, $J=9.1,4.2 \mathrm{~Hz}, 1 \mathrm{H})$, $4.14(\mathrm{dd}, J=10.2,5.6 \mathrm{~Hz}, 1 \mathrm{H}), 4.34-4.40(\mathrm{~m}, 1 \mathrm{H}), 4.42(\mathrm{dq}, J=7.0,2.9 \mathrm{~Hz}$, $1 \mathrm{H}), 4.57(\mathrm{dd}, J=10.0,2.9 \mathrm{~Hz}, 1 \mathrm{H}), 5.31(\mathrm{~d}, J=5.6 \mathrm{~Hz}, 1 \mathrm{H}), 7.71-7.87$ $(\mathrm{m}, 3 \mathrm{H})$ and $8.07-8.14(\mathrm{~m}, 1 \mathrm{H})$.

7(S)-7-deoxy-4'-depropyl-7-\{5-(2-nitrophenyl)-1,3,4-thiadiazol-2ylthio\}-4'-n-pentyllincomycin (57)

Compound $56(56.1 \mathrm{mg}, 0.087 \mathrm{mmol}), 36 \%$ aqueous formaldehyde $(22 \mu \mathrm{l}$, $0.26 \mathrm{mmol}), \mathrm{AcOH}(30 \mu \mathrm{l}, 0.52 \mathrm{mmol})$ and $\mathrm{NaBH}(\mathrm{OAc})_{3} \quad(55.5 \mathrm{mg}$, $0.26 \mathrm{mmol})$ in $\mathrm{MeOH}(0.6 \mathrm{ml})$ were treated at room temperature for $1 \mathrm{~h}$ according to the similar procedure as described for the preparation of $\mathbf{3 8}$ to afford $57(49.1 \mathrm{mg}, 85.7 \%)$ as a colorless solid. $[\alpha]_{\mathrm{D}}{ }^{25}+91.6^{\circ}(c 1.16, \mathrm{MeOH})$; ESI-MS $m / z 656(\mathrm{M}+\mathrm{H})^{+}$as $\mathrm{C}_{28} \mathrm{H}_{41} \mathrm{~N}_{5} \mathrm{O}_{7} \mathrm{~S}_{3}$; TOF-ESI-HRMS $(\mathrm{M}+\mathrm{H})^{+}$calcd. for $\mathrm{C}_{28} \mathrm{H}_{41} \mathrm{~N}_{5} \mathrm{O}_{7} \mathrm{~S}_{3}$ : 656.2246, found: $656.2238 ;{ }^{1} \mathrm{H}$ NMR (400 MHz, $\mathrm{CD}_{3} \mathrm{OD}$ ) $\delta$ 0.83-0.92 (m, 3H), $1.21-1.42(\mathrm{~m}, 8 \mathrm{H}), 1.57(\mathrm{~d}, J=7.0 \mathrm{~Hz}, 3 \mathrm{H}), 1.78-1.91$ (m, 1H), 1.96-2.10 (m, 2H), $2.01(\mathrm{~s}, 3 \mathrm{H}), 2.12-2.27(\mathrm{~m}, 1 \mathrm{H}), 2.39(\mathrm{~s}, 3 \mathrm{H}), 3.02$ (dd, $J=10.5,5.1 \mathrm{~Hz}, 1 \mathrm{H}), 3.23-3.29(\mathrm{~m}, 1 \mathrm{H}), 3.57(\mathrm{dd}, J=10.2,3.2 \mathrm{~Hz}, 1 \mathrm{H})$, $3.81(\mathrm{dd}, J=3.2,0.7 \mathrm{~Hz}, 1 \mathrm{H}), 4.11(\mathrm{dd}, J=10.2,5.6 \mathrm{~Hz}, 1 \mathrm{H}), 4.39-4.49$ $(\mathrm{m}, 1 \mathrm{H}), 4.60(\mathrm{dd}, J=9.8,3.2 \mathrm{~Hz}, 1 \mathrm{H}), 5.27(\mathrm{~d}, J=5.6,1 \mathrm{H}), 7.75-7.85(\mathrm{~m}, 3 \mathrm{H})$ and $8.06-8.11(\mathrm{~m}, 1 \mathrm{H})$.

\section{7(S)-1'-demethyl-7-deoxy-4'-depropyl-7-[5-\{5-(methylamino)} thiazol-4-yl\}-1,3,4-thiadiazol-2-ylthio]-4' -n-pentyllincomycin (58)

Compound 26 (400 mg, $0.54 \mathrm{mmol})$, triphenylphosphine $(213.5 \mathrm{mg}$, $0.81 \mathrm{mmol})$, diethylazodicarboxylate $(150 \mu \mathrm{l}, 0.81 \mathrm{mmol})$ and 5-\{5-(methylamino)thiazol-4-yl\}-1,3,4-thiadiazole-2-thiol $(193.9 \mathrm{mg}, 0.84 \mathrm{mmol})$ in THF $(4 \mathrm{ml})$ were treated for $24 \mathrm{~h}$ according to the similar procedure as described for the preparation of 31 to afford $7(S)-1^{\prime}-N$-(tert-butoxycarbonyl)- $1^{\prime}$ demethyl-7-deoxy-4'-depropyl-7-[5-\{5-(methylamino)thiazol-4-yl\}-1,3,4-thiadiazol-2-ylthio]-4'- $n$-pentyl-2,3,4-tris- $O$-(trimethylsilyl)lincomycin $\quad(109.8 \mathrm{mg}$, $21.3 \%)$. To the compound $(109.8 \mathrm{mg}, 0.12 \mathrm{mmol}$ ) was added 2,2,2-trifluoroacetic acid $(1 \mathrm{ml})$ at $0{ }^{\circ} \mathrm{C}$ and stirred at room temperature for $30 \mathrm{~min}$. The solution was concentrated under reduced pressure. The resulting residue was purified by preparative TLC $\left(\mathrm{CHCl}_{3} / \mathrm{MeOH} / 28 \%\right.$ aq. $\left.\mathrm{NH}_{4} \mathrm{OH}=9 / 2 / 0.2\right)$ to obtain the title compound $(59.0 \mathrm{mg}, 80.6 \%)$ as a colorless solid. $[\alpha]_{\mathrm{D}}^{25}+48.5^{\circ}$ (c $0.22, \mathrm{MeOH}$ ); ESI-MS m/z $633(\mathrm{M}+\mathrm{H})^{+}$as $\mathrm{C}_{25} \mathrm{H}_{40} \mathrm{~N}_{6} \mathrm{O}_{5} \mathrm{~S}_{4}$; TOF-ESI-HRMS $(\mathrm{M}+\mathrm{H})^{+}$calcd. for $\mathrm{C}_{25} \mathrm{H}_{40} \mathrm{~N}_{6} \mathrm{O}_{5} \mathrm{~S}_{4}$ : 633.2021, found: 633.2021; ${ }^{1} \mathrm{H}$ NMR $\left(400 \mathrm{MHz}, \quad \mathrm{CD}_{3} \mathrm{OD}\right) \quad \delta \quad 0.83-0.94(\mathrm{~m}, \quad 3 \mathrm{H}), \quad 1.21-1.43(\mathrm{~m}, \quad 8 \mathrm{H}), \quad 1.50$ $(\mathrm{d}, J=6.8 \mathrm{~Hz}, 3 \mathrm{H}), 1.80-1.91(\mathrm{~m}, 1 \mathrm{H}), 2.02(\mathrm{~s}, 3 \mathrm{H}), 2.02-2.13(\mathrm{~m}, 2 \mathrm{H}), 2.59$ (dd, $J=10.5,8.0 \mathrm{~Hz}, 1 \mathrm{H}), 3.12(\mathrm{~s}, 3 \mathrm{H}), 3.24-3.30(\mathrm{~m}, 1 \mathrm{H}), 3.58(\mathrm{dd}, J=10.2$, $3.2 \mathrm{~Hz}, 1 \mathrm{H}), 3.85$ (br dd, $J=3.2,0.7 \mathrm{~Hz}, 1 \mathrm{H}), 3.93(\mathrm{dd}, J=9.1,4.0 \mathrm{~Hz}, 1 \mathrm{H})$, $4.11(\mathrm{dd}, J=10.2,5.6 \mathrm{~Hz}, 1 \mathrm{H}), 4.29(\mathrm{dq}, J=6.8,2.7 \mathrm{~Hz}, 1 \mathrm{H}), 4.37-4.44$ $(\mathrm{m}, 1 \mathrm{H}), 4.61(\mathrm{dd}, J=10.0,2.7 \mathrm{~Hz}, 1 \mathrm{H}), 5.28(\mathrm{~d}, J=5.6 \mathrm{~Hz}, 1 \mathrm{H})$ and 8.13 (s, 1H).

\section{7(S)-7-deoxy-4'-depropyl-7-[5-\{5-(methylamino)thiazol-4-yl\}-1,3,4-} thiadiazol-2-ylthio]-4'-n-pentyllincomycin (59)

Compound 58 (35.8 mg, $0.057 \mathrm{mmol}), 36 \%$ aqueous formaldehyde $(14 \mu \mathrm{l}$, $0.17 \mathrm{mmol}), \mathrm{AcOH}(20 \mu \mathrm{l}, 0.34 \mathrm{mmol})$ and $\mathrm{NaBH}(\mathrm{OAc})_{3} \quad(36.0 \mathrm{mg}$, $0.17 \mathrm{mmol})$ in $\mathrm{MeOH}(0.5 \mathrm{ml})$ were treated at room temperature for $1 \mathrm{~h}$ according to the similar procedure as described for the preparation of $\mathbf{3 8}$ to afford $59(29.4 \mathrm{mg}, 80.3 \%)$ as an off-white solid. $[\alpha]_{D^{2}}^{25}+64.5^{\circ}(c 0.30, \mathrm{MeOH})$; ESI-MS $m / z 647(\mathrm{M}+\mathrm{H})^{+}$as $\mathrm{C}_{26} \mathrm{H}_{42} \mathrm{~N}_{6} \mathrm{O}_{5} \mathrm{~S}_{4}$; TOF-ESI-HRMS $(\mathrm{M}+\mathrm{H})^{+}$calcd. for $\mathrm{C}_{26} \mathrm{H}_{42} \mathrm{~N}_{6} \mathrm{O}_{5} \mathrm{~S}_{4}$ : 647.2178, found: 647.2176; ${ }^{1} \mathrm{H}$ NMR (400 MHz, $\mathrm{CD}_{3} \mathrm{OD}$ )

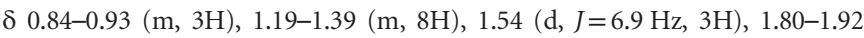
(m, 1H), $2.02(\mathrm{~s}, 3 \mathrm{H}), 1.96-2.21(\mathrm{~m}, 3 \mathrm{H}), 2.40(\mathrm{~s}, 3 \mathrm{H}), 3.06-3.14(\mathrm{~m}, 1 \mathrm{H}), 3.12$ $(\mathrm{s}, 3 \mathrm{H}), 3.21(\mathrm{dd}, J=7.9,5.5 \mathrm{~Hz}, 1 \mathrm{H}), 3.61(\mathrm{dd}, J=10.2,3.1 \mathrm{~Hz}, 1 \mathrm{H}), 3.84$ (dd, $J=3.1,0.6 \mathrm{~Hz}, 1 \mathrm{H}), 4.12$ (dd, $J=10.2,5.6 \mathrm{~Hz}, 1 \mathrm{H}), 4.28$ (dq, $J=6.9$, $2.9 \mathrm{~Hz}, 1 \mathrm{H}), \quad 4.43-4.44(\mathrm{~m}, 1 \mathrm{H}), 4.59(\mathrm{dd}, J=10.0,2.9 \mathrm{~Hz}, 1 \mathrm{H}), 5.28$ $(\mathrm{d}, J=5.6 \mathrm{~Hz}, 1 \mathrm{H})$ and $8.14(\mathrm{~s}, 1 \mathrm{H})$.

\section{$7(S)-1^{\prime}-N$-(tert-butoxycarbonyl)-1'-demethyl-7-deoxy-7-\{4- (methoxycarbonyl)phenylthio\}lincomycin (60)}

To a solution of compound $23(5.0 \mathrm{~g}, 7.05 \mathrm{mmol})$ in $\mathrm{CHCl}_{3}(22 \mathrm{ml})$ were added $\mathrm{Et}_{3} \mathrm{~N}(2.45 \mathrm{ml}, 17.6 \mathrm{mmol})$, methanesulfonyl chloride $(1.1 \mathrm{ml}$, $14.1 \mathrm{mmol}$ ) and stirred at room temperature for $30 \mathrm{~min}$. The mixture was added to saturated aqueous $\mathrm{NaHCO}_{3}$, extracted with $\mathrm{CHCl}_{3}$, dried over $\mathrm{Na}_{2} \mathrm{SO}_{4}$ and concentrated under reduced pressure. To a solution of crude compound in DMF $(50 \mathrm{ml})$ were added $\mathrm{K}_{2} \mathrm{CO}_{3}(2.92 \mathrm{~g}, 21.2 \mathrm{mmol})$ and methyl 4-mercaptobenzoate $(2.37 \mathrm{~g}, 14.1 \mathrm{mmol})$, stirred at $100^{\circ} \mathrm{C}$ for $6 \mathrm{~h}$ and concentrated under reduced pressure. The resulting residue in $\mathrm{MeOH}$ $(50 \mathrm{ml})$ was added to $1 \mathrm{~N} \mathrm{HCl}(100 \mathrm{ml})$ and stirred at room temperature for $20 \mathrm{~min}$. The mixture was added to the saturated aqueous $\mathrm{NaHCO}_{3}$, then extracted with ethyl acetate, dried over $\mathrm{Na}_{2} \mathrm{SO}_{4}$, filtrated and concentrated under reduced pressure. The resulting residue was purified by silica gel column chromatography $\left(\mathrm{CHCl}_{3} / \mathrm{MeOH} / 28 \%\right.$ aq. $\left.\mathrm{NH}_{4} \mathrm{OH}=20 / 1 / 0.1\right)$ to obtain the title compound as a colorless solid $(1.25 \mathrm{~g}, 27.6 \%$ in three steps from 23$)$. ESI-MS $(m / z) 643(\mathrm{M}+\mathrm{H})^{+}$as $\mathrm{C}_{30} \mathrm{H}_{46} \mathrm{~N}_{2} \mathrm{O}_{9} \mathrm{~S}_{2} ;{ }^{1} \mathrm{H}$ NMR $\left(400 \mathrm{MHz}, \mathrm{CD}_{3} \mathrm{OD}\right)$ $\delta$ 0.86-1.00 (m, 3H), 1.25-1.65 (m, 16H), 1.45, 1.48 ( $\mathrm{s} 2,9 \mathrm{H}), 1.73-1.99$ $(\mathrm{m}, 1 \mathrm{H}), 1.79,1.85(\mathrm{~s} \times 2,3 \mathrm{H}), 2.07-2.20(\mathrm{~m}, 1 \mathrm{H}), 2.22-2.42(\mathrm{~m}, 1 \mathrm{H})$, 2.92-3.01 (m, 1H), 3.53-3.61 (m, 1H), 3.63-3.74 (m, 1H), $3.88(\mathrm{~s}, 3 \mathrm{H})$, $3.90-4.15(\mathrm{~m}, 3 \mathrm{H}), 4.28-4.50(\mathrm{~m}, 2 \mathrm{H}), 4.54-4.68(\mathrm{~m}, 1 \mathrm{H}), 5.24(\mathrm{~d}, J=5.4 \mathrm{~Hz}$, $1 \mathrm{H}), 7.39-7.46(\mathrm{~m}, 2 \mathrm{H})$ and $7.89-7.96(\mathrm{~m}, 2 \mathrm{H})$.

7(S)-1'-N-(tert-butoxycarbonyl)-1'-demethyl-7-deoxy-4'-depropyl-7(4-(methoxycarbonyl)phenyl)thio-4'-\{(E)-pent-2-enyl $\}$ lincomycin (61) Compound $27(1.45 \mathrm{~g}, 1.97 \mathrm{mmol}), \mathrm{Et}_{3} \mathrm{~N}(0.69 \mathrm{ml}, 4.93 \mathrm{mmol})$ and methanesulfonyl chloride $(0.31 \mathrm{ml}, 3.93 \mathrm{mmol})$ in $\mathrm{CHCl}_{3}(6.1 \mathrm{ml})$ were treated at room temperature for $30 \mathrm{~min}$ according to the similar procedure as described for the preparation of 60 to afford $1^{\prime}-N$-(tert-butoxycarbonyl)-1'-demethyl-4'-depropyl-7-O-methanesulfonyl-4' $\{(E)$-pent-2-enyl $\}-2,3,4$-tris-O-(trimethylsilyl)lincomycin. To a solution of crude compound were added $\mathrm{K}_{2} \mathrm{CO}_{3}(1.23 \mathrm{~g}$, $8.87 \mathrm{mmol})$ and methyl 4-mercaptobenzoate $(0.99 \mathrm{~g}, 5.91 \mathrm{mmol})$ in DMF $(13.8 \mathrm{ml})$ and treated at $80^{\circ} \mathrm{C}$ for $1 \mathrm{~h}$. The resulting residue and $1 \mathrm{~N} \mathrm{HCl}$ $(14 \mathrm{ml})$ in $\mathrm{MeOH}(14 \mathrm{ml})$ were treated at room temperature for $20 \mathrm{~min}$ according to the similar procedure as described for the preparation of $\mathbf{6 0}$ to afford 61 (1.21 g, 92\% in three steps from 27) as a colorless solid. EI-MS $\mathrm{m} / \mathrm{z}$ $668(\mathrm{M})^{+}$as $\mathrm{C}_{32} \mathrm{H}_{48} \mathrm{~N}_{2} \mathrm{O}_{9} \mathrm{~S}_{2} ;{ }^{1} \mathrm{H}$ NMR $\left(400 \mathrm{MHz}, \mathrm{CD}_{3} \mathrm{OD}\right) \delta 0.95(\mathrm{t}, J=7.4 \mathrm{~Hz}$, $3 \mathrm{H}), 1.33-1.44(\mathrm{~m}, 3 \mathrm{H}), 1.45,1.48$ ( $\mathrm{x} 2,9 \mathrm{H}), 1.80,1.85$ ( $\mathrm{s} 2,3 \mathrm{H}), 1.87-2.17$ $(\mathrm{m}, 6 \mathrm{H}), 2.26-2.40(\mathrm{~m}, 1 \mathrm{H}), 3.01-3.09(\mathrm{~m}, 1 \mathrm{H}), 3.51-3.67(\mathrm{~m}, 2 \mathrm{H}), 3.88$ (s, 3H), 3.91-4.12 (m, 3H), 4.27-4.46 (m, 2H), 4.53-4.64 (m, $1 \mathrm{H}), 5.24$ $(\mathrm{d}, J=5.6 \mathrm{~Hz}, 1 \mathrm{H}), 5.31-5.45(\mathrm{~m}, 1 \mathrm{H}), 5.47-5.59(\mathrm{~m}, 1 \mathrm{H}), 7.36-7.44(\mathrm{~m}, 2 \mathrm{H})$ and $7.86-7.95(\mathrm{~m}, 2 \mathrm{H})$.

$7(S)-1^{\prime}-N$-(tert-butoxycarbonyl)-1'-demethyl-7-deoxy-4'-depropyl7-\{4-(methoxycarbonyl)phenylthio $\}-4^{\prime}-n$-pentyllincomycin (62) Compound $61(105 \mathrm{mg}, 0.16 \mathrm{mmol})$ and $\mathrm{Pd} / \mathrm{C}(100 \mathrm{mg})$ in $\mathrm{MeOH}(2 \mathrm{ml})$ were treated for $15 \mathrm{~h}$ according to the similar procedure as described for the preparation of 7 to afford $\mathbf{6 2}(97 \mathrm{mg}, \mathbf{9 2 . 1 \%})$ as a colorless solid. ESI-MS $\mathrm{m} / \mathrm{z}$ $671(\mathrm{M}+\mathrm{H})^{+}$as $\mathrm{C}_{32} \mathrm{H}_{50} \mathrm{~N}_{2} \mathrm{O}_{9} \mathrm{~S}_{2} ;{ }^{1} \mathrm{H}$ NMR $\left(400 \mathrm{MHz}, \mathrm{CD}_{3} \mathrm{OD}\right) \delta 0.18-0.92$ $(\mathrm{m}, 3 \mathrm{H}), 1.15-1.52(\mathrm{~m}, 11 \mathrm{H}), 1.44,1.47(\mathrm{~s} \times 2,9 \mathrm{H}), 1.71-1.93(\mathrm{~m}, 1 \mathrm{H}), 1.78$, $1.83(\mathrm{~s} \times 2,3 \mathrm{H}), 2.05-2.18(\mathrm{~m}, 1 \mathrm{H}), 2.20-2.36(\mathrm{~m}, 1 \mathrm{H}), 2.95(\mathrm{br} \mathrm{t}, J=10.0 \mathrm{~Hz}$, $1 \mathrm{H}), 3.52-3.61(\mathrm{~m}, 1 \mathrm{H}), 3.61-3.73(\mathrm{~m}, 1 \mathrm{H}), 3.87(\mathrm{~s}, 3 \mathrm{H}), 3.91-4.03(\mathrm{~m}, 2 \mathrm{H})$, $4.03-4.15(\mathrm{~m}, 1 \mathrm{H}), 4.28-4.47(\mathrm{~m}, 2 \mathrm{H}), 4.53-4.65(\mathrm{~m}, 1 \mathrm{H}), 5.25(\mathrm{~d}, J=5.6 \mathrm{~Hz}$, $1 \mathrm{H}), 7.35-7.47(\mathrm{~m}, 2 \mathrm{H})$ and $7.85-7.95(\mathrm{~m}, 2 \mathrm{H})$. 
$7(S)-1^{\prime}-N$-(tert-butoxycarbonyl)-7-\{4-(carboxyl)phenylthio $\}-1^{\prime}-$ demethyl-7-deoxylincomycin (63)

To a solution of compound $\mathbf{6 0}(761 \mathrm{mg}, 1.18 \mathrm{mmol})$ in $\mathrm{MeOH}(20 \mathrm{ml})$ was added $1 \mathrm{~N} \mathrm{NaOH}(1.78 \mathrm{ml}, 1.78 \mathrm{mmol})$ and stirred at room temperature for 7 days. The mixture was added $1 \mathrm{~N} \mathrm{HCl}(\mathrm{pH}=3)$, extracted with $\mathrm{CHCl}_{3}$, dried over $\mathrm{Na}_{2} \mathrm{SO}_{4}$ and concentrated under reduced pressure to obtain the title compound (705 mg, 94.7\%) as an off-white solid. ESI-MS $m / z 629(\mathrm{M}+\mathrm{H})^{+}$as $\mathrm{C}_{29} \mathrm{H}_{44} \mathrm{~N}_{2} \mathrm{O}_{9} \mathrm{~S}_{2}$.

$7(S)-1^{\prime}-N-$ (tert-butoxycarbonyl)-7-\{4-(carboxyl)phenylthio $\}-1^{\prime}-$ demethyl-7-deoxy-4'-depropyl-4' $-n$-pentyllincomycin (64)

Compound $62(97 \mathrm{mg}, 0.15 \mathrm{mmol})$ and $1 \mathrm{~N} \mathrm{NaOH}(0.55 \mathrm{ml})$ in $\mathrm{MeOH}$ $(1.1 \mathrm{ml})$ were treated for $18 \mathrm{~h}$ according to the similar procedure as described for the preparation of $\mathbf{6 3}$ to afford $\mathbf{6 4}(91.3 \mathrm{mg}, 96.1 \%)$ as a colorless solid. FAB-MS $m / z 695(\mathrm{M}+\mathrm{K})^{+}$as $\mathrm{C}_{31} \mathrm{H}_{48} \mathrm{~N}_{2} \mathrm{O}_{9} \mathrm{~S}_{2} ;{ }^{1} \mathrm{H}$ NMR $\left(400 \mathrm{MHz}, \mathrm{CD}_{3} \mathrm{OD}\right)$ $\delta$ 0.82-0.92 (m, 3H), 1.20-1.45 (m, 11H), 1.46, 1.48 (s x 2, 9H), 1.75-1.95 $(\mathrm{m}, 1 \mathrm{H}), 1.81,1.86(\mathrm{~s} \times 2,3 \mathrm{H}), 2.09-2.19(\mathrm{~m}, 1 \mathrm{H}), 2.21-2.37(\mathrm{~m}, 1 \mathrm{H})$, 2.90-3.02 (m, 1H), 3.54-3.62 (m, 1H), 3.63-3.75 (m, 1H), 3.90-4.05 (m, $2 \mathrm{H})$, $4.05-4.15(\mathrm{~m}, 1 \mathrm{H}), 4.30-4.49(\mathrm{~m}, 2 \mathrm{H}), 4.53-4.67(\mathrm{~m}, 1 \mathrm{H}), 5.27(\mathrm{~d}, J=5.6 \mathrm{~Hz}$, $1 \mathrm{H}), 7.37-7.45(\mathrm{~m}, 2 \mathrm{H})$ and $7.89-7.97(\mathrm{~m}, 2 \mathrm{H})$.

\section{7(S)-1'-N-(tert-butoxycarbonyl)-1'-demethyl-7-deoxy-7-\{4-} (morpholinocarbonyl)phenylthio\}lincomycin (65)

To a solution of compound 63 (200 mg, $0.32 \mathrm{mmol})$, 1-hydroxybenzotriazole (64.5 $\mathrm{mg}, 0.48 \mathrm{mmol}$ ) and 1-ethyl-3-(3-dimethylaminopropyl)carbodiimide $\mathrm{HCl}$ salt $(91.5 \mathrm{mg}, 0.48 \mathrm{mmol})$ in DMF $(2 \mathrm{ml})$ was added to morpholine $(42 \mu \mathrm{l}, 0.48 \mathrm{mmol})$ and stirred at room temperature for $22 \mathrm{~h}$. The mixture was added to saturated aqueous $\mathrm{NaHCO}_{3}$, extracted with ethyl acetate, dried over $\mathrm{Na}_{2} \mathrm{SO}_{4}$ and concentrated under reduced pressure. The resulting residue was purified by preparative TLC $\left(\mathrm{CHCl}_{3} / \mathrm{MeOH} / 28 \%\right.$ aq. $\left.\mathrm{NH}_{4} \mathrm{OH}=9 / 2 / 0.2\right)$ to obtain the title compound (215.0 mg, 96.8\%) as a colorless solid. ESI-MS $\mathrm{m} / \mathrm{z}$ $698(\mathrm{M}+\mathrm{H})^{+}$as $\mathrm{C}_{33} \mathrm{H}_{51} \mathrm{~N}_{3} \mathrm{O}_{9} \mathrm{~S}_{2} ;{ }^{1} \mathrm{H}$ NMR $\left(400 \mathrm{MHz}, \mathrm{CD}_{3} \mathrm{OD}\right) \delta 0.86-0.97$ $(\mathrm{m}, 3 \mathrm{H}), 1.25-1.45(\mathrm{~m}, 7 \mathrm{H}), 1.46,1.48(\mathrm{~s} \times 2,9 \mathrm{H}), 1.78-1.95(\mathrm{~m}, 1 \mathrm{H}), 1.88$, $1.91(\mathrm{~s} \times 2,3 \mathrm{H}), 2.06-2.18(\mathrm{~m}, 1 \mathrm{H}), 2.24-2.40(\mathrm{~m}, 1 \mathrm{H}), 2.92-2.99(\mathrm{~m}, 1 \mathrm{H})$, $3.37-3.85(\mathrm{~m}, 10 \mathrm{H}), 3.88-3.99(\mathrm{~m}, 2 \mathrm{H}), 4.05-4.14(\mathrm{~m}, 1 \mathrm{H}), 4.30-4.45(\mathrm{~m}, 2 \mathrm{H})$, $4.52-4.63(\mathrm{~m}, 1 \mathrm{H}), 5.26(\mathrm{~d}, J=5.6 \mathrm{~Hz}, 1 \mathrm{H}), 7.35-7.42(\mathrm{~m}, 2 \mathrm{H})$ and $7.43-7.48$ (m, 2H).

\section{$7(S)-1^{\prime}$-N-(tert-butoxycarbonyl)-1'-demethyl-7-deoxy-4'-depropyl-} 7-\{4-(morpholinocarbonyl)phenylthio $\}-4^{\prime}-n$-pentyllincomycin (66) Compound $64(91.3 \mathrm{mg}, \quad 0.14 \mathrm{mmol}), \quad$ 1-hydroxybenzotriazole $(28.1 \mathrm{mg}$, $0.21 \mathrm{mmol}$ ) and 1-ethyl-3-(3-dimethylaminopropyl)carbodiimide $\mathrm{HCl}$ salt $(40.0 \mathrm{mg}, 0.21 \mathrm{mmol})$ and morpholine $(18 \mu \mathrm{l}, 0.21 \mathrm{mmol})$ in DMF $(1 \mathrm{ml})$ were treated for $62 \mathrm{~h}$ according to the similar procedure as described for the preparation of $\mathbf{6 5}$ to afford $\mathbf{6 6}(82.0 \mathrm{mg}, 81.3 \%)$ as a colorless solid. FAB-MS $m / z 726(\mathrm{M}+\mathrm{H})^{+}$as $\mathrm{C}_{35} \mathrm{H}_{55} \mathrm{~N}_{3} \mathrm{O}_{9} \mathrm{~S}_{2} ;{ }^{1} \mathrm{H}$ NMR $\left(400 \mathrm{MHz}, \mathrm{CD}_{3} \mathrm{OD}\right) \delta 0.82-0.94$ (m, 3H), 1.21-1.42 (m, 11H), 1.46, 1.48 (s x 2, 9H), 1.77-1.95 (m, 1H), 1.87, $1.91(\mathrm{~s} \times 2,3 \mathrm{H}), 2.08-2.18(\mathrm{~m}, 1 \mathrm{H}), 2.20-2.38(\mathrm{~m}, 1 \mathrm{H}), 2.90-3.01(\mathrm{~m}, 1 \mathrm{H})$, $3.38-3.84(\mathrm{~m}, 10 \mathrm{H}), 3.87-4.01(\mathrm{~m}, 2 \mathrm{H}), 4.05-4.14(\mathrm{~m}, 1 \mathrm{H}), 4.29-4.46(\mathrm{~m}, 2 \mathrm{H})$, $4.52-4.63(\mathrm{~m}, 1 \mathrm{H}), 5.26(\mathrm{~d}, J=5.6 \mathrm{~Hz}, 1 \mathrm{H}), 7.35-7.42(\mathrm{~m}, 2 \mathrm{H})$ and $7.42-7.48$ $(\mathrm{m}, 2 \mathrm{H})$.

\section{7(S)-1'-demethyl-7-deoxy-7-\{4-(morpholinocarbonyl)phenylthio $\}$ lincomycin $(67)$}

To the compound $\mathbf{6 5}(215 \mathrm{mg}, 0.32 \mathrm{mmol})$ was added 2,2,2-trifluoroacetic acid $(2 \mathrm{ml})$ at $0{ }^{\circ} \mathrm{C}$ and stirred for $40 \mathrm{~min}$. The solution was concentrated under reduced pressure. The resulting residue was purified by preparative TLC $\left(\mathrm{CHCl}_{3} / \mathrm{MeOH} / 28 \%\right.$ aq. $\left.\mathrm{NH}_{4} \mathrm{OH}=9 / 2 / 0.2\right)$ to obtain the title compound $(104.0 \mathrm{mg}, 56.5 \%)$ as a colorless solid. $[\alpha]_{\mathrm{D}}^{25}+70.5^{\circ}(c 0.42$, MeOH); ESIMS $m / z 598(\mathrm{M}+\mathrm{H})^{+}$as $\mathrm{C}_{28} \mathrm{H}_{43} \mathrm{~N}_{3} \mathrm{O}_{7} \mathrm{~S}_{2}$; TOF-ESI-HRMS $(\mathrm{M}+\mathrm{H})^{+}$calcd. for $\mathrm{C}_{28} \mathrm{H}_{43} \mathrm{~N}_{3} \mathrm{O}_{7} \mathrm{~S}_{2}$ : 598.2621, found: 598.2623; ${ }^{1} \mathrm{H}$ NMR (400 MHz, $\left.\mathrm{CD}_{3} \mathrm{OD}\right)$ $\delta 0.88-0.97(\mathrm{~m}, 3 \mathrm{H}), 1.30-1.44(\mathrm{~m}, 4 \mathrm{H}), 1.33(\mathrm{~d}, J=7.0 \mathrm{~Hz}, 3 \mathrm{H}), 1.80-1.93$ $(\mathrm{m}, 1 \mathrm{H}), 1.90(\mathrm{~s}, 3 \mathrm{H}), 2.05-2.18(\mathrm{~m}, 2 \mathrm{H}), 2.65(\mathrm{dd}, J=10.4,8.2 \mathrm{~Hz}, 1 \mathrm{H})$, $3.26-3.32(\mathrm{~m}, 1 \mathrm{H}), 3.35-3.56(\mathrm{~m}, 2 \mathrm{H}), 3.56(\mathrm{dd}, J=10.2,3.3 \mathrm{~Hz}, 1 \mathrm{H})$, $3.52-3.85$ (m, 6H), 3.79 (br dd, $J=3.3,0.8 \mathrm{~Hz}, 1$ ), 3.90-3.98 (m, 2H), 4.08 (dd, $J=10.2,5.6 \mathrm{~Hz}, 1 \mathrm{H}), 4.37$ (br dd, $J=9.9,0.8 \mathrm{~Hz}, 1 \mathrm{H}), 4.54$ (dd, $J=9.9$, $2.7 \mathrm{~Hz}, 1 \mathrm{H}), 5.25(\mathrm{~d}, J=5.6 \mathrm{~Hz}, 1 \mathrm{H}), 7.35-7.41(\mathrm{~m}, 2 \mathrm{H})$ and $7.43-7.49$ $(\mathrm{m}, 2 \mathrm{H})$.

7(S)-1'-demethyl-7-deoxy-4'-depropyl-7-\{4-(morpholinocarbonyl) phenylthio $\}-4^{\prime}-n$-pentyllincomycin (68)

Compound 66 ( $82.0 \mathrm{mg}, 0.11 \mathrm{mmol})$ and 2,2,2-trifluoroacetic acid $(1 \mathrm{ml})$ were treated at -15 to $0{ }^{\circ} \mathrm{C}$ for $40 \mathrm{~min}$ according to the similar procedure as described for the preparation of 67 to afford $\mathbf{6 8}(57.0 \mathrm{mg}, 80.6 \%)$ as a colorless

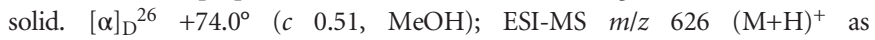
$\mathrm{C}_{30} \mathrm{H}_{47} \mathrm{~N}_{3} \mathrm{O}_{7} \mathrm{~S}_{2}$; TOF-ESI-HRMS $(\mathrm{M}+\mathrm{H})^{+}$calcd. for $\mathrm{C}_{30} \mathrm{H}_{47} \mathrm{~N}_{3} \mathrm{O}_{7} \mathrm{~S}_{2}$ : 626.2934, found: 626.2924; ${ }^{1} \mathrm{H}$ NMR (400 MHz, $\left.\mathrm{CD}_{3} \mathrm{OD}\right) \delta 0.83-0.93(\mathrm{~m}, 3 \mathrm{H}), 1.23-1.44$ $(\mathrm{m}, 8 \mathrm{H}), 1.33(\mathrm{~d}, J=6.9 \mathrm{~Hz}, 3 \mathrm{H}), 1.77-1.88(\mathrm{~m}, 1 \mathrm{H}), 1.90(\mathrm{~s}, 3 \mathrm{H}), 1.99-2.15$ $(\mathrm{m}, 2 \mathrm{H}), 2.61(\mathrm{dd}, J=10.4,8.2 \mathrm{~Hz}, 1 \mathrm{H}), 3.24(\mathrm{dd}, J=10.4,7.0 \mathrm{~Hz}, 1 \mathrm{H})$, 3.36-3.57 (m, 2H), $3.56(\mathrm{dd}, J=10.2,3.2 \mathrm{~Hz}, 1 \mathrm{H}), 3.57-3.84(\mathrm{~m}, 6 \mathrm{H}), 3.78$ (br dd, $J=3.2,0.7 \mathrm{~Hz}, 1 \mathrm{H}$ ), 3.88 (dd, $J=9.5,4.0 \mathrm{~Hz}, 1 \mathrm{H}$ ), 3.95 (dq, $J=6.9$, $2.6 \mathrm{~Hz}, 1 \mathrm{H}), 4.08(\mathrm{dd}, J=10.2,5.6 \mathrm{~Hz}, 1 \mathrm{H}), 4.36(\mathrm{br} \mathrm{dd}, J=9.9,0.7 \mathrm{~Hz}, 1 \mathrm{H})$, $4.53(\mathrm{dd}, J=9.9,2.6 \mathrm{~Hz}, 1 \mathrm{H}), 5.25(\mathrm{~d}, J=5.6 \mathrm{~Hz}, 1 \mathrm{H}), 7.34-7.41(\mathrm{~m}, 2 \mathrm{H})$ and $7.42-7.50(\mathrm{~m}, 2 \mathrm{H})$.

7(S)-7-deoxy-4'-depropyl-7-\{4-(morpholinocarbonyl)phenylthio\}$4^{\prime}$-n-pentyllincomycin (69)

Compound $68(31.0 \mathrm{mg}, 0.050 \mathrm{mmol}), 36 \%$ aqueous formaldehyde $(12 \mu \mathrm{l}$, $0.15 \mathrm{mmol}), \mathrm{AcOH} \quad(17 \mu \mathrm{l}, 0.30 \mathrm{mmol})$ and $\mathrm{NaBH}(\mathrm{OAc})_{3} \quad(31.6 \mathrm{mg}$, $0.15 \mathrm{mmol})$ in $\mathrm{MeOH}(0.5 \mathrm{ml})$ were treated at room temperature for $30 \mathrm{~min}$ according to the similar procedure as described for the preparation of $\mathbf{3 8}$ to afford $69(31.0 \mathrm{mg}, 97.8 \%)$ as a colorless solid. $[\alpha]_{\mathrm{D}}{ }^{26}+68^{\circ}(c 0.12, \mathrm{MeOH})$; ESI-MS $m / z 640(\mathrm{M}+\mathrm{H})^{+}$as $\mathrm{C}_{31} \mathrm{H}_{49} \mathrm{~N}_{3} \mathrm{O}_{7} \mathrm{~S}_{2}$; TOF-ESI-HRMS $(\mathrm{M}+\mathrm{H})^{+}$calcd. for $\mathrm{C}_{31} \mathrm{H}_{49} \mathrm{~N}_{3} \mathrm{O}_{7} \mathrm{~S}_{2}$ : 640.3090, found: 640.3080; ${ }^{1} \mathrm{H}$ NMR (400 MHz, $\mathrm{CD}_{3} \mathrm{OD}$ ) $\delta$ 0.85-0.94 (m, 3H), 1.23-1.43 (m, 8H), $1.35(\mathrm{~d}, J=6.8 \mathrm{~Hz}, 3 \mathrm{H}), 1.81-1.92$ (m, $1 \mathrm{H}), 1.91(\mathrm{~s}, 3 \mathrm{H}), 2.03$ (ddd, $J=13.0,7.8,5.0 \mathrm{~Hz}, 1 \mathrm{H}), 2.08-2.25(\mathrm{~m}, 2 \mathrm{H})$, $2.44(\mathrm{~s}, 3 \mathrm{H}), 3.06(\mathrm{dd}, J=10.5,4.9 \mathrm{~Hz}, 1 \mathrm{H}), 3.25-3.30(\mathrm{~m}, 1 \mathrm{H}), 3.38-3.84$ $(\mathrm{m}, 9 \mathrm{H}), 3.58(\mathrm{dd}, J=10.2,3.2 \mathrm{~Hz}, 1 \mathrm{H}), 3.97(\mathrm{dq}, J=6.8,2.6 \mathrm{~Hz}, 1 \mathrm{H}), 4.10$ (dd, $J=10.2,5.6 \mathrm{~Hz}, 1 \mathrm{H}), 4.33-4.39(\mathrm{~m}, 1 \mathrm{H}), 4.51(\mathrm{dd}, J=9.7,2.6 \mathrm{~Hz}, 1 \mathrm{H})$, $5.26(\mathrm{~d}, J=5.6 \mathrm{~Hz}, 1 \mathrm{H}), 7.37-7.42(\mathrm{~m}, 2 \mathrm{H})$ and $7.45-7.50(\mathrm{~m}, 2 \mathrm{H})$.

\section{7(S)-7-acetylthio-1'-N-(tert-butoxycarbonyl)-1'-demethyl-7-} deoxylincomycin (70)

To a solution of compound 23 (500.3 mg, $0.71 \mathrm{mmol})$ in $\mathrm{CH}_{2} \mathrm{Cl}_{2}(10 \mathrm{ml})$ were added $\mathrm{Et}_{3} \mathrm{~N}(0.25 \mathrm{ml}, 1.77 \mathrm{mmol})$ and methanesulfonyl chloride $(0.11 \mathrm{ml}$, $1.39 \mathrm{mmol}$ ), stirred at $0{ }^{\circ} \mathrm{C}$ for $1 \mathrm{~h}$. The mixture was added to saturated aqueous $\mathrm{NH}_{4} \mathrm{Cl}$, extracted with ethyl acetate, washed with $25 \%$ brine, dried over $\mathrm{Na}_{2} \mathrm{SO}_{4}$ and concentrated under reduced pressure. To a solution of this crude compound in DMF ( $8 \mathrm{ml}$ ) was added AcSK $(501.7 \mathrm{mg}, 4.39 \mathrm{mmol})$, stirred at $60^{\circ} \mathrm{C}$ for $10 \mathrm{~h}$. The mixture was added to the saturated aqueous $\mathrm{NaHCO}_{3}$, then extracted with ethyl acetate, dried over $\mathrm{Na}_{2} \mathrm{SO}_{4}$, filtrated and concentrated under reduced pressure. The resulting residue was purified by silica gel column chromatography (hexane/ethyl acetate $=10 / 1$ to $2 / 1$ ) to obtain 7(S)-7-acetylthio-1'-N-(tert-butoxycarbonyl)-1'-demethyl-7-deoxy-2,3,4tris- $O$-(trimethylsilyl)lincomycin as a colorless solid $(217.8 \mathrm{mg}, 40.2 \%$ in two steps from 23). To a solution of this intermediate in $\mathrm{MeOH}(2.2 \mathrm{ml})$ was added $1 \mathrm{~N} \mathrm{HCl}(2.2 \mathrm{ml})$ and stirred at room temperature for $1 \mathrm{~h}$. The mixture was added to saturated aqueous $\mathrm{NaHCO}_{3}$, extracted with ethyl acetate, dried over $\mathrm{Na}_{2} \mathrm{SO}_{4}$ and concentrated under reduced pressure. The resulting residue was purified by silica gel column chromatography (chloroform/ $\mathrm{MeOH}=40 / 1)$ to obtain the title compound (137 mg, 87.6\%) as a colorless solid. ESI-MS $\mathrm{m} / \mathrm{z} 551$ $(\mathrm{M}+\mathrm{H})^{+}$as $\mathrm{C}_{24} \mathrm{H}_{42} \mathrm{~N}_{2} \mathrm{O}_{8} \mathrm{~S}_{2} ;{ }^{1} \mathrm{H} \quad \mathrm{NMR}\left(400 \mathrm{MHz}, \mathrm{CD}_{3} \mathrm{OD}\right) \quad \delta \quad 0.86-0.98$ $(\mathrm{m}, 3 \mathrm{H}), 1.26-1.41(\mathrm{~m}, 7 \mathrm{H}), 1.46(\mathrm{~s}, 9 \mathrm{H}), 1.76-1.96(\mathrm{~m}, 1 \mathrm{H}), 2.01,2.03$ (s, 3H), 1.95-2.17 (m, 1H), 2.20-2.38 (m, 1H), $2.32(\mathrm{~s}, 3 \mathrm{H}), 2.95(\mathrm{t}, J=9.8 \mathrm{~Hz}$, $1 \mathrm{H}), 3.51(\mathrm{dd}, J=10.2,3.3 \mathrm{~Hz}, 1 \mathrm{H}), 3.66(\mathrm{dd}, J=10.0,7.6 \mathrm{~Hz}, 1 \mathrm{H}), 3.81-4.01$ $(\mathrm{m}, 2 \mathrm{H}), 4.01-4.12(\mathrm{~m}, 1 \mathrm{H}), 4.13-4.37(\mathrm{~m}, 2 \mathrm{H}), 4.41-4.50(\mathrm{~m}, 1 \mathrm{H})$ and 5.21 $(\mathrm{d}, J=5.5 \mathrm{~Hz}, 1 \mathrm{H})$. 
7(S)-1'-N-(tert-butoxycarbonyl)-1'-demethyl-7-deoxy-7mercaptolincomycin (71)

To a solution of compound $70(137 \mathrm{mg}, 0.25 \mathrm{mmol})$ in $\mathrm{MeOH}(1.5 \mathrm{ml})$ was added sodium methoxide $(43.2 \mathrm{mg}, 0.76 \mathrm{mmol}$ ) and stirred at room temperature for $1.5 \mathrm{~h}$. The mixture was diluted with $8 \%$ aqueous $\mathrm{NaHCO}_{3}$, extracted with ethyl acetate, dried over $\mathrm{Na}_{2} \mathrm{SO}_{4}$ and concentrated under reduced pressure. The resulting residue was purified by silica gel column chromatography $\left(\mathrm{CHCl}_{3} / \mathrm{CH}_{3} \mathrm{OH}=40 / 1\right.$ to $\left.10 / 1\right)$ to obtain the title compound $(120.3 \mathrm{mg}$, 95.1\%) as a colorless solid. FAB-MS $m / z 509(\mathrm{M}+\mathrm{H})^{+}$as $\mathrm{C}_{22} \mathrm{H}_{40} \mathrm{~N}_{2} \mathrm{O}_{7} \mathrm{~S}_{2} ;{ }^{1} \mathrm{H}$ NMR $\left(400 \mathrm{MHz}, \mathrm{CD}_{3} \mathrm{OD}\right) \delta 0.87-0.99(\mathrm{~m}, 3 \mathrm{H}), 1.23-1.42(\mathrm{~m}, 7 \mathrm{H}), 1.47$ (s, 9H), 1.80-1.98 (m, 1H), 2.05-2.15 (m, 1H), $2.15(\mathrm{~s}, 3 \mathrm{H}), 2.20-2.39$ (m, 1H), 2.97 (br t, $J=9.6 \mathrm{~Hz}, 1 \mathrm{H}), 3.39-3.58(\mathrm{~m}, 1 \mathrm{H}), 3.54(\mathrm{dd}, J=10.2$, $3.0 \mathrm{~Hz}, 1 \mathrm{H}), 3.66(\mathrm{dd}, J=10.2,7.4 \mathrm{~Hz}, 1 \mathrm{H}), 3.80-3.90(\mathrm{~m}, 1 \mathrm{H}), 4.02-4.18$ $(\mathrm{m}, 2 \mathrm{H}), 4.26-4.46(\mathrm{~m}, 2 \mathrm{H})$ and $5.25(\mathrm{~d}, J=5.5 \mathrm{~Hz}, 1 \mathrm{H})$.

\section{7(S)-1'-N-(tert-butoxycarbonyl)-1'-demethyl-7-deoxy-7-\{(4- (pyridin-3-yl)phenylthio\}lincomycin (72)}

To a solution of 3-(4-bromophenyl)pyridine $(75.4 \mathrm{mg}, 0.32 \mathrm{mmol}), 4,5$-bis (diphenylphosphino)-9,9-dimethylxanthene $(13.4 \mathrm{mg}, 0.023 \mathrm{mmol}$ ) and tris (dibenzylideneacetone)dipalladium $(0) \quad\left(\mathrm{Pd}_{2}\left(\mathrm{dba}_{3}\right) \quad(12.3 \mathrm{mg}, 13.4 \mu \mathrm{mol})\right.$ in 1,4-dioxane $(1 \mathrm{ml})$ were added to compound $71(120.3 \mathrm{mg}, 0.24 \mathrm{mmol})$ and $\mathrm{N}, \mathrm{N}$-diisopropylethylamine $(82 \mu \mathrm{l}, 0.47 \mathrm{mmol})$ and refluxed for $5 \mathrm{~h}$. The mixture was filtrated by either Chromatodisc $(0.45 \mu \mathrm{m})$ (Kurabo Industries) or celite, concentrated under reduced pressure. The resulting residue was purified by silica gel column chromatography $\left(\mathrm{CHCl}_{3} / \mathrm{MeOH} / 28 \%\right.$ aq. $\left.\mathrm{NH}_{4} \mathrm{OH}=10 / 1 / 0.1\right)$ to obtain the title compound as an off-white solid (125.9 mg, 80.4\%). FAB-MS m/z $662(\mathrm{M}+\mathrm{H})^{+}$as $\mathrm{C}_{33} \mathrm{H}_{47} \mathrm{~N}_{3} \mathrm{O}_{7} \mathrm{~S}_{2} ;{ }^{1} \mathrm{H}$ NMR $\left(400 \mathrm{MHz}, \mathrm{CD}_{3} \mathrm{OD}\right) \delta 0.87-1.00(\mathrm{~m}, 3 \mathrm{H}), 1.21-1.43(\mathrm{~m}, 7 \mathrm{H}), 1.48(\mathrm{~s}, 9 \mathrm{H})$, $1.77-1.95(\mathrm{~m}, 1 \mathrm{H}), 1.94,1.97(\mathrm{~s} \times 2,3 \mathrm{H}), 2.05-2.21(\mathrm{~m}, 1 \mathrm{H}), 2.22-2.44$ (m, 1H), $2.97(\mathrm{t}, J=9.6 \mathrm{~Hz}, 1 \mathrm{H}), 3.59(\mathrm{dd}, J=10.0,3.2 \mathrm{~Hz}, 1 \mathrm{H}), 3.68(\mathrm{br} \mathrm{dd}$, $J=9.8,8.1 \mathrm{~Hz}, 1 \mathrm{H}), 3.85-4.01(\mathrm{~m}, 2 \mathrm{H}), 4.05-4.16(\mathrm{~m}, 1 \mathrm{H}), 4.28-4.41(\mathrm{~m}, 1 \mathrm{H})$, $4.41-4.50(\mathrm{~m}, 1 \mathrm{H}), 4.51-4.64(\mathrm{~m}, 1 \mathrm{H}), 5.27(\mathrm{~d}, J=5.5 \mathrm{~Hz}, 1 \mathrm{H}), 7.47-7.57$ $(\mathrm{m}, 3 \mathrm{H}), 7.59-7.69(\mathrm{~m}, 2 \mathrm{H}), 8.05-8.14(\mathrm{~m}, 1 \mathrm{H}), 8.51(\mathrm{dd}, J=4.8,1.5 \mathrm{~Hz}, 1 \mathrm{H})$ and $8.78-8.83(\mathrm{~m}, 1 \mathrm{H})$.

\section{7(S)-1'-demethyl-7-deoxy-7-\{(4-(pyridin-3-yl)phenylthio $\}$ lincomycin (73)}

Compound $72(125.9 \mathrm{mg}, 0.19 \mathrm{mmol})$ and 2,2,2-trifluoroacetic acid $(0.29 \mathrm{ml})$ in $\mathrm{CH}_{2} \mathrm{Cl}_{2}(2.5 \mathrm{ml})$ were treated at $-20^{\circ} \mathrm{C}$ for $10 \mathrm{~min}$, and then treated at $0{ }^{\circ} \mathrm{C}$ for $3 \mathrm{~h}$ according to the similar procedure as described for the preparation of 67 to afford $73(99.1 \mathrm{mg}, 92.7 \%)$ as a colorless solid. $[\alpha]_{\mathrm{D}}{ }^{25}+91.4^{\circ}$ (c 0.74 , $\mathrm{MeOH})$; ESI-MS $m / z 562(\mathrm{M}+\mathrm{H})^{+}$as $\mathrm{C}_{28} \mathrm{H}_{39} \mathrm{~N}_{3} \mathrm{O}_{5} \mathrm{~S}_{2}$; TOF-ESI-HRMS $(\mathrm{M}+\mathrm{H})$ ${ }^{+}$calcd. for $\mathrm{C}_{28} \mathrm{H}_{39} \mathrm{~N}_{3} \mathrm{O}_{5} \mathrm{~S}_{2}$ : 562.2409 , found: 562.2407 ; ${ }^{1} \mathrm{H}$ NMR $(400 \mathrm{MHz}$, $\left.\mathrm{CD}_{3} \mathrm{OD}\right) \delta 0.86-0.97(\mathrm{~m}, 3 \mathrm{H}), 1.30-1.42(\mathrm{~m}, 4 \mathrm{H}), 1.33(\mathrm{~d}, J=6.9 \mathrm{~Hz}, 3 \mathrm{H})$, $1.77-1.87(\mathrm{~m}, 1 \mathrm{H}), 1.97(\mathrm{~s}, 3 \mathrm{H}), 2.02-2.13(\mathrm{~m}, 2 \mathrm{H}), 2.59(\mathrm{dd}, J=10.3,8.0 \mathrm{~Hz}$, $1 \mathrm{H}), 3.22(\mathrm{dd}, J=10.3,7.0 \mathrm{~Hz}, 1 \mathrm{H}), 3.58(\mathrm{dd}, J=10.2,3.3 \mathrm{~Hz}, 1 \mathrm{H}), 3.79(\mathrm{br} \mathrm{dd}$, $J=3.3,0.9 \mathrm{~Hz}, 1 \mathrm{H}), 3.86(\mathrm{dd}, J=9.3,3.7 \mathrm{~Hz}, 1 \mathrm{H}), 3.93(\mathrm{dq}, J=6.9,2.6 \mathrm{~Hz}$, $1 \mathrm{H}), 4.10(\mathrm{dd}, J=10.2,5.6 \mathrm{~Hz}, 1 \mathrm{H}), 4.36-4.42(\mathrm{~m}, 1 \mathrm{H}), 4.51(\mathrm{dd}, J=9.9$, $2.6 \mathrm{~Hz}, 1 \mathrm{H}), 5.27(\mathrm{~d}, J=5.6 \mathrm{~Hz}, 1 \mathrm{H}), 7.51(\mathrm{ddd}, J=8.0,4.9,0.9 \mathrm{~Hz}, 1 \mathrm{H})$, $7.51-7.58(\mathrm{~m}, 2 \mathrm{H}), 7.60-7.68(\mathrm{~m}, 2 \mathrm{H}), 8.07$ (ddd, $J=8.0,2.4,1.6 \mathrm{~Hz}, 1 \mathrm{H}), 8.51$ $(\mathrm{dd}, J=4.9,1.6 \mathrm{~Hz}, 1 \mathrm{H})$ and $8.79(\mathrm{dd}, J=2.4,0.9 \mathrm{~Hz}, 1 \mathrm{H})$.

\section{7(S)-1'-N-(tert-butoxycarbonyl)-1'-demethyl-7-deoxy-7-\{4- (pyrimidin-5-yl)phenylthio\}lincomycin (74)}

Compound $71 \quad(116.4 \mathrm{mg}, \quad 0.23 \mathrm{mmol}), \quad 5$-(4-bromophenyl)pyrimidine (107.3 mg, $\quad 0.46 \mathrm{mmol}), \quad 4,5$-bis(diphenylphosphino)-9,9-dimethylxanthene (14.2 $\mathrm{mg}, 0.024 \mathrm{mmol}), \mathrm{Pd}_{2}(\mathrm{dba})_{3}(11.8 \mathrm{mg}, 0.013 \mathrm{mmol})$ and $N, N$-diisopropylethylamine $(79.5 \mu \mathrm{l}, 0.46 \mathrm{mmol})$ in 1,4-dioxane $(1.5 \mathrm{ml})$ were treated for $6 \mathrm{~h}$ according to the similar procedure as described for the preparation of 72 to afford $74(117.9 \mathrm{mg}, 77.7 \%)$ as a colorless solid. FAB-MS $\mathrm{m} / z 663(\mathrm{M}+\mathrm{H})^{+}$as $\mathrm{C}_{32} \mathrm{H}_{46} \mathrm{~N}_{4} \mathrm{O}_{7} \mathrm{~S}_{2} ;{ }^{1} \mathrm{H}$ NMR $\left(400 \mathrm{MHz}, \mathrm{CD}_{3} \mathrm{OD}\right) \delta$ 0.86-1.00 (m, 3H), 1.25-1.43 $(\mathrm{m}, 7 \mathrm{H}), 1.48,1.47(\mathrm{~s} \times 2,9 \mathrm{H}), 1.80-1.95(\mathrm{~m}, 1 \mathrm{H}), 1.92,1.95(\mathrm{~s} \times 2,3 \mathrm{H})$, 2.04-2.20 (m, $1 \mathrm{H}), 2.21-2.44(\mathrm{~m}, 1 \mathrm{H}), 2.97(\mathrm{t}, J=9.6 \mathrm{~Hz}, 1 \mathrm{H}), 3.56-3.75$ $(\mathrm{m}, 2 \mathrm{H}), 3.88-4.03(\mathrm{~m}, 2 \mathrm{H}), 4.06-4.19(\mathrm{~m}, 1 \mathrm{H}), 4.29-4.42(\mathrm{~m}, 1 \mathrm{H}), 4.46$ (d, $J=9.6 \mathrm{~Hz}, 1 \mathrm{H}), 4.54-4.67(\mathrm{~m}, 1 \mathrm{H}), 5.28(\mathrm{~d}, J=5.5 \mathrm{~Hz}, 1 \mathrm{H}), 7.50-7.59$ (m, 2H), 7.65-7.73 (m, 2H), $9.07(\mathrm{~s}, 2 \mathrm{H}), 9.13(\mathrm{~s}, 1 \mathrm{H})$.

\section{7(S)-1'-demethyl-7-deoxy-7-\{4-(pyrimidin-5-yl)phenylthio $\}$ lincomycin (75)}

Compound 74 (117.9 mg, $0.18 \mathrm{mmol})$ and 2,2,2-trifluoroacetic acid $(0.27 \mathrm{ml})$ in $\mathrm{CH}_{2} \mathrm{Cl}_{2}(2.5 \mathrm{ml})$ were treated at $-20{ }^{\circ} \mathrm{C}$ for $20 \mathrm{~min}$, and then treated room temperature for $5 \mathrm{~h}$ according to the similar procedure as described for the preparation of 73 to afford $75(82.2 \mathrm{mg}, 82.1 \%)$ as a colorless solid. $[\alpha]_{\mathrm{D}}{ }^{25}$ $+91.7^{\circ}\left(c\right.$ 0.33, MeOH); ESI-MS $m / z 563(\mathrm{M}+\mathrm{H})^{+}$as $\mathrm{C}_{27} \mathrm{H}_{38} \mathrm{~N}_{4} \mathrm{O}_{5} \mathrm{~S}_{2}$; TOF-ESIHRMS $(\mathrm{M}+\mathrm{H})^{+}$calcd. for $\mathrm{C}_{27} \mathrm{H}_{38} \mathrm{~N}_{4} \mathrm{O}_{5} \mathrm{~S}_{2}: 563.2362$, found: 563.2356; ${ }^{1} \mathrm{H}$ NMR $\left(400 \mathrm{MHz}, \mathrm{CD}_{3} \mathrm{OD}\right) \delta 0.78-0.88(\mathrm{~m}, 3 \mathrm{H}), 1.20-1.32(\mathrm{~m}, 4 \mathrm{H}), 1.25(\mathrm{~d}$, $J=6.9 \mathrm{~Hz}, 3 \mathrm{H}), 1.68-1.78(\mathrm{~m}, 1 \mathrm{H}), 1.86(\mathrm{~s}, 3 \mathrm{H}), 1.92-2.05(\mathrm{~m}, 2 \mathrm{H}), 2.55$ (dd, $J=10.2,8.0 \mathrm{~Hz}, 1 \mathrm{H}), 3.12(\mathrm{dd}, J=10.2,6.7 \mathrm{~Hz}, 1 \mathrm{H}), 3.48(\mathrm{dd}, J=10.2$, $3.3 \mathrm{~Hz}, 1 \mathrm{H}), 3.69$ (br dd, $J=3.3,0.7 \mathrm{~Hz}, 1 \mathrm{H}), 3.75$ (dd, $J=9.1,3.4 \mathrm{~Hz}, 1 \mathrm{H})$, $3.87(\mathrm{dq}, J=6.9,2.6 \mathrm{~Hz}, 1 \mathrm{H}), 4.01(\mathrm{dd}, J=10.2,5.5 \mathrm{~Hz}, 1 \mathrm{H}), 4.27-4.32$ $(\mathrm{m}, 1 \mathrm{H}), 4.43(\mathrm{dd}, J=9.9,2.6 \mathrm{~Hz}, 1 \mathrm{H}), 5.18(\mathrm{~d}, J=5.5 \mathrm{~Hz}, 1 \mathrm{H}), 7.43-7.50$ (m, 2H), 7.55-7.65 (m, 2H), 8.97 (s, 2H), $9.03(\mathrm{~s}, 1 \mathrm{H})$.

\section{In vitro antibacterial activity}

MIC $\left(\mu \mathrm{g} \mathrm{ml}^{-1}\right.$ ) was determined by the agar dilution method, which was described in Clinical and Laboratory Standards Institute (M07-06 in 2003). Test strains of $S$. pneumoniae and $S$. pyogenes were subjected to seed culture using brain heart infusion agar (Becton Dickinson and Company, Tokyo, Japan) and $5 \%$ defibrinated horse blood. Test strains of $H$. influenzae were subjected to seed culture using sensitivity disk agar-N 'Nissui' (Nissui, Tokyo, Japan), 5\% defibrinated horse blood, $5 \mu \mathrm{g} \mathrm{ml}^{-1} \mathrm{Hemin}$ and $15 \mu \mathrm{g} \mathrm{ml}^{-1}$ nicotinamide adenine dinucleotide. A $5 \mu \mathrm{l}$ portion of cell suspension of the test strains having about $10^{6}$ colony-forming unit per $\mathrm{ml}$ was inoculated into sensitivity disk agar$\mathrm{N}$ 'Nissui' supplemented with $5 \%$ defibrinated horse blood, $5 \mu \mathrm{g} \mathrm{ml}^{-1} \mathrm{Hemin}$ and $15 \mu \mathrm{g} \mathrm{ml}{ }^{-1}$ nicotinamide adenine dinucleotide, and incubated at $37^{\circ} \mathrm{C}$ for 18-22 $\mathrm{h}$. Then, the MIC was measured.

\section{CONFLICT OF INTEREST}

The authors declare no conflict of interest.

\section{ACKNOWLEDGEMENTS}

We thank A Tamura, Dr E Shitara and Dr T Yoshida for encouragement and valuable discussion. We are grateful to Professor Emeritus Dr M Konno for supervision through our in-house drug discovery program in lincomycin field. We also thank M Ishii for direction in intellectual properties, T Miyara, S Miki and $\mathrm{K}$ Kaneda for analytical and synthetic chemistry, Y Takayama and $\mathrm{K}$ Yamada for biological studies, and M Takagi for English manuscript.

1 Reinert, R. R., van der Linden, M. \& Al-Lahham, A. Molecular characterization of the first telithromycin-resistant Streptococcus pneumoniae isolate in Germany. Antimicrob. Agents Chemother. 49, 3520-3522 (2005).

$2 \mathrm{Kim}, \mathrm{S}, \mathrm{H}$. et al. Changing trends in antimicrobial resistance and serotypes of Streptococcus pneumoniae isolates in Asian countries: an Asian Network for Surveillance of Resistant Pathogens (ANSORP) study. Antimicrob. Agents Chemother. 56, 1418-1426 (2012).

3 Ajito, K., Miura, T., Furuuchi, T. \& Tamura, A. Sixteen-membered macrolides: chemical modifications and future applications. Heterocycles 89, 281-352 (2014).

4 Morimoto, S., Takahashi, Y., Watanabe, Y. \& Omura, S. Chemical modification of erythromycins. I. Synthesis and antibacterial activity of 6-0-methylerythromycins A J. Antibiot. 37, 187-189 (1984).

5 Slobodan, D. et al. Erythromycin series. Part 13. Synthesis and structure elucidation of 10-dihydro-10-deoxo-11-methyl-11-azaerythromycin A. J. Chem. Res. Synop. 152-153 (1988)

6 Retsema, J. et al. Spectrum and mode of action of azithromycin (CP-62,993), a new 15-membered-ring macrolide with improved potency against Gram-negative organisms. Antimicrob. Agents Chemother 31, 1939-1947 (1987).

7 Denis, A. et al. Synthesis and antibacterial activity of HMR 3647 a new ketolide highly potent against erythromycin-resistant and susceptible pathogens. Bioorg. Med. Chem. Lett. 9, 3075-3080 (1999).

8 Clay, K. D. et al. Severe hepatotoxicity of telithromycin: three case reports and literature review. Ann. Intern. Med. 144, 415-420 (2006)

9 Ross, D. B. The FDA and the case of ketek. N. Engl. J. Med. 356, 1601-1604 (2007). 
10 Gleason, P. P., Walters, C., Heaton, A. H. \& Schafer, J. A. Telithromycin: the perils of hasty adoption and persistence of off-label prescribing. J. Manag. Care Pharm. 13, 20-25 (2007).

11 Department of Health and Human Services. Telithromycin (marketed as ketek) information. Available at: http://www.fda.gov/drugs/drugsafety/postmarketdrugsafetyinformationforpatientsandproviders/ucm107824.htm (last accessed 26 April 2007).

12 Miura, T. et al. Novel azalides derived from sixteen-membered macrolides. I. Isolation of the mobile dialdehyde and its one-pot macrocyclization with an amine. J. Antibiot. 60, 407-435 (2007)

13 Miura, T. et al. Novel azalides derived from 16-membered macrolides. III. Azalides modified at the C-15 and 4" positions: improved antibacterial activities. Bioorg. Med. Chem. 18, 2735-2747 (2010).

14 Mason, D. J., Dietz, A. \& Deboer, C. Lincomycin, a new antibiotic I. Discovery and biological properties. Antimicrob. Agents Chemother. 1962, 554-559 (1962).

15 Magerlein, B. J. \& Lincomycin, X. The chemical synthesis of lincomycin. Tetrahedron Lett. 1, 33-36 (1970)

16 Howarth, G. B., Szarek, W. A. \& Jones, J. K. N. The synthesis of lincomycin. J. Chem. Soc. (c) 16, 2218-2224 (1970).

17 Perlman, D. Structure-Activity Relationships Among the Semisynthetic Antibiotics 600-651 (Harcourt Brace Jovanovich Publishers and Academic Press, New York, San Francisco, London, 1977).

18 Birkenmeyer, R. D. \& Kagan, F. Lincomycin. XI. Synthesis and structure of clindamycin. A potent antibacterial agent. J. Med. Chem. 13, 616-619 (1970).

19 Shan, P. J., Vakil, N. \& Kabakov, A. Role of intravenous immune globulin in streptococcal toxic shock syndrome and Clostridium difficile infection. Am. J. Health Syst. Pharm. 72, 1013-1019 (2015).

20 Hoeksema, H. in Abstr. Pap. Division of Carbohydrate Chemistry, 149th Meet. Am Chem. Soc. Detroit, MI, 9C (The Upjohn Company, Kalamazoo, MI, 1965).

21 Magerlein, B. J., Birkenmeyer, R. D. \& Kagan, F. Chemical modification of lincomycin. Antimicrob. Agents Chemother. 6, 727-736 (1966).

22 Sinkula, A. A., Morozowich, W., Lewis, C. \& Mackellar, F. A. Synthesis and bioactivity of lincomycin-7-monoesters. J. Pharm. Sci. 58, 1389-1392 (1969).

23 Magerlein, B. J. \& Kagan, F. Lincomycin. IX. 7-Thiol and thioamido analogs of lincomycin. J. Med. Chem. 12, 974-977 (1969).

24 Lewis, J. G. et al. in 43rd Interscience Conference on Antimicrobial Agents and Chemotherapy, Poster F-1388 (Washington, DC, USA, 2004).

25 Bannister, B. Modifications of lincomycin involving the carbohydrate portion. Part III. The 7-0-methyl and 6-de-(1-hydroxyethyl) analogues. J. Chem. Soc. Perkin Trans. I 16, 1676-1682 (1973).

26 Bannister, B. Modifications of lincomycin involving the carbohydrate portion. Part IV. (7S)-7-alkoxy-7-deoxy-analogues. J. Chem. Soc. Perkin Trans. / 3, 360-369 (1974).

27 Bannister, B. \& Mydlow, P. K. The S-alkylation of sulphides by an activated carbohydrate epimine under acidic catalysis: The formation of $\alpha$-acetamido-sulphides. Part 5. The introduction of functionality into the sulphide substituent. J. Chem. Res. (S) 1989, 90-91 (1989).

28 Bannister, B. The S-alkylation of sulphides by an activated carbohydrate epimine under acidic catalysis: the formation of $\alpha$-acetamido-sulphides. Part 4. Reaction with dithioacetals and monothioacetals. J. Chem. Soc. Perkin. Trans. / 1980, 540-552 (1980).

29 Bannister, B. (7S)-7-deoxy-7-substituted-alkylthio-lincomycin. S-Alkylation of sulphides by an activated epimine under acidic catalysis: formation of $\alpha$-acetamido-sulphides. Tetrahedron 40, 1633-1660 (1984).

30 Bannister, B. Derivatives of lincomycin and its analogs and process. US Patent US3915954 A (The Upjohn Company, 1973).
31 Bannister, B. Derivatives of lincomycin and its analogs and process. Canadian Patent CA-971956 A1 (The Upjohn Company, 1972).

32 Sztaricskai, F. et al. Semisynthetic modification of antibiotic lincomycin. J. Antibiot. 49, 941-943 (1996).

33 Umemura, E. et al. Lincomycin derivative and antibacterial agent containing the same as active ingredient. Japanese patent WO/2007/066805 A1 (14 June 2007).

34 Wakiyama, Y. et al. Lincomycin derivatives and antibacterial agents containing the same as the active ingredient. Japanese patent: WO/2008/146917 A1 (4 December 2008).

35 Umemura, E. et al. Lincosamide derivative, and antibacterial agent comprising the same as active. Japanese patent WO/2008/146919 A1 (4 December 2008).

36 Umemura, E. et al. Synthesis of novel lincomycin derivatives and their in vitro antibacterial activities. J. Antibiot. 66, 195-198 (2013).

37 Wakiyama, Y. et al. Synthesis and structure-activity relationships of novel lincomycin derivatives. Part 1 . Newly generated antibacterial activities against Gram-positive bacteria with erm gene by C-7 modification. J. Antibiot. 69, 368-380 (2016)

38 Wakiyama, Y. et al. Synthesis and structure-activity relationships of novel lincomycin derivatives. Part 2. Synthesis of 7(S)-7-deoxy-7-(4-morpholinocarbonylphenylthio) lincomycin and its 3-dimensional analysis with rRNA. J. Antibiot. 69, 428-439 (2016).

39 Kumura, K. et al. Synthesis and antibacterial activity of novel lincomycin derivatives. I. Enhancement of antibacterial activities by introduction of substituted azetidines. J. Antibiot. 69, 440-445 (2016).

40 Wakiyama, Y. et al. Synthesis and structure-activity relationships of novel lincomycin derivatives part 3: discovery of the 4-(pyrimidin-5-yl)phenyl group in synthesis of 7(S)-thiolincomycin analogs. J. Antibiot. 70, 52-64 (2017).

41 Kumura, K. et al. Synthesis and antibacterial activity of novel lincomycin derivatives. II. Exploring (7S)-7-(5-aryl-1,3,4-thiadiazol-2-yl-thio)-7-deoxylincomycin derivatives. J. Antibiot. (e-pub ahead of print 7 December 2016; doi:10.1038/ja.2016.139).

42 Argoudelis, A. D., Coats, J. H., Mason, D. J. \& Sebek, O. K. Microbial transformation of antibiotics. III. Conversion of clindamycin to $1^{\prime}$-demethylclindamycin and clindamycin sulfoxide by Streptomyces species. J. Antibiot. 22, 309-314 (1969).

43 Magerlein, B. J., Birkenmeyer, R. D. \& Kagan, F. Lincomycin. VI. 4'-alkyl analogs of lincomycin. Relationship between structure and antibacterial activity. J. Med. Chem. 10, 355-359 (1967)

44 Magerlein, B. J. Lincomycin. 14. An improved synthesis and resolution of the antimalarial agent, 1'-demethyl-4'-depropyl-4' $(R)$ - and -(S)-pentylclindamycin hydrochloride (U-24,729A). J. Med. Chem. 15, 1255-1259 (1972).

45 Magerlein, B. J. Lincomycin. VII. 4'-depropyl-4'-ethoxylincomycins. J. Med. Chem. 10, 1161-1163 (1967)

46 Lewis, J. G. et al. Novel lincomycin derivatives possessing antimicrobial activity. Japanese patent WO/2006/055070 A2 (26 May 2006).

47 Schlünzen, F. et al. Structural basis for the interaction of antibiotics with the peptidyl transferase centre in eubacteria. Nature 413, 814-821 (2001).

48 Pedregal, C., Ezquerra, J., Escribano, A., Carreńo, M. C. \& Ruano, J. G. Highly chemoselective reduction of $\mathrm{N}$-Boc protected lactams. Tetrahedron Lett. 35 , 2053-2056 (1994).

49 Llinàs-Brunet, M. et al. Highly potent and selective peptide-based inhibitors of the hepatitis $C$ virus serine protease: towards smaller inhibitors. Bioorg. Med. Chem. Lett. $10,2267-2270$ (2000).

50 Itoh, T. \& Mase, T. A general palladium-catalyzed coupling of aryl bromides/triflates and thiols. Org. Lett. 6, 4587-4590 (2004). 